University of Tennessee Health Science Center

UTHSC Digital Commons

\title{
A Virtual Model of the Human Cervical Spine for Physics-based Simulation and Applications
}

Hyung Soo Ahn

University of Tennessee Health Science Center

Follow this and additional works at: https://dc.uthsc.edu/dissertations

Part of the Medical Biotechnology Commons, Musculoskeletal, Neural, and Ocular Physiology Commons, and the Other Medical Sciences Commons

\section{Recommended Citation}

Ahn, Hyung Soo, "A Virtual Model of the Human Cervical Spine for Physics-based Simulation and Applications" (2005). Theses and Dissertations (ETD). Paper 7. http://dx.doi.org/10.21007/ etd.cghs.2005.0008.

This Thesis is brought to you for free and open access by the College of Graduate Health Sciences at UTHSC Digital Commons. It has been accepted for inclusion in Theses and Dissertations (ETD) by an authorized administrator of UTHSC Digital Commons. For more information, please contact jwelch30@uthsc.edu. 


\title{
A Virtual Model of the Human Cervical Spine for Physics-based Simulation and Applications
}

\begin{abstract}
Utilizing recent advances in computer technology, Our Biomechanics Laboratory have made an effort to integrate computer animation and engineering analysis software into biomedical research, specifically towards simulation and animation of in vitro experimentation of the human cervical spine in the virtual world. The objectives of this study were to develop a virtual model of the human cervical spine for physics-based simulation and to apply the virtual model to studies of different surgical procedures and instrumentation.

A process for creating an accurate virtual model of the human cervical spine was developed. The model consisted of seven vertebrae (C2-T1) connected with soft tissue components: intervertebral joint, facet joints, and ligaments. The soft tissue components were assigned nonlinear viscoelastic properties. The evaluation of the model included the percent contribution of rotation relative to global rotation, coupling behaviors, helical axes of motion pattern, global rotational stiffness curves, and animations of the disc and facet forces. This model was used to evaluate different mounting configurations for axial rotation testing and to identify a set of end constraint conditions that produced physiologic responses during axial rotational loading. This model was also used to simulate the biomechanical responses of single-level cervical fusion.
\end{abstract}

The single-level fusion was found to produce increased motion compensation at the adjacent segments during flexion and extension. Greater increases in the disc forces were found in the spinal level superior to the fusion during flexion and inferior to the fusion during flexion extension. This model was also used to study of the biomechanical effects of different design features for cervical disc arthroplasty. A constrained spherical joint placed at the disc level significantly increased facet loads during extension. Lowering the rotational axis of the spherical joint into the subjacent body also caused a marginal increase in facet loading during flexion, extension, and lateral bending. Un-constraining the spherical joint to a plane at the disc level minimized facet load build up.

The virtual model bridges the gap between the cadaveric-based in vitro tests and clinicalbased experimental studies to further the research and educational knowledge of cervical spine biomechanics.

\section{Document Type}

Thesis

Degree Name

Doctor of Philosophy (Medical Science)

Program

Biomedical Engineering

Research Advisor

Denis DiAngelo, Ph.D.

\section{Keywords}

cervical spine, dynamic multibody model, virtual simulation, axial rotation, in vitro testing; mounting configuration, adjacent segment disease, biomechanics, fusion, disc arthroplasty 


\section{Subject Categories}

Medical Biotechnology | Medical Sciences | Medicine and Health Sciences | Musculoskeletal, Neural, and Ocular Physiology | Other Medical Sciences 


\title{
A Virtual Model of the Human Cervical Spine for Physics-based Simulation and Applications
}

\author{
A Dissertation \\ Presented for \\ The Graduate Studies Council \\ The University of Tennessee \\ Health Science Center
}

\begin{abstract}
In Partial Fulfillment
Of the Requirements for the Degree

Doctor of Philosophy

In the Joint Graduate Program in Biomedical Engineering

From The University of Tennessee

and

The University of Memphis
\end{abstract}

By

Hyung Soo Ahn

May, 2005 


\section{DEDICATION}

This dissertation is dedicated to my parents,

Dong Il Ahn and Choon Ja Yang,

and to my wife, Mi Kyung Ye

for their love and support. 


\section{ACKNOWLEGEMENTS}

I would like to thank my advisor, Dr. Denis J. DiAngelo for giving me the opportunity to work on this project and for encouragement and guidance. I would also like to express my gratitude to other committee members, Dr. James T. Robertson, Dr. Michael Yen, Dr. Richard J. Kasser, and Dr. Teong E. Tan for their great advice based on years of experience. I am also grateful to the members of Joint-Implant Biomechanics laboratory: Henry Bonin, John Judd, Brian Kelly, Brian Morrow, John Schwab, Charles Sullivan, and Jun Yan.

I also would like to gratefully acknowledge the care and support of my father and mother, who always encourage me to pursue the Way. Above all, I would like to express my best appreciation to my wife, Mi Kyung Ye, for her love and understanding throughout the years. 


\begin{abstract}
Utilizing recent advances in computer technology, Our Biomechanics Laboratory have made an effort to integrate computer animation and engineering analysis software into biomedical research, specifically towards simulation and animation of in vitro experimentation of the human cervical spine in the virtual world. The objectives of this study were to develop a virtual model of the human cervical spine for physics-based simulation and to apply the virtual model to studies of different surgical procedures and instrumentation.
\end{abstract}

A process for creating an accurate virtual model of the human cervical spine was developed. The model consisted of seven vertebrae (C2-T1) connected with soft tissue components: intervertebral joint, facet joints, and ligaments. The soft tissue components were assigned nonlinear viscoelastic properties. The evaluation of the model included the percent contribution of rotation relative to global rotation, coupling behaviors, helical axes of motion pattern, global rotational stiffness curves, and animations of the disc and facet forces. This model was used to evaluate different mounting configurations for axial rotation testing and to identify a set of end constraint conditions that produced physiologic responses during axial rotational loading. This model was also used to simulate the biomechanical responses of single-level cervical fusion. The single-level fusion was found to produce increased motion compensation at the adjacent 
segments during flexion and extension. Greater increases in the disc forces were found in the spinal level superior to the fusion during flexion and inferior to the fusion during flexion extension. This model was also used to study of the biomechanical effects of different design features for cervical disc arthroplasty. A constrained spherical joint placed at the disc level significantly increased facet loads during extension. Lowering the rotational axis of the spherical joint into the subjacent body also caused a marginal increase in facet loading during flexion, extension, and lateral bending. Un-constraining the spherical joint to a plane at the disc level minimized facet load build up.

The virtual model bridges the gap between the cadaveric-based in vitro tests and clinicalbased experimental studies to further the research and educational knowledge of cervical spine biomechanics. 


\section{TABLE OF CONTENTS}

1 Introduction 1

1.1 Background on the computer modeling technique . $\quad$. $\quad$. $\quad$. $\quad$. 3

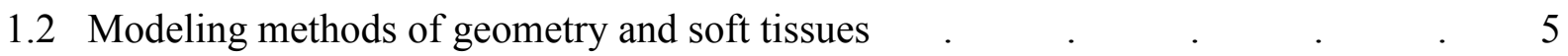

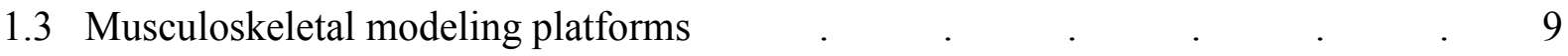

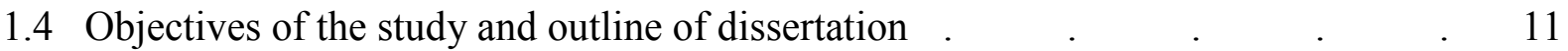

2 A Virtual Model of the Human Cervical Spine for Physics-based Simulation 13

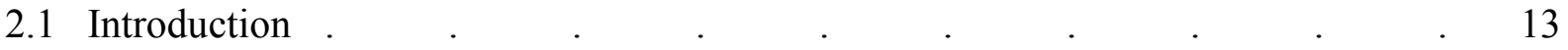

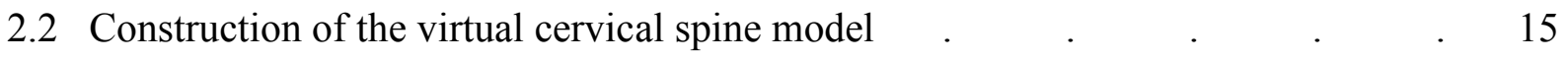

2.2.1 Anatomical description of the cervical spine . $\quad . \quad$. $\quad . \quad$. 15

2.2.2 The interconnecting joints and ligaments $\quad . \quad$. $\quad . \quad$. $\quad . \quad$. 17

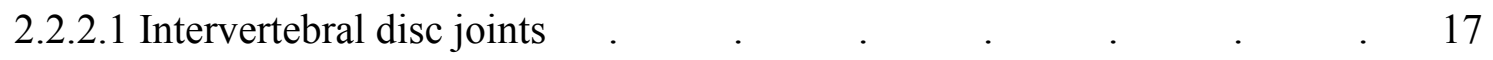

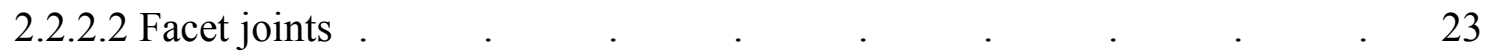

2.2.2.3 Ligaments . . . . . . . . . . . . . 24

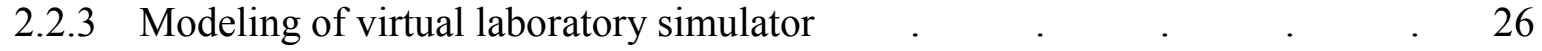

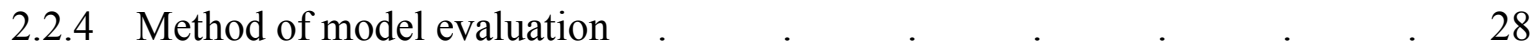

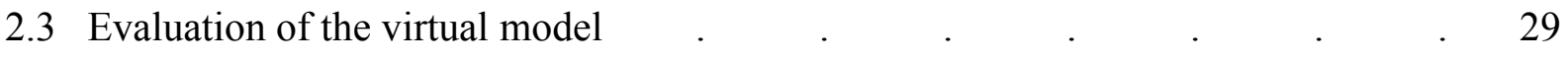

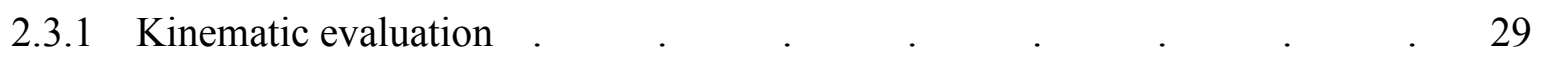

2.3.1.1 Relative motion segment unit rotations $\quad . \quad$. $\quad . \quad$. $\quad . \quad 29$

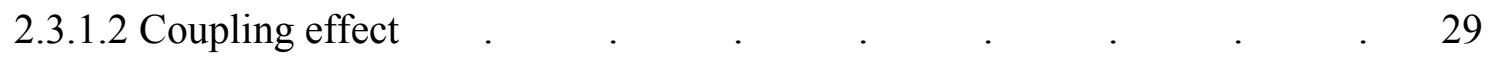

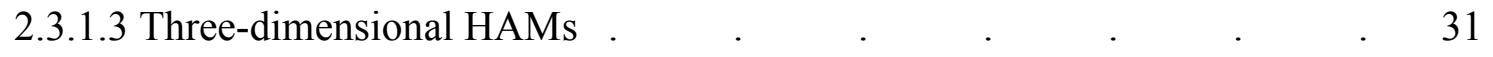

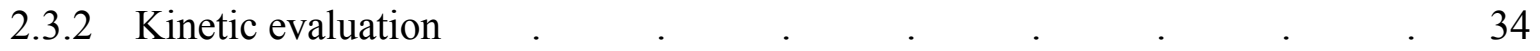

2.3.2.1 Global rotational stiffness .

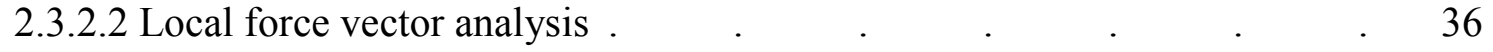

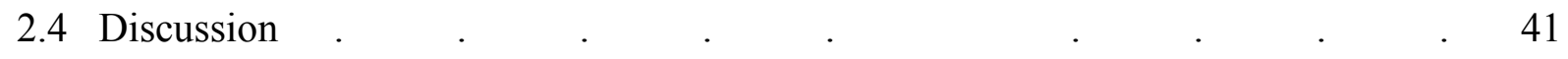

2.4.1 Flexion, extension, and lateral bending $\quad . \quad$. $\quad . \quad$. $\quad . \quad$. 41

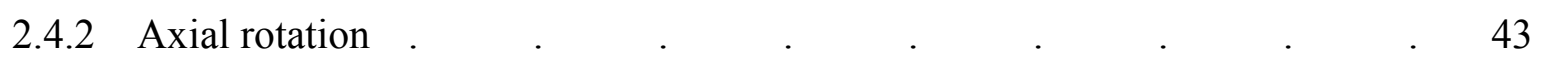

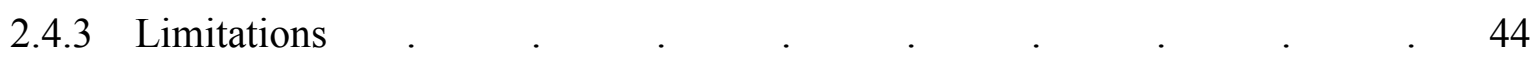

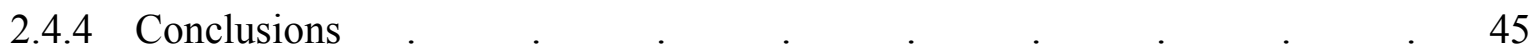

3 An Improved Axial Rotational Testing Protocol for the Cervical Spine 46

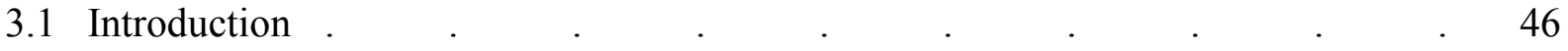




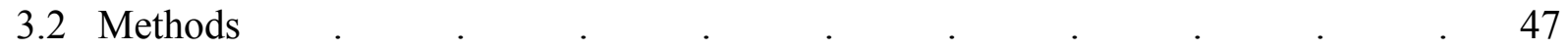

3.2.1 Development of the virtual computer simulation model $\quad$. $\quad$. $\quad$. 47

$\begin{array}{llllllllllll}\text { 3.2.2 } & \text { Modified in vitro axial testing protocol } & \text {. } & \text {. } & \text {. } & & 4\end{array}$

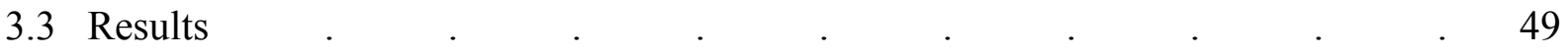

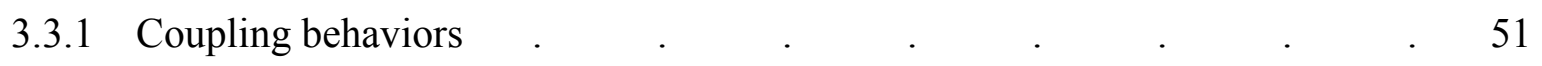

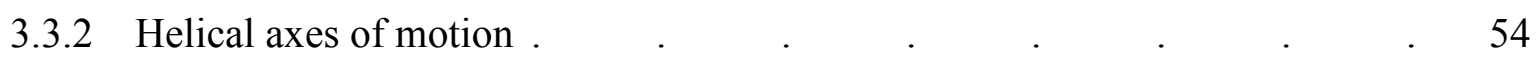

3.3.3 Global rotational stiffness curves $\quad . \quad$. $\quad . \quad$. $\quad . \quad$. $\quad . \quad 54$

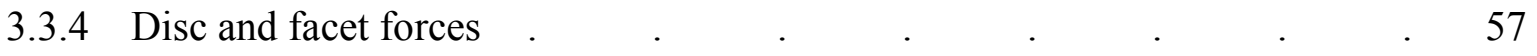

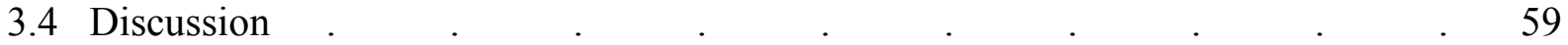

4 Biomechanics of Single-level Cervical Fusion Using a Virtual Dynamic Spine $\begin{array}{ll}\text { Model } & 63\end{array}$

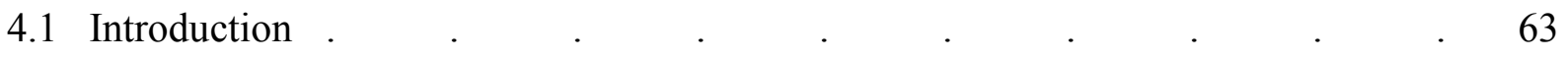

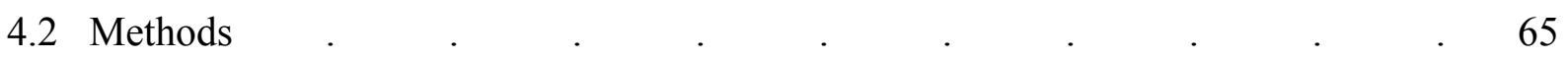

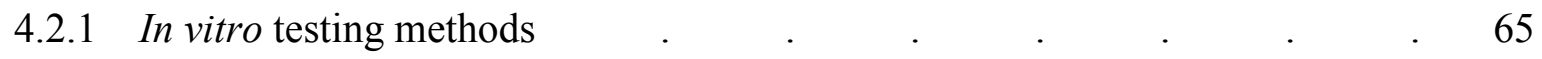

4.2.2 Construction of the model and simulation task. $\quad . \quad$. . . . 66

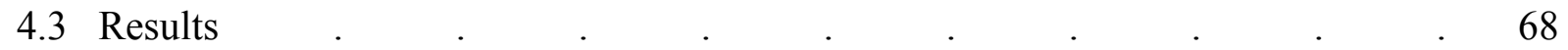

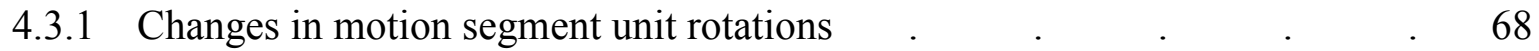

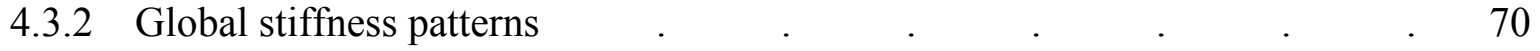

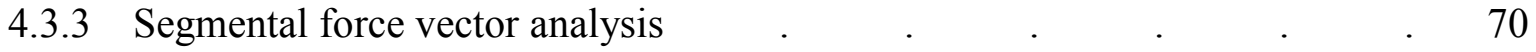

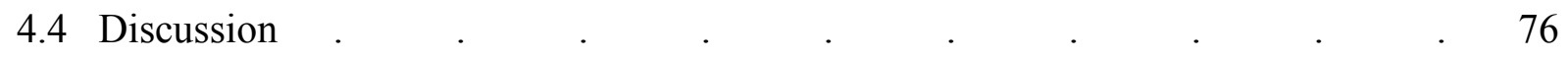

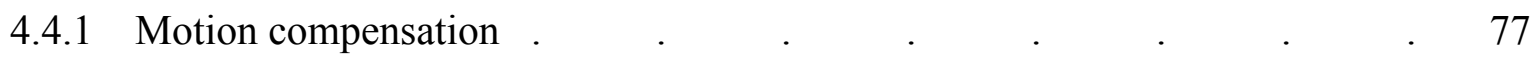

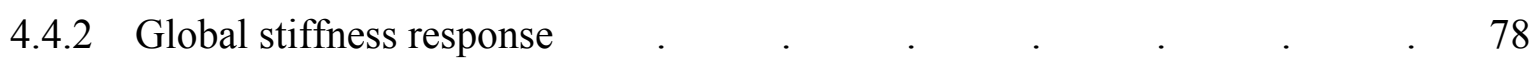

4.4.3 Segmental disc and facet joint force vectors . . . . . . . . . $\quad$. 78

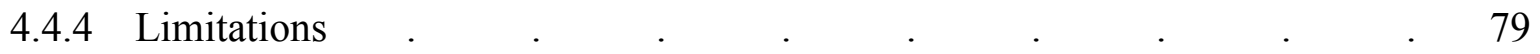

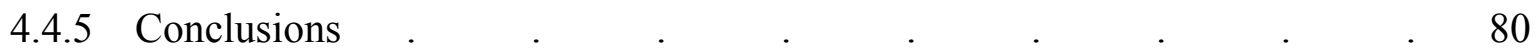

\section{Dynamic Simulation of the Effect of Different Disc Arthroplasty Designs on} Cervical Spine Biomechanics

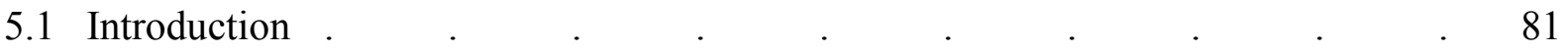

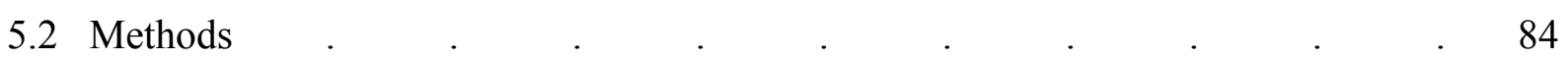

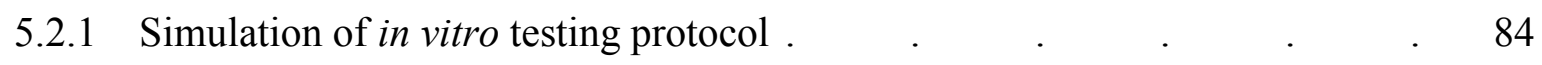

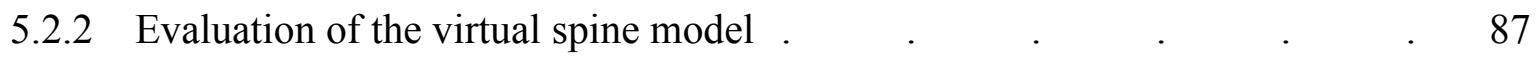

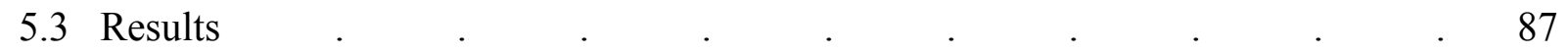

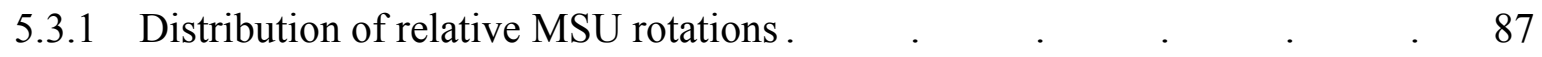

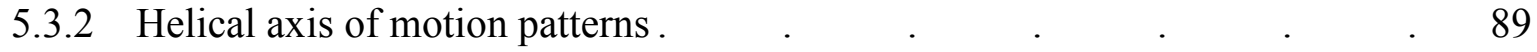




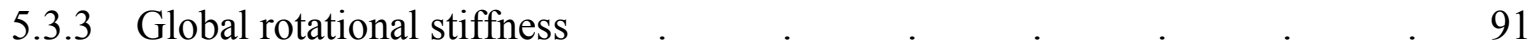

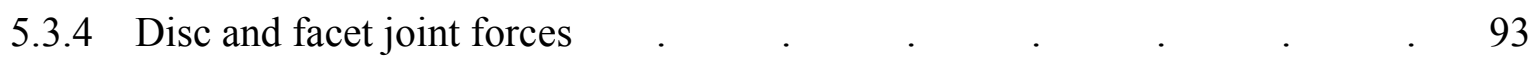

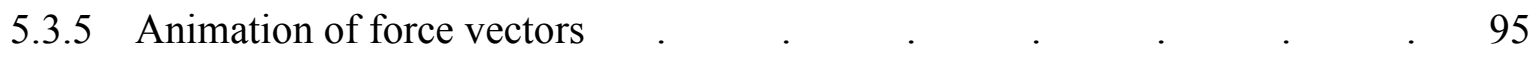

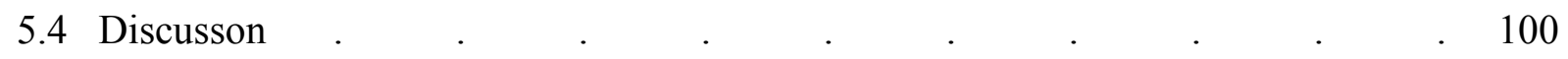

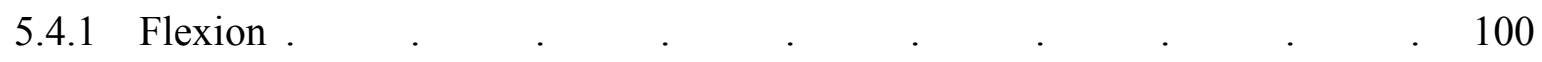

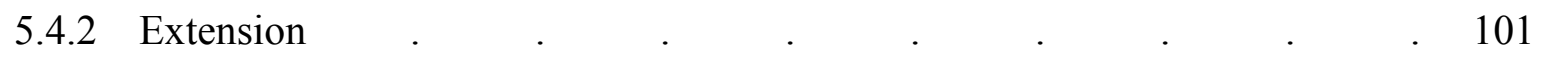

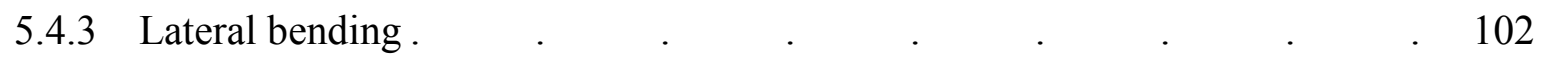

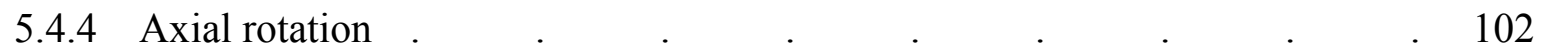

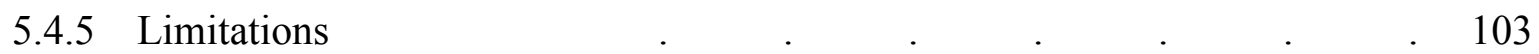

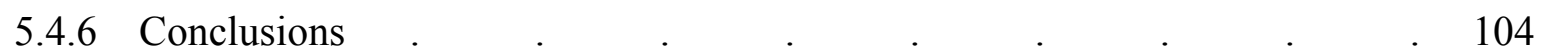

6 Discussion $\quad 105$

6.1 Development and evaluation processes of the virtual model. $\quad$. . . 105

6.2 A simulation study of axial rotational testing protocol $\quad . \quad$. $\quad . \quad$. 107

6.3 A simulation study of single-level spine fusion $\quad . \quad$. $\quad . \quad$. $\quad . \quad 108$

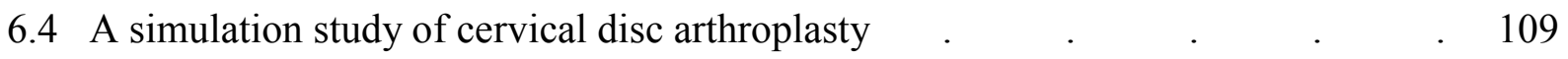

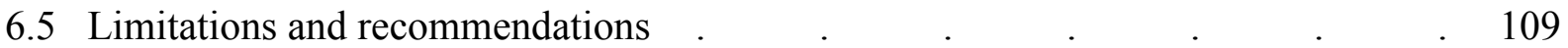

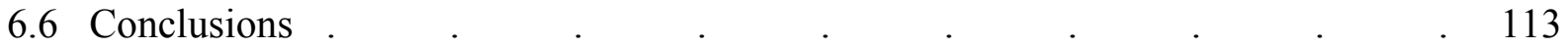

7 Suggested Future Work $\quad 114$

$\begin{array}{ll}\text { List of References } & 116\end{array}$

Vita

126 


\section{LIST OF TABLES}

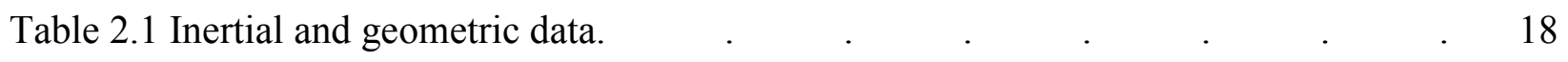

Table 2.2 Coefficients for global rotational stiffness properties (C2-T1). . 22

Table 2.3 Statistical evaluations of global stiffness curves. . $\quad . \quad$. $\quad . \quad$. 37

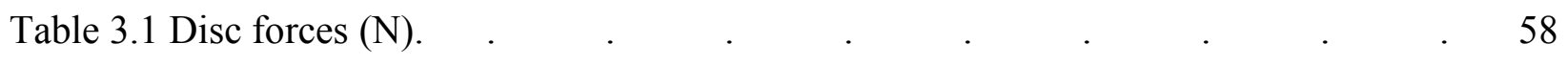

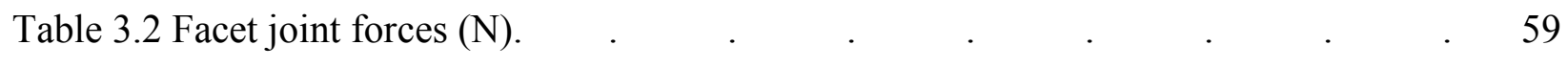

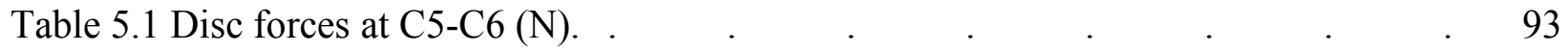




\section{LIST OF FIGURES}

Figure 2.1 The creation process of the human cervical vertebrae model. $\quad$. 16

Figure 2.2 The three-dimensional coordinate system for intervertebral disc joints

and facet joints, used to define loading orientation and relative motions. $\quad 18$

Figure 2.3 The virtual simulation model of sub-axial cervical spine. . . $\quad$. 19

Figure 2.4 Averaged moment-rotation data (C2-T1) from six specimens. . . $\quad 22$

Figure 2.5 The stiffness (k) vs. strain (e) response of a ligament. $\quad . \quad$. $\quad 25$

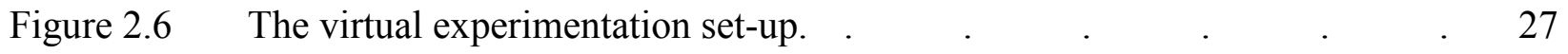

Figure 2.7 The comparisons of the distribution of relative motion changes: between

our testing protocol, in vivo, and simulation. . $\quad . \quad$. . . . $\quad .30$

Figure 2.8 Coupled behaviors between lateral bending and axial rotation. $\quad$. $\quad$. 32

Figure 2.9 Animation frames of HAMs during flexion, extension, lateral bending, and

axial rotation loading. . $\quad . \quad$. $\quad . \quad$. $\quad . \quad . \quad$. $\quad . \quad . \quad 33$

Figure 2.10 Axis-disc angle during lateral bending and axial rotation loading. . . $\quad 35$

Figure 2.11 Kinetic evaluation of global rotational stiffness properties. . _ . $\quad$. 37

Figure 2.12 Animation frames of local forces and the plots of compressive forces versus

global rotation during (A) flexion and (B) extension. . . . . 38 
Figure 2.13 Animation frames of local forces and the plots of compressive forces versus

global rotation during (A) lateral bending and (B) axial rotation. . . . $\quad 40$

Figure 3.1 Schematic of the virtual experimental set-up for axial rotation tests.. $\quad$. 48

Figure 3.2. Modified testing set-up for in vitro axial rotation tests. _ . . . . 50

Figure 3.3 The coupling pattern between different end constraint conditions during axial

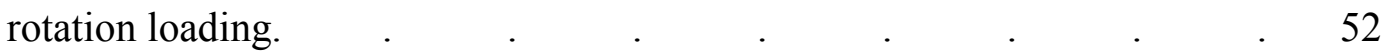

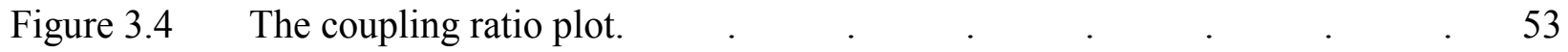

Figure 3.5 Animation frames of HAM vector analysis. . _ . . . . . $\quad$. 55

Figure 3.6 Global rotational stiffness curves for the simulation and in vitro tests. . 56

Figure 3.7 Animation frames of the disc and facet joints force vectors. . . . . 58

Figure 4.1 The in vitro experimental testing apparatus and animation frames of the virtual

experimentation of a single-level fusion at the C5-C6 level. . $\quad . \quad$. 67

Figure 4.2 The change in percent contribution of rotation before and after C5-C6 fusion

between the in vitro testing and simulation studies. . $\quad . \quad$. $\quad$. 69

Figure 4.3 Typical in vitro global rotational stiffness curves before and after fusion

(C5-C6).

Figure 4.4 The simulation results of global rotational stiffness curves before and after

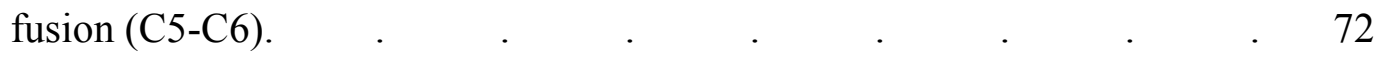


Figure 4.5 The differences in magnitude of the disc translational force before and after

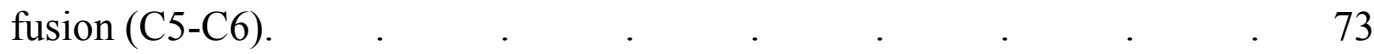

Figure 4.6 Animation frames of segmental disc and facet translational forces. . . $\quad 75$

Figure 5.1 Three different design variations of the prosthetic disc. $\quad . \quad$. $\quad$. 86

Figure 5.2 Relative MSU contributions for the intact spine and prosthetic disc designs

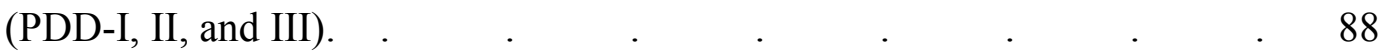

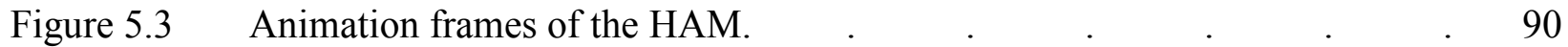

Figure 5.4 The applied moment versus global rotation curves. . _ . . . . $\quad$. 92

Figure 5.5 The facet forces (FF) at the C5-C6 MSU. $\quad . \quad$. $\quad . \quad$. $\quad . \quad$. $\quad 94$

Figure 5.6 Animation frames of the disc and facet joint force vectors at C5-C6 MSU for intact and implanted spines during flexion. $\quad . \quad$. $\quad$. $\quad$. $\quad$. $\quad$. $\quad 96$

Figure 5.7 Animation frames of the disc and facet joint force vectors at C5-C6 MSU for intact and implanted spines during extension. $\quad . \quad$. $\quad . \quad$. $\quad$. 97

Figure 5.8 Animation frames of the disc and facet joint force vectors at C5-C6 MSU for intact and implanted spines during lateral bending. . $\quad$. $\quad$. $\quad$. 98

Figure 5.9 Animation frames of the disc and facet joint force vectors at C5-C6 MSU for intact and implanted spines during axial rotation. $\quad . \quad$. $\quad . \quad$. $\quad 99$ 


\section{Chapter 1}

\section{Introduction}

Biomechanics of the human cervical spine, with its complex kinematics and geometry, remains one of the more challenging areas of studies of the human musculoskeletal system. Many researchers have developed simulation models of the cervical spine to better understand these complexities. Panjabi (1998) classified four techniques for modeling cervical spine biomechanics: physical models, in vitro models, in vivo models, and computer models. Physical models, made of non-biological material, are often used for testing spinal instrumentation when only the device is evaluated. In vitro models, which consist of animal or human cadaver specimens, are useful in providing a basic understanding of the cervical spine. In vivo models, which consist of mostly animals or sometimes human volunteers, provide a means for modeling the living phenomena. Computer (numerical) models are a set of mathematical equations that incorporate both the geometry and physical properties of the representing structure (Panjabi, 1998).

Among the four types of modeling techniques, computer models have several features that make them more appealing than other types of models. First, an infinite number of computer models can be developed and tested for different conditions (Fagan et al., 2002). Second, unlike 
biological tissue, the primary characteristic of a computer model is maintained after exhaustive testing and the same model can be used repeatedly for multiple experiments (Fagan et al., 2002). Third, computer models provide information that cannot be easily obtained using other types of models, such as load distributions within soft tissues, internal stresses, joint reaction forces, and muscle force analyses (Fagan et al., 2002). Fourth, once an accurate computer model has been developed and validated appropriately, simulations can be performed quickly and at low cost in diverse situations, such as injury, surgery, and dynamic motion (Chao, 2003; Nigg et al., 1999). Fifth, graphical animation of the experimentation helps enhance the understanding of the underlying mechanisms (Chao, 2003; Cripton et al., 2001).

The next section provides background information of major types and progressions for computationally modeling the cervical spine. Section 1.2 presents published modeling techniques used for geometric construction of cervical vertebral bodies and the interconnecting soft tissues. A review of currently available musculoskeletal modeling platforms is described in Section 1.3. Section 1.4 introduces the objectives of this research work and an outline of this dissertation. 


\subsection{Background on the computer modeling technique}

Several computer models have been created to study biomechanics of the human cervical

spine. In the literature mainly two types of computer modeling techniques have been used for the biomechanical study of the cervical spine: finite element models and multi-body dynamic models. Finite element models, which are constructed using many small geometric elements, have been used in structural analyses to study the internal stresses of an object under external loads. Finite element models are able to represent complex geometries and simulate material behavior in detail. Several three-dimensional cervical spine models have been published with increasing accuracy and complexity (Goel and Clausen, 1998a; Kumaresan et al., 1997a; 1997b;

1998; 1999b; Maurel et al., 1997; Ng et al., 2003; Ng and Teo, 2001; Teo and Ng, 2001a; 2001b;

Voo et al., 1997; Yoganandan et al., 1996). Although the finite element models show reasonable response under quasi-static loads, the simulations of kinematics and dynamic responses remain inaccurate (Bogduk and Yoganandan, 2001).

Multi-body dynamic models are mathematical models composed of rigid bodies (the cervical vertebrae) and spring-damper elements (interconnecting soft tissue). Mathematical equations representing the behavior of spring-damper elements govern the kinematic and kinetic response of the models to externally applied forces. These multi-body models are suitable for kinematic 
and dynamic analyses. Deng and Goldsmith (1987), De Jager (1996), and van der Horst (2002) developed multi-body cervical spine models to study the human head-neck response to impact loading (i.e., whiplash injury). Although these multi-body models used rigid bodies to represent the cervical vertebrae, the model response was accurate enough to study the kinematics and kinetic responses to impact loading. Simulation of vertebrae deformation simulation is needed for studying fracture mechanics or severe loading conditions (van der Horst, 2002). Recently, to achieve a more realistic kinematics and dynamic simulation with computer models, investigators have developed multi-body dynamic simulation engines that are based on the laws of physics. The advanced models generate animations of physical events of known mechanical systems (Coutinho, 2001; Porcher Nedel and Thalmann, 2000). Physics-based modeling is particularly attractive because one can run interactive simulations in real-time (García de Jalón and Bayo, 1994). Furthermore, the simulations can be extended to multiple-domain analyses (Coutinho, 2001). For example, dynamic motion simulation can be combined with stress analysis.

Along with the develpoment of these modeling and simulation techniques, there has also been significant advancement in biomedical image acquisition and processing technology. Threedimensional visualization of medical images has provided valuable information to clinicians and it is used to construct three-dimensional human anatomy for educational purposes. These biomedical images contain topological information that can not only be used in this descriptive 
way, but also to generate models for structural manipulation/analysis and dynamic simulation

(Beylot et al., 1996).

With computer modeling/simulation techniques and advancements in medical imaging technology, an effort has been made to integrate computer graphics, medical imaging technology, computer aided design, and engineering analysis programs into biomedical research (Chao, 2003; Delp and Loan, 1995). The integration of computer graphics and analysis tools provides a wide spectrum of biomechanical analyses in a virtual environment (Chao, 2003).

However, accurate modeling and simulation of the musculoskeletal system is still a challenging task, particularly for the complex structures of the human cervical spine (Delp and Loan, 1995).

\subsection{Modeling methods of geometry and soft tissues}

The two main steps of computer modeling are the generation of vertebral geometry and the definition of interconnecting joints and soft tissue components. Two distinct approaches for the generation of geometry have been identified in literature.

One approach is parameteric modeling that addresses the variability of biologic specimens and individualizes the model to fit different morphologies (Maurel et al., 1997). To obtain spatial parameter values, one uses a coordinate-measuring techniques, computerized tomography 
(CT) measurements, and calculations based on physiological assumptions (Maurel et al., 1997).

Although there seems to be no consensus in defining parameter criteria essential for geometry reconstruction, the parametric modeling approach has some merit, such as its ability to study the relationship between geometric parameter changes and mechanical responses of the cervical spine (Maurel et al., 1997).

Another prominent approach for geometric reconstruction of the vertebrae is to model the cervical vertebrae as realistically as possible using various sources of data, such as computerized tomography images (Goel and Clausen, 1998a; Kumaresan et al., 1999b; Voo et al., 1997; Yoganandan et al., 1996) and three-dimensional digitizers (Ng and Teo, 2001; Teo and Ng, 2001b). Since the geometry significantly affects the mechanical and dynamic behavior of the cervical spine (Yoganandan et al., 1991), accurate representation of geometry is an important step for more accurate computer modeling and simulation. In some advanced models, cryomicrotome sections were used to achieve accurate boundaries for soft tissue structures incorporated in the process of cervical vertebrae geometry reconstruction (Kumaresan et al., 1999b; Voo et al., 1997).

The next and more important step was to define the interconnecting joints and soft tissue components. A variety of structures connecting vertebrae can be classified as soft tissue structures, which include facet joints, intervertebral discs, ligaments, and muscles (Yoganandan 
et al., 2001). The role of these soft tissue structures is to provide flexibility to the vertebral column while maintaining the integrity of the column (White and Panjabi, 1990; Yoganandan et al., 2001). The mechanical and dynamic responses of the cervical spine model are more sensitive to the properties of soft tissue structures than hard tissue structures (Kumaresan et al., 1999a).

Geometric configurations and material properties of the facet joints strongly influence the movement of the cervical spine in the response to the external forces (Onan et al., 1998a; 1998b; White and Panjabi, 1990). To represent the facet joint and its function in computer models, both gap (Goel and Clausen, 1998a; Maurel et al., 1997) and solid (Kumaresan et al., 1997b; Yoganandan et al., 1996) elements have been used. The motion of the joint contact interfaces has been approximated by spring-damper (van der Horst, 2002) or sliding contact element (De Jager, 1996; Teo and Ng, 2001a). An advanced approach to model the detailed facet joint incorporated incompressible fluid elements to represent the synovial fluid (Kumaresan et al., 1998).

To model the anatomic components of the intervertebral discs, two or three elements have been used. Solid elements (Kumaresan et al., 1999b; Teo and Ng, 2001a) and incompressible fluid (Goel and Clausen, 1998a; Kumaresan et al., 1999b) elements have been used to simulate the annulus ground substance and the nucleus pulposus, respectively. Crossed cable (Maurel et 
al., 1997), fiber reinforcement (Goel and Clausen, 1998a), and rebar (Kumaresan et al., 1999b)

elements have been used to simulate annulus fibers. In multi-body modeling approaches, the linear behavior of the intervertebral disc (six degree-of-freedom) was modeled by parallel connections of springs and dampers (De Jager, 1996; van der Horst, 2002).

The ligaments of the cervical spine have been modeled as straight line (De Jager, 1996; van der Horst, 2002) and cable (Goel and Clausen, 1998a; Kumaresan et al., 1999b; Maurel et al., 1997; Teo and Ng, 2001a) elements. The mechanical properties of the ligaments have been modeled both linearly (Maurel et al., 1997) and non-linearly (De Jager, 1996; Goel and Clausen, 1998a; Kumaresan et al., 1999b; van der Horst, 2002).

Active and passive muscle forces have an important role in the movement and stability of the cervical spine. However most current computer models of the cervical spine, especially finite element models, lack these muscle elements. In some multi-body modeling approaches, passive muscle behavior has been modeled by non-linear force-strain (Deng and Goldsmith, 1987; De Jager, 1996) or through a stress-strain relation (van der Horst, 2002). Active muscle behavior has been simulated using a function imbedded in the specific modeling software (De Jager, 1996; van der Horst, 2002). 


\subsection{Musculoskeletal modeling platforms}

Commercial multi-body simulation programs, such as ADAMS (MSC software, Santa Ana, CA) and DADS (LMS International, Leuven, Belgium), can formulate dynamic equations of motion and solve them numerically to study the human musculoskeletal system. However, without the aid of computer graphics software permitting three-dimensional visualization, it is difficult to interpret the result of simulation of musculoskeletal models. Currently a few distinctive software platforms have been developed to provide interactive graphic-based musculoskeletal model/simulation. Virtual Interactive Musculoskeletal System (VIMS), Software for Interactive Musculoskeletal Modeling (SIMM), LifeMod, and AnyBody Modeling System are four notable software based musculoskeletal model/simulations currently used for research.

VIMS, developed at Orthopaedic Biomechanics Laboratory at Johns Hopkins University, is a versatile musculoskeletal simulation tool providing libraries of models. The libraries include full skeletal models (no relative motion within spine, trunk, hand, wrist, foot), shoulder, pelvis/hip musculoskeletal models, as well as hip joint replacement and external fixator models (Chao, 2003). Since the musculoskeletal models of VIMS do not have a muscle-tendon unit with force- 
generating properties, the models made by VIMS are mainly for kinematics and motion

animation.

SIMM, reported by Delp and Loan (1995), is a graphics-based software developed to primarily study joint kinematics and muscle force/moment properties. For dynamic simulation, SIMM needed to be used in conjunction with SD/Fast, a dynamics engine (Delp, 2000).

Although SIMM allows users to characterize the kinematics of any joint, the kinetic joint models are not directly available in SIMM models (Delp, 2000). Vasavada et al. (1998) developed a neck model using SIMM software to study the influence of muscle morphometry and moment arms on the moment-generating capacity of human neck muscles.

Other available human modeling programs are LifeMod (The Biomechanics Research Group, San Clemente, CA) and the AnyBody Modeling System (AnyBody Technology, Aalborg, Denmark). These modeling programs are specialized for human musculoskeletal modeling and provide ready-made geometry and joint models.

In this project, a more general engineering program, VisualNastran 4D (MSC software, Santa Ana, CA), was used to develop a customized spine model to simulate complex non-linear viscoelastic behaviors. 


\subsection{Objectives of the study and outline of dissertation}

The rationale for this project was that a computer model of the human cervical spine can contribute to understanding the complex cervical spine biomechanics in special situations such as arthrodesis of cervical spine motion segment unit or artificial cervical spine arthroplasty. To fully understand and improve the clinical results, it was necessary to understand how these procedures affect the loads transferred along the adjacent segments in a fused condition or the effect on other spinal structures of a mechanical joint replacing a disordered disc. However, the interpretation of the simulation results of the computer model must be performed carefully and evaluated by experimental data. To evaluate the model appropriately, it was ideal to simulate the computer model in the same experimental condition. Fortunately, it was possible to have enough information to model the in vitro experimentation protocol of our Biomechanics Laboratory and to obtain raw data for the comparison.

The majority of audiences in human cervical spine biomechanical research are clinicians who are not familiar with engineering concepts and terminology. Still researchers have to be inspired from these clinicians' work and inform them new methods to improve the outcome of their treatment. The graphical presentation of the experimental results can be an optimal way for this interdisciplinary communication. 
The objectives of this study were 1) to develop a graphics-oriented multi-body model of the human cervical spine, 2) to build a virtual laboratory simulator for kinematic and kinetic testing using physics-based dynamic simulation techniques, and 3) to apply the model to experimentally and clinically important situations such as improvement of a in vitro testing protocol and the biomechanical effects of cervical spine fusion or arthroplasty.

Chapter 2 gives a detailed description of the technique for the reconstruction of geometry of the human cervical spine, the definition of the interconnecting soft tissue structures, and the model evaluation. Chapter 3 presents the simulation study of different mounting configurations for axial rotation testing to identify a set of end conditions that produce physiologic responses during axial rotational loading. Chapter 4 presents the simulation study of single-level (C5-C6) fusion to investigate the mechanical effects on adjacent segments. Chapter 5 presents the simulation study of the artificial cervical discs at the level of C5-C6 to determine the kinematic and kinetic effect on the surrounding structures. Chapter 6 gives conclusive discussion by summarizing the main findings of this study. The limitations and recommendations for enhancing the model are also presented. Suggestions for future work are given in Chapter 7. 


\section{Chapter 2}

\section{A Virtual Model of the Human Cervical Spine for Physics-based Simulation}

\subsection{Introduction}

With emerging computer technology, there have been efforts to integrate computer aided design (CAD), computer animation techniques, and medical imaging into the field of biomedical research (Chao, 2003; Delp and Loan, 1995). Medical images can be reconstructed to generate three dimensional computer models and used for structural manipulation and dynamic simulation in CAD and engineering analysis programs (Beylot et al., 1996). Since computerized models have appealing aspects over conventional laboratory experimentation (Fagan et al., 2002), several computer models have been developed to study the biomechanics of the human cervical spine (De Jager, 1996; Goel and Clausen, 1998; Kumaresan et al., 1997a; 1998; 1999b; Maurel et al., 1997; Ng et al., 2003; Ng and Teo, 2001; Teo and Ng, 2001a; 2001b; van der Horst, 2002; Voo et al., 1997; Yoganandan et al., 1996). Most computer models of the human cervical spine were developed using the finite element modeling (FEM) technique. Recently, physics-based dynamic simulations of multi-body systems have gained increasing acceptance within the fields 
of computer graphics and mechanical engineering as a way of achieving realistic animation and accurate simulations of complex mechanical systems (Coutinho, 2001). Physics-based computer simulation applies the laws of physics to solid bodies with defined material properties to achieve a more realistic kinetic and kinematic response (Coutinho, 2001; Porcher Nedel and Thalmann, 2000). As opposed to other computer modeling techniques (e.g., FEM), physics-based dynamic simulation is particularly attractive because of the ability for interactive simulation with the physical world even in real-time (García de Jalón and Bayo, 1994). These techniques can be combined to create a virtual simulator for kinematics and kinetics testing of the human cervical spine. An accurate virtual model would complement tissue-based in vitro studies of cervical spine biomechanics by providing a consistent test-bed without variability and by a reduction in the cost of cadaver tissue testing (Fagan et al., 2002; Nigg et al., 1999). In addition, the graphical animation of virtual experiments allows intuitive interpretation of the data, independent of the viewer's expertise (Chao, 2003; Cripton et al., 2001).

The objectives of this study were to develop a graphics-oriented multi-body model of the human cervical spine and to build a virtual laboratory simulator for kinematic and kinetic testing of the model using physics-based dynamic simulation techniques. 


\subsection{Construction of the virtual cervical spine model}

\subsubsection{Anatomical description of the cervical spine}

The geometry of the skull, cervical vertebrae, and upper torso (clavicles, scapulae, ribs and sternum) was created from axial computer tomography (CT) images available from the National Library of Medicine's Visible Human data set (Ackerman and Banvard, 2000).

Using a manual segmentation/tagging procedure, contour points outlining the boundaries of each vertebra were derived in each two-dimensional slice by using a medical image processing software (SliceOmatic 4.2, Tomovision, Montreal, Canada) (Figure 2.1). The tagged bony contours of each cervical vertebra were converted into DXF (Drawing eXchange Format) file format and imported into volumetric surface modeling software (Geomagic Studio 5, Raindrop Geomagic, Research Triangle Park, NC). Digital smoothing algorithms in combination with manual editing techniques within this software allowed excellent surface reproduction from the point cloud data (Figure 2.1). The surface models were converted into STL (STereo Lithography) file formats and imported into VisualNastran 4D (MSC software, Santa Ana, CA), a physics-based dynamic simulation software. Since the volume rendered images retained their positional information with respect to the other vertebral bodies (Figure 2.1), the reconstructed cervical spine column maintained its original geometric relationship. 
(A)
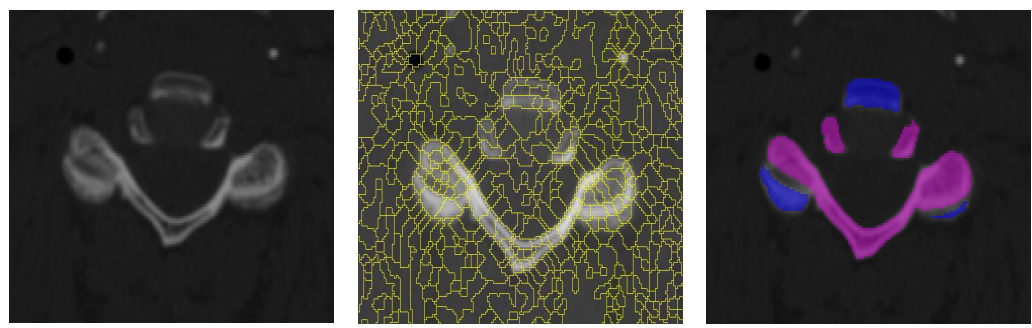

(B)

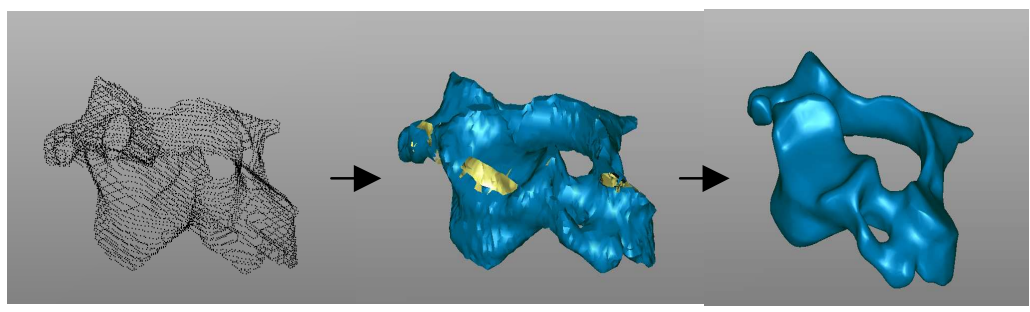

(C)
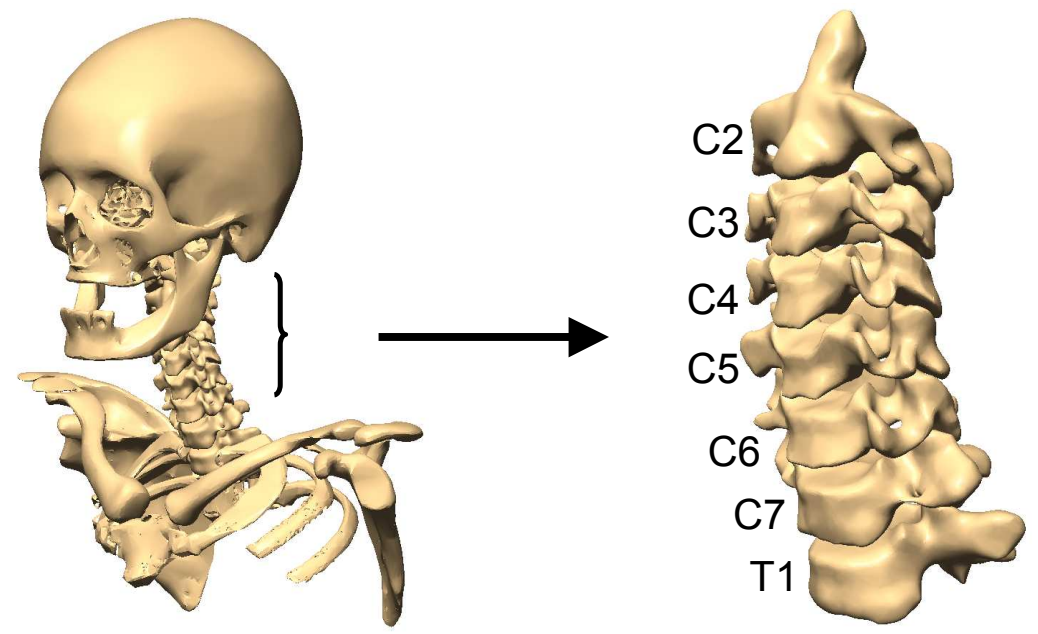

Figure 2.1 The creation process of the human cervical vertebrae model. (A) With the manual segmentation/tagging procedure, contour points outlining the boundaries of each vertebra were derived in each slice using SliceOmatic. (B) The tagged bony contours of each cervical vertebra were imported into Geomagic Studio. Digital smoothing algorithms in combination with manual editing techniques within this software allowed excellent surface reproduction from the point cloud data. (C) The surface models were imported into VisualNastran 4D. Since all the volume rendered images retained the original positional information, the final reconstructed model retained the original positional relationship of the cervical spine. 
In this study, seven rigid bodies from C2 vertebra to $\mathrm{T} 1$ inclusive, were used to simulate a harvested cadaveric sub-axial cervical specimen. The mass, the moment of inertia, and the position of the center of mass for the vertebral bodies were calculated by VisualNastran 4D software and are listed in Table 2.1. Three-dimensional coordinate systems were assigned and located at each intervertebral disc joint and facet joint and used to define the localized loads and displacements between each vertebrae (Figure 2.2).

\subsubsection{The interconnecting joints and ligaments}

The joints linking adjacent vertebrae were modeled as a 'triple joint complex' comprised of: (A) intervertebral disc joints in anterior region, (B) two facet joints in posterior region, and (C) surrounding ligament structure (Figure 2.3).

\subsubsection{Intervertebral disc joints}

The intervertebral disc joints were modeled as load-based joints with six degrees of freedom having non-linear viscoelastic properties defined by stiffness data from in vitro experiments in our Biomechanics Laboratory and data from the literature. Since the crescentic anterior portion of the annulus is much thicker than the posterior portion, the nucleus is located a somewhat posteriorly rather than centrally within the disc (Mercer and Bogduk, 1999). Therefore, the 
Table 2.1 Inertial and geometric data.

\begin{tabular}{|c|c|c|c|c|c|c|}
\hline \multirow{2}{*}{$\begin{array}{c}\text { Vertebral } \\
\text { body }\end{array}$} & \multirow{2}{*}{$\begin{array}{l}\text { Mass } \\
(k g)\end{array}$} & \multicolumn{3}{|c|}{ Moment of inertia $\left(\mathrm{kgmm}^{2}\right)$} & \multirow{2}{*}{$\begin{array}{l}\text { Center of mass position } \\
\text { relative to T1 center of mass } \\
(\mathrm{mm}) \text { (X: right, Y: anterior, } \\
\text { Z: upward) }\end{array}$} & \multirow{2}{*}{$\begin{array}{l}\text { Facet } \\
\text { joint } \\
\text { angle } \\
\text { (deg)\# }\end{array}$} \\
\hline & & $I_{x x}$ & $I_{y y}$ & $I_{z z}$ & & \\
\hline $\mathrm{C} 2$ & 0.25 & 46.3 & 47.7 & 64.9 & $(3.29,32.61,93.80)$ & $\begin{array}{c}---- \\
-1\end{array}$ \\
\hline $\mathrm{C} 3$ & 0.26 & 23.7 & 48.9 & 65.3 & $(4.35,32.50,75.29)$ & 60 \\
\hline $\mathrm{C} 4$ & 0.27 & 23.8 & 57.7 & 72.2 & $(4.17,28.96,61.18)$ & 50 \\
\hline $\mathrm{C} 5$ & 0.26 & 24.3 & 61.2 & 73.4 & $(3.19,25.20,46.64)$ & 54 \\
\hline C6 & 0.29 & 38.8 & 62.7 & 79.0 & $(1.51,18.40,31.97)$ & 58 \\
\hline $\mathrm{C} 7$ & 0.41 & 72.1 & 89.2 & 132.8 & $(1.34,8.51,16.40)$ & 62 \\
\hline T1 & 0.49 & 93.5 & 127.5 & 174.4 & $(0.00,0.00,0.00)$ & 50 \\
\hline
\end{tabular}

\# The orientation angles of upper facet joint are relative to a horizontal plane of the intervertebral joint coordinate system. The angle of inclination of the facet joints in the sagittal plane increased cephalocaudally, except at the transition MSU levels (i.e., C2-C3 and C7-T1).

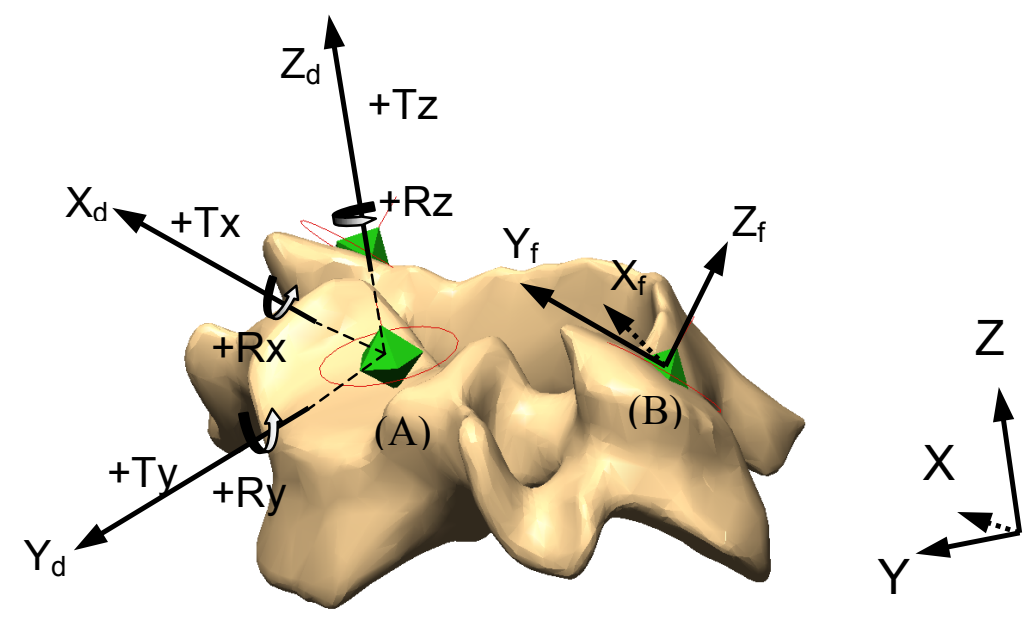

Figure 2.2 The three-dimensional coordinate system for intervertebral disc joints and facet joints, used to define loading orientation and relative motions. (A) The origin of the intervertebral joint and (B) The coordinate system for the facet joint. 


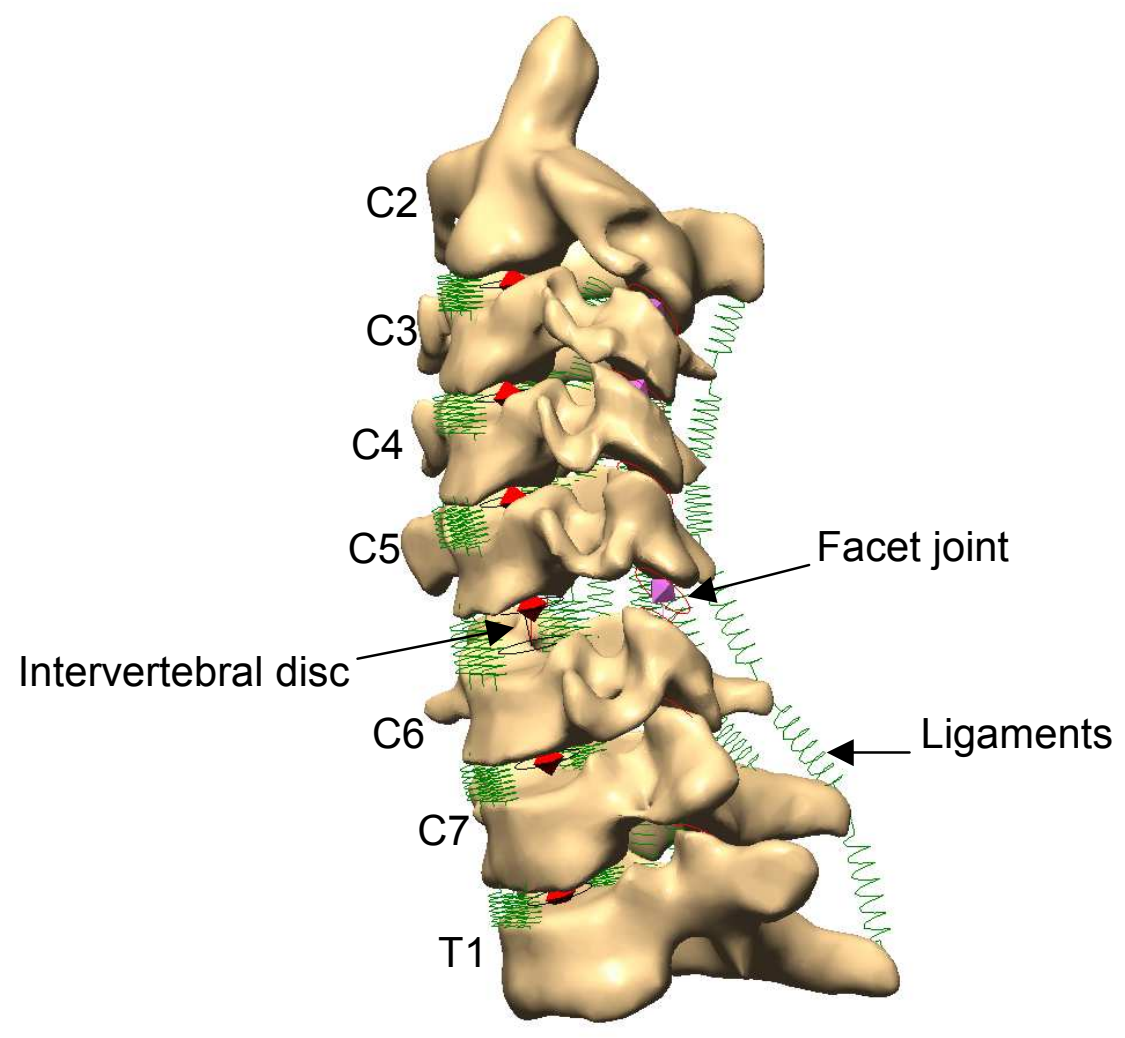

Figure 2.3 The virtual simulation model of subaxial cervical spine. The C5-C6 segment was detailed to show the constraints of intervertebral disc and facet joint. 
origin of the coordinate systems applied to each disc was set at mid-height of the posterior third of the intervertebral disc. The $X_{d}, Y_{d}$, and $Z_{d}$-axes were defined pointing to the right-laterally, anteriorly and cranially, respectively. Movement of each vertebra was defined by the coordinate system of the subjacent vertebral body (Figure 2.2).

To model viscous behavior, constant damping coefficients were applied. The values of the damping coefficient were 1Ns/mm for translations, 26Nmms/deg for rotations (De Jager, 1996; van der Horst, 2002).

To model elastic behavior, non-linear load displacement curves were assigned for each degree-of-freedom and each level of the intervertebral disc joints. Force-translation curves for the $\mathrm{X}_{\mathrm{d}}$ and $\mathrm{Y}_{\mathrm{d}}$ - directions (shear) were adapted and modified from the description of De Jager (1996) who used data from Panjabi et al. (1986) and Shea et al. (1991). Force-translation curves for the $Z_{\mathrm{d}}$-direction (tension-compression) were adapted directly from Shea et al. (1991).

To obtain a first approximation of the rotational stiffness parameters for each vertebral level, a global rotational stiffness response was first derived for each rotation mode (i.e., flexion, extension, lateral bending and axial rotation) using data from our Biomechanics Laboratory (Schwab, 2003). Since intervertebral disc moment-rotation curves were well represented by an initially linear region followed by a hyperbolic domain of increasing upward concavity (Merrill et al., 1984), a regression equation (1) was fitted to the global stiffness curves from Schwab 


$$
M=a+b \theta^{c}
$$

(2003), $M$ was the applied moment, $a, b$, and $c$ were constant coefficients, and $\theta$ was the measured global rotational displacement. Rotational stiffness data from six test specimens were normalized with respect range of motion, pooled and plotted for each mode of rotation. The pooled moment-rotation data points were fitted with equation (1) for each rotation mode using a curve fitting software (TableCurve v4, Systat Software, Richmond, CA) (Figure 2.4). The fitted constant coefficients for each mode are listed in Table 2.2. The regression equation provided for a good fit of the in vitro data while having the advantage of being relatively computationally inexpensive.

The stiffness properties defined by equation (1) for each rotational mode were scaled to each vertebral disc by applying a scale factor to the abscissa $(\theta)$ according to the percentage contribution of motion at each motion segment unit level relative to the overall global motion, as previously reported by White and Panjabi (1990). The resulting vertebral disc rotational stiffness parameters were lumped parameters, since their initial determination was derived from in vitro cadaveric experimentation using motion segments with surrounding ligaments and facet joints intact. Further scaling of the disc rotational stiffness was required, as additional tissue structures were incorporated into the model and is subsequently described in section 2.3.2.1. 

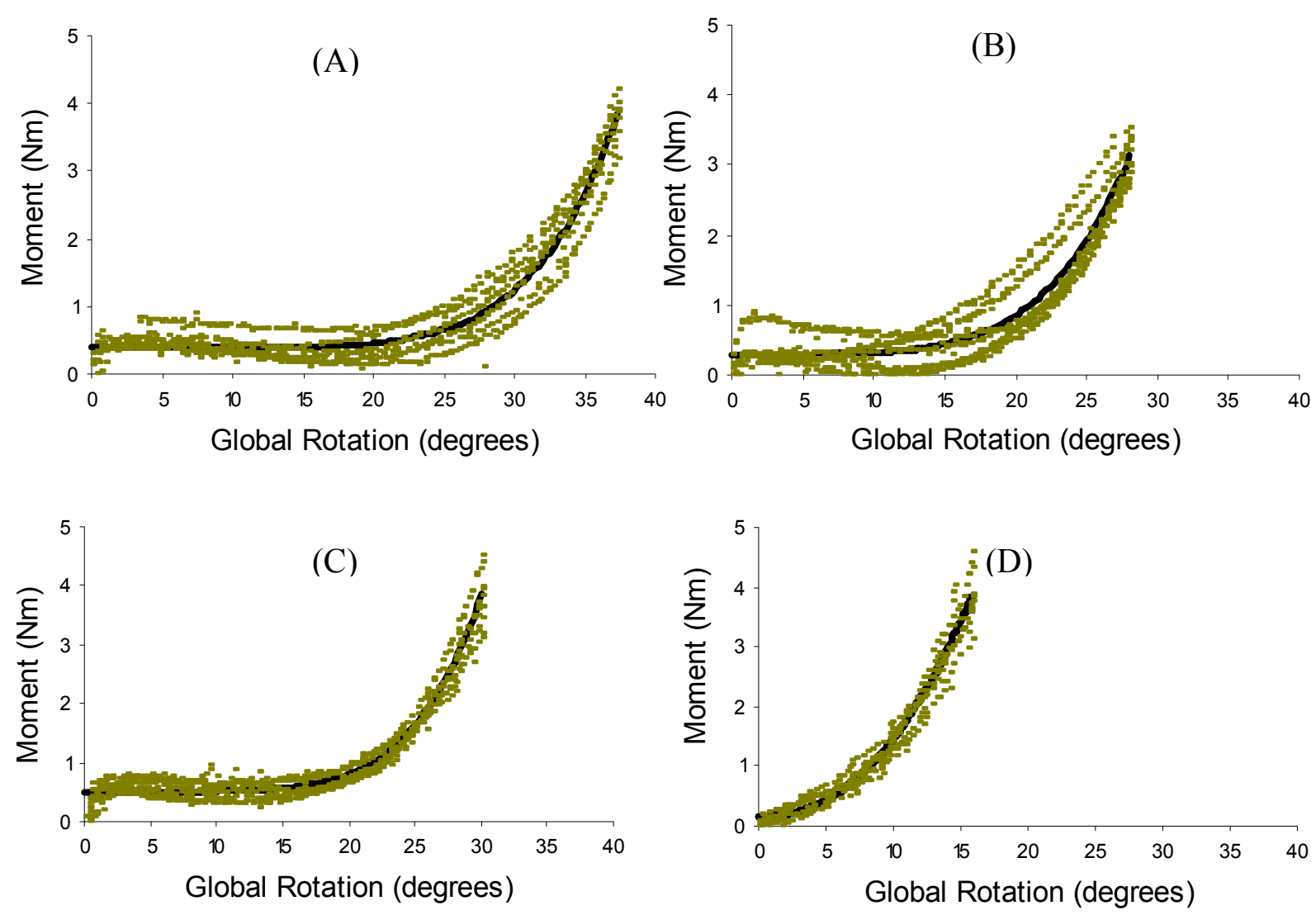

Figure 2.4 Averaged moment-rotation data (C2-T1) from six specimens. (A) Flexion, (B) Extension, (C) Lateral bending, and (D) Axial rotation. $\mathrm{R}^{2}$ values for curve fits were $0.91,0.87$, 0.95 , and 0.96 , respectively.

Table 2.2 Coefficients for global rotational stiffness properties (C2-T1).

\begin{tabular}{cccc}
\hline \multirow{2}{*}{ Rotation mode } & \multicolumn{3}{c}{ Constant coefficients } \\
\cline { 2 - 4 } & $a$ & $b$ & $c$ \\
\hline Flexion & 0.387 & $2.743 \times 10^{-10}$ & 6.424 \\
Extension & 0.301 & $2.945 \times 10^{-7}$ & 4.823 \\
Lateral bending & 0.506 & $5.707 \times 10^{-9}$ & 5.934 \\
Axial rotation & 0.162 & $6.791 \times 10^{-3}$ & 2.281 \\
\hline
\end{tabular}

Note: Boundary conditions $(\theta)$ are flexion $\left(0^{\circ}-37^{\circ}\right)$, extension $\left(0^{\circ}-28^{\circ}\right)$, lateral bending $\left(0^{\circ}-\right.$ $\left.30^{\circ}\right)$, and axial rotation $\left(0^{\circ}-16^{\circ}\right)$. 


\subsubsection{Facet joints}

The incline of the facet joints in the sagittal plane was determined by measurement on the bony surface of the facet joint of the reconstructed model (Table 2.1). Generally the angle of incline of the facet joints in the sagittal plane increases cephalocaudally. This finding was in agreement with the description of White and Panjabi (1990).

The facet joints of the cervical spine were modeled to rotate freely and translate along the $\mathrm{X}_{\mathrm{f}}$, $\mathrm{Y}_{\mathrm{f}}$, and $\mathrm{Z}_{\mathrm{f}}$ axes (Figure 2.2). In the sub-axial cervical region (C2 through $\mathrm{T} 1$ ), three orthogonal planes defined relative facet joint motion: 1) the sagittal plane, 2) the plane of the facet joint and 3) a third plane orthogonal to the other two planes (Bogduk and Mercer, 2000).

Anterior/posterior rotation occurred within the sagittal plane, while axial facets rotation occurred in the plane of the facet joint. Rotation in the third plane was limited by the directly opposed facets. Since the facet joint was a synovial joint, friction was assumed to be zero with shear translations along the $\mathrm{X}_{\mathrm{f}}$ and $\mathrm{Y}_{\mathrm{f}}$ axes. Translations along the facet $\mathrm{Z}_{\mathrm{f}}$ axis (tension and compression) were controlled by force-displacement relationships. Since no data describing these relationships for the facet joint could be found in the literature, data derived from similar tissues were used in place. Intervertebral disc stiffness data from Shea et al. (1991) was used to define compressive stiffness while capsular ligament data from Yoganandan et al. (2001) was used to define the tensile stiffness. 


\subsubsection{Ligaments}

Ligaments were modeled as non-linear spring-damper elements that actively resisted tension but were passive in compression. To simulate ligament width, three parallel spring-damper elements were used for each of the anterior longitudinal ligaments (ALL), the posterior longitudinal ligaments (PLL), and the ligamentum flavum (LF). The width of each ligament (i.e., the maximum distance between parallel spring-damper elements) were derived from Panjabi et al. (1991). Interspinous ligaments (ISL) and supraspinous ligaments (SSL) were modeled as single spring-damper elements. Since ligaments span over several vertebrae, it was not possible to set the origins and insertions of ligaments at the exact anatomic locations. In this model the origins and insertions of ligaments were assumed to be at the margin of the vertebrae.

The viscous behavior for all ligaments was defined by a constant damping coefficient 'c' with a value of $0.4 \mathrm{Ns} / \mathrm{mm}$, which was adapted from van der Horst (2002) and Yoganandan et al. (1989). The non-linear elastic behavior of each ligament was governed by stiffness (k) - strain (e) curves (Figure 2.5), which were generated using data from the literature (Yoganandan et al., 2000; 2001).

Since the ALL, PLL, and LF were modeled as three parallel spring-damper elements, the actual stiffness values used for each individual spring were scaled to $1 / 3$. The stiffness for ISL 


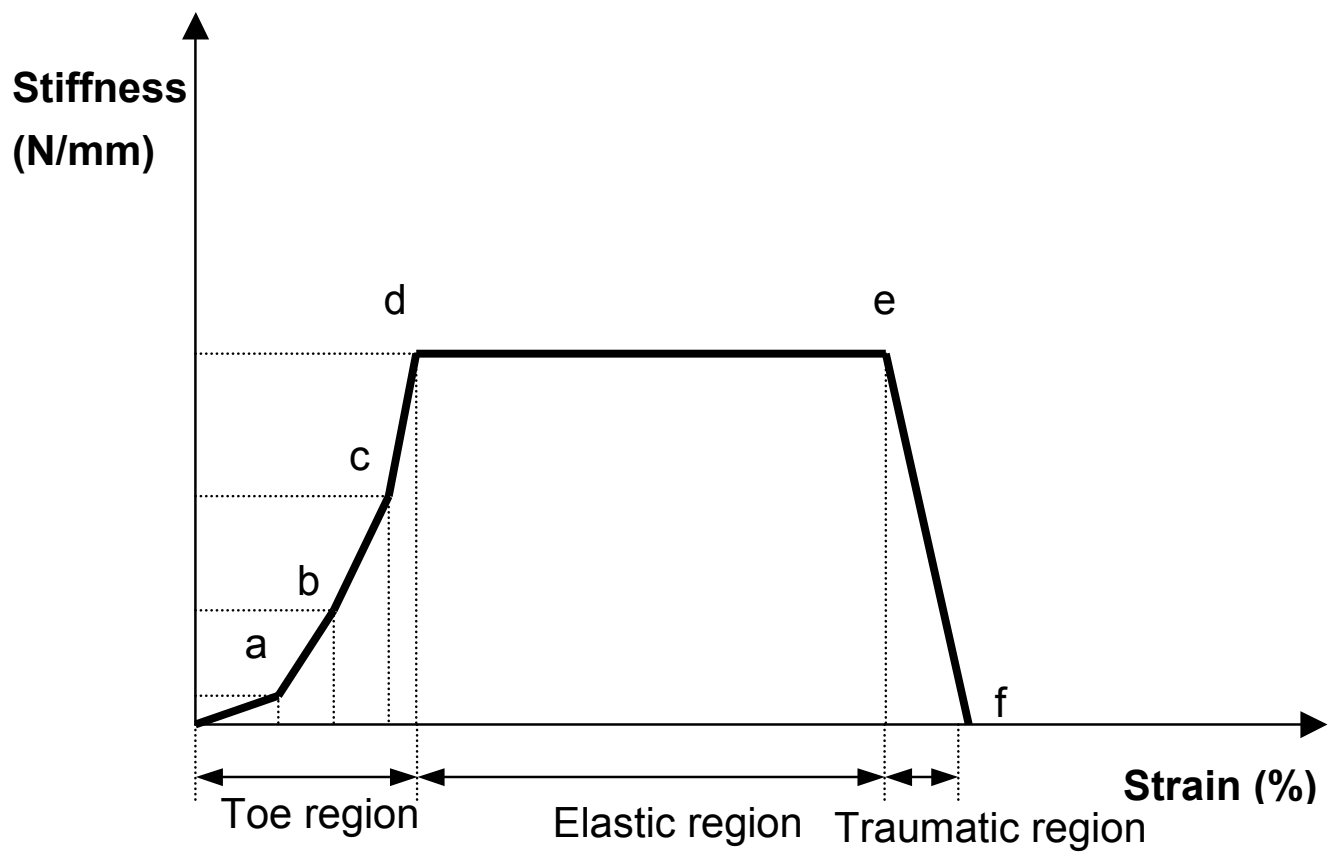

Figure 2.5 The stiffness (k) vs. strain (e) response of a ligament. Points ' $a$ ', 'b', and 'c' were defined at $5 \%, 10 \%$, and $15 \%$ of failure strain and $6.25 \%, 25 \%$, and $50 \%$ of stiffness respectively. Points ' $d$ ' and point 'e' were assumed at $20 \%$ and $90 \%$ of the failure strain (point ' $\mathrm{f}$ '), respectively. Graph is not to scale. 
and SSL were scaled to $1 / 3$ and 2/3 respectively, based on data from the literature (Yoganandan et al., 2000).

\subsubsection{Modeling of virtual laboratory simulator}

We have previously developed an in vitro experimental protocol that induced a bending moment distribution across the cervical spine which resulted in a motion response that more closely matches the in vivo case (DiAngelo and Foley, 2003; 2004; Foley et al., 1999). The virtual experimental protocol here-in replicated our in vitro protocol and is illustrated in Figure 2.6. The test apparatus consisted of an upper fixture housing a linear bearing and splined shaft assembly mounted in a rotating joint that attached to a vertical actuator. For flexion, extension, and lateral bending test, the cervical spine was placed in an inverted neutral orientation eccentric to the load axis of the actuator. Downward motion of the actuator shaft induced a compressive load and bending moment on the upper mounting pot (Figure 2.6). For axial rotation, the cervical spine was placed in an upright neutral position. A revolute motor located at the center of the lower mounting pot provided axial rotation. The upper mounting pot was unconstrained in the vertical direction (Tz) (Figure 2.6). Simulations of the virtual experimentation in VisualNastran 4D were performed using the Kutta-Merson integration $\left(5^{\text {th }}\right.$ order $)$ with variable 
(A)

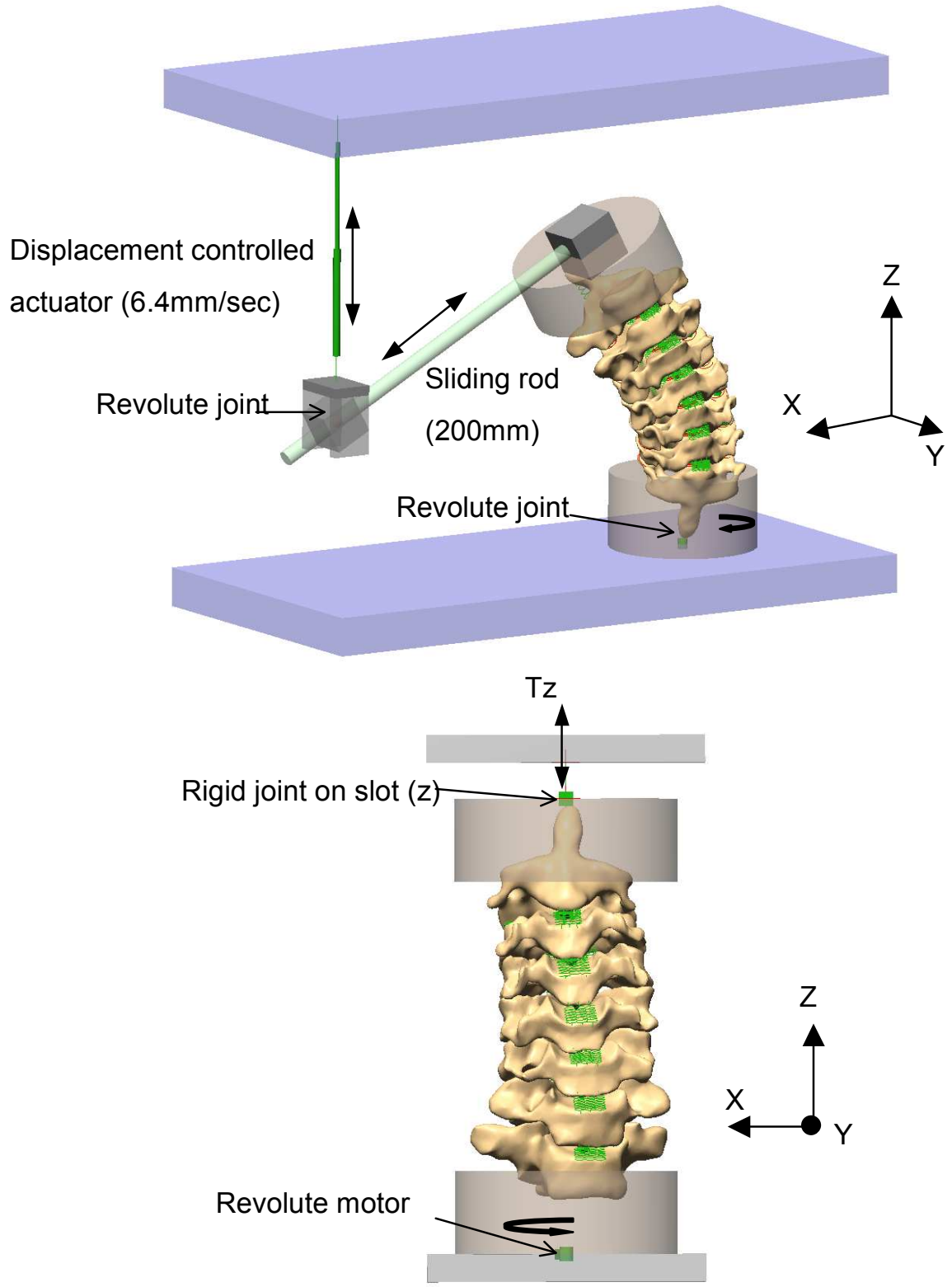

Figure 2.6 The virtual experimentation set-up. (A) Lateral bending loading condition: this set-up was also used for flexion and extension loading conditions. (B) Axial rotation loading condition. The important parameters of the experimental set-up (the initial moment arm: $200 \mathrm{~mm}$, and the speed of actuator: $6.4 \mathrm{~mm} / \mathrm{sec}$ ) were adapted from the parameters of our experimental testing protocol. The final range of rotation for flexion, extension, lateral bending, and axial rotation test were $37^{\circ}, 28^{\circ}, 30^{\circ}$, and $16^{\circ}$, respectively. 
time step. The position tolerance and the orientation tolerance were $0.05 \mathrm{~mm}$ and $0.05^{\circ}$,

respectively.

\subsubsection{Method of model evaluation}

The behavior of the model during simulated experimental testing was evaluated both kinematically and kinetically. In the kinematic evaluation, motion segment unit rotations, motion coupling effects, and three-dimensional helical axes of motion (HAM) between adjacent vertebrae were compared to data obtained from our in vitro testing protocol and from the literature (White and Panjabi, 1990; Panjabi et al., 2001; Amevo et al., 1991; Milne, 1993). The computation of HAM parameters was performed in a LabVIEW routine (National Instruments, Austin, TX), which was developed in our Biomechanics Laboratory using the method described by Beggs (1983). The HAMs were computed for motion intervals starting from an initial vertebral body position to another vertebral body position every 0.01 seconds. Therefore, the last computed and animated HAM represented the complete motion history of each motion segment unit. The simulation run times for full flexion, extension, lateral bending, and axial rotations were $17,17,15$, and 7 seconds, respectively.

For kinetic evaluation, the global stiffness curves (C2-T1) for each loading condition were compared to global stiffness curves derived from our in vitro experimental protocol (Schwab, 
2003). In addition, the local intervertebral disc joint and facet joint load vectors across each segment were calculated and visually animated during the simulation to observe the individual level response to a globally applied external load.

\subsection{Evaluation of the virtual model}

\subsubsection{Kinematic evaluation}

\subsubsection{Relative motion segment unit rotations}

The contribution of each motion segment unit relative to the overall global motion of cervical spine was compared between the virtual model, our in vitro testing protocol (DiAngelo and Foley, 2004), and published in vivo data (White and Panjabi, 1990) for combined flexionextension, one sided lateral bending, and one sided axial rotation (Figure 2.7). The simulation results of the individual motion segment contribution to global rotation were comparable to the in vivo data.

\subsubsection{Coupling effect}

Using rotational data obtained from Panjabi et al. (2001), the motion coupling ratios at each vertebral level were computed for full ranges of global axial and lateral movements and compared with the virtual model. The lateral bending ratio was defined as the ratio of axial 
(A)

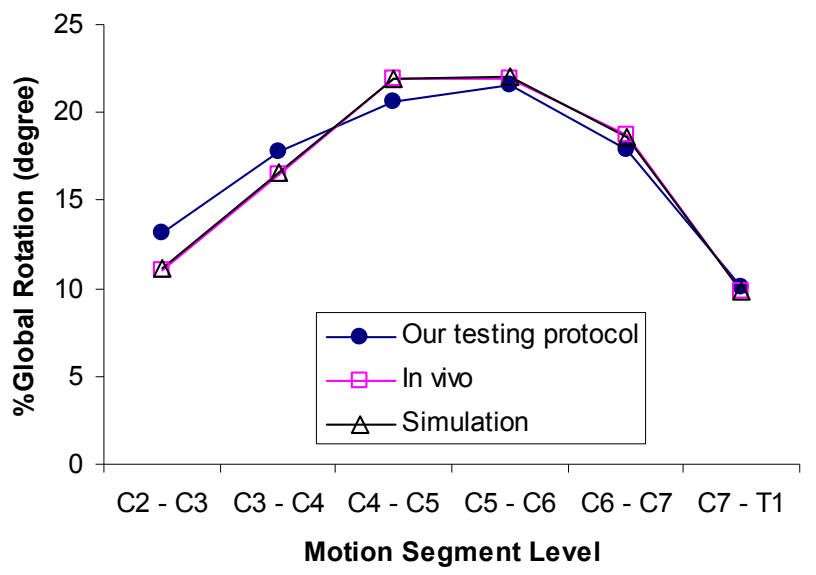

(B)
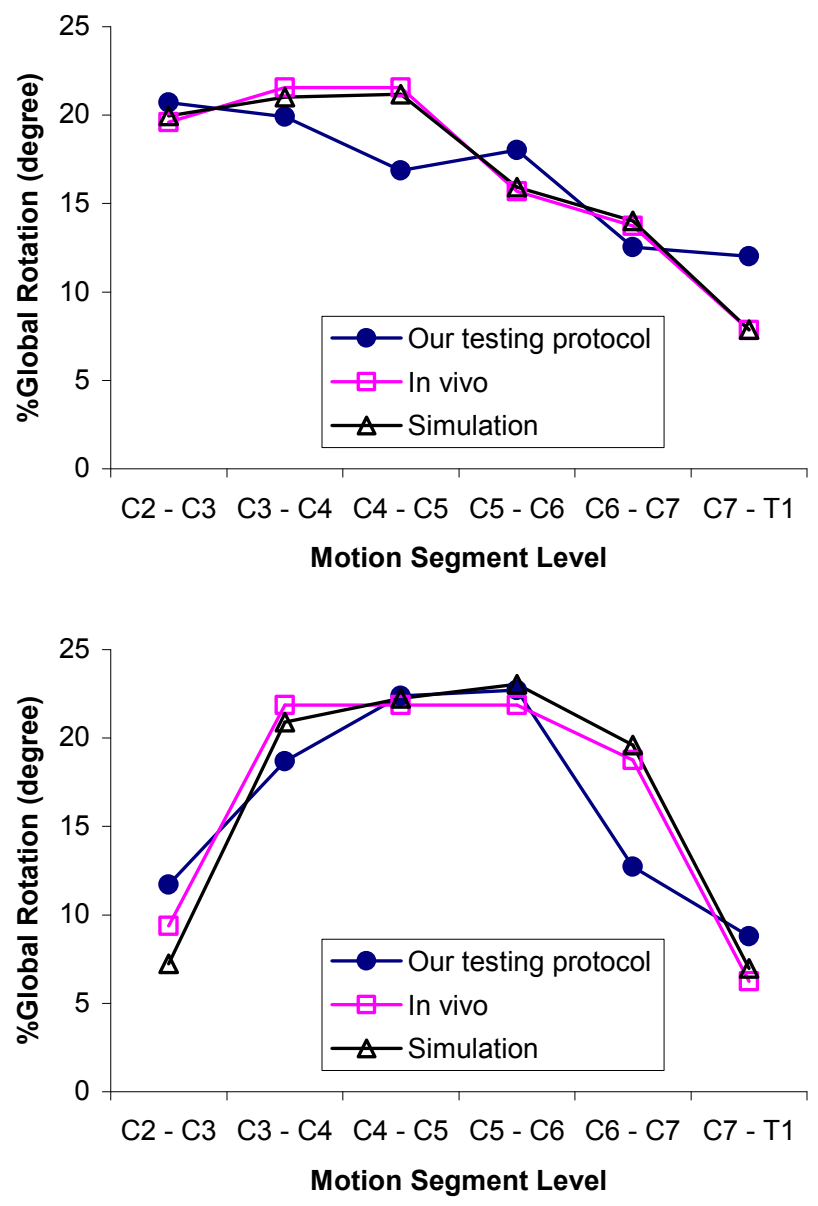

Figure 2.7 The comparisons of the distribution of relative motion changes: between our testing protocol, in vivo, and simulation. (A) Combined flexion/extension, (B) One side lateral bending, and (C) One side axial rotation. The individual motion segment unit rotations were expressed as percentages of overall global rotation. 
rotation to lateral rotation, while the axial coupling ratio was defined as the inverse of the lateral bending ratio.

During lateral bending, the coupling responses at the upper segment levels $(\mathrm{C} 2-\mathrm{C} 3, \mathrm{C} 3-\mathrm{C} 4$, and C4-C6) were comparable to Panjabi et al. (2001) (Figure 2.8). Simulation of our existing in vitro axial testing protocol demonstrated a large deviation from the physiologic response, where the C2-C3 segment tilted with opposite direction (Figure 2.8).

\subsubsection{Three-dimensional HAMs}

Virtual animations of computed HAMs for the flexion, extension, lateral bending, and axial rotation moments for individual motion segment units were performed. During flexion and extension, the average location of the HAM vectors were close to the instantaneous axes of rotation (IARs) in normal volunteers (Amevo et al., 1991) (Figure 2.9). During lateral bending motion, the HAMs passed obliquely through the upper cervical vertebrae in an upward and posterior direction. This pattern agreed with the results from Penning and Wilmink (1987) (Figure 2.9). For axial rotation motion, HAMs for our experimental set-up (i.e., 1DOF end constraint allowing only vertical translation, Tz) did not produce a physiologic motion response. In simulation, the HAM between $\mathrm{C} 2$ and $\mathrm{C} 3$ lay in the transverse plane pointing in the anterior and posterior direction, indicating the main motion was lateral tilt. For the segments $(\mathrm{C} 3-\mathrm{C} 4$ and 
(A)

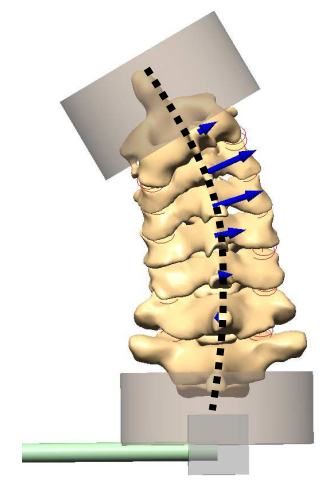

(B)

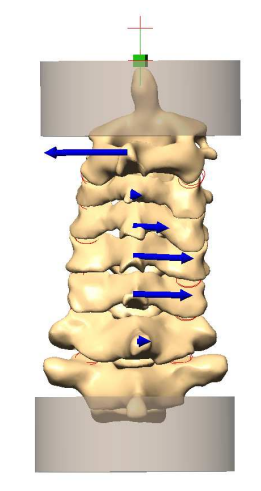

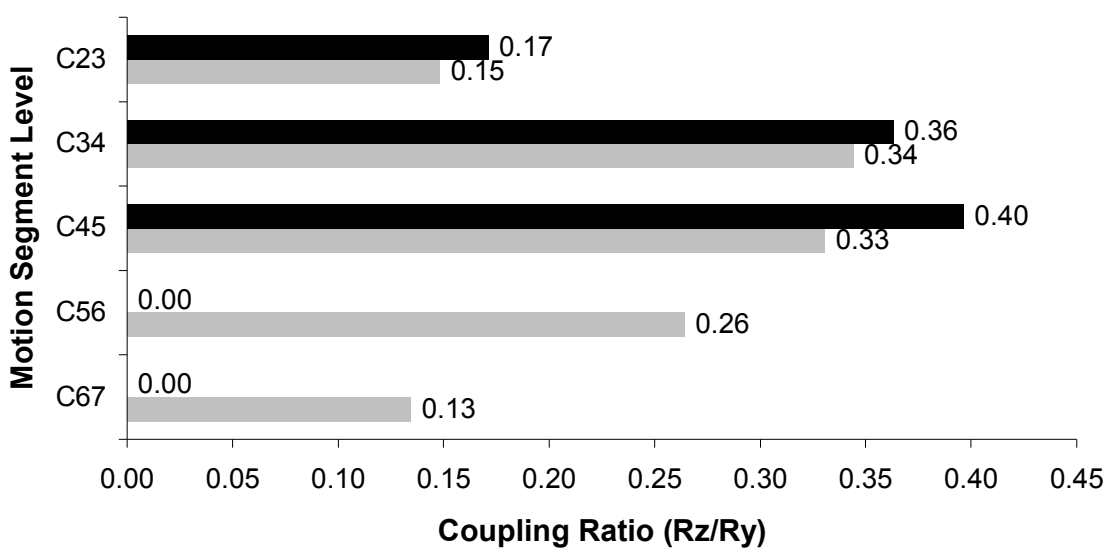

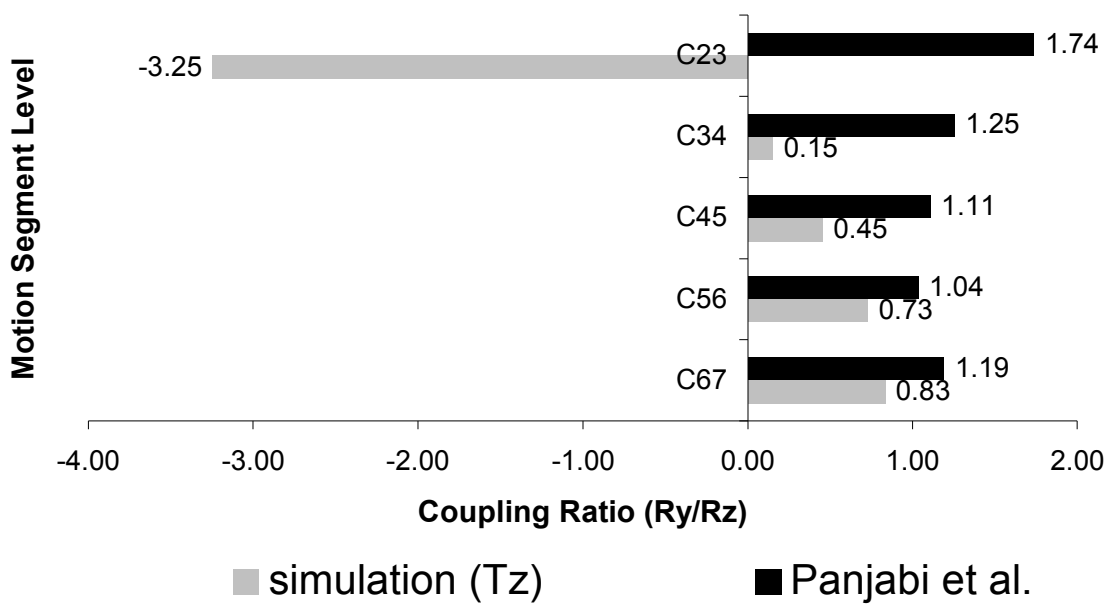

Figure 2.8 Coupled behaviors between lateral bending and axial rotation. (A) lateral bending and (B) Axial rotation. The blue arrows illustrate direction of coupled axial rotation and lateral bending. The length of the arrows represents the magnitude of the coupling. During lateral bending, the coupling ratio of upper three motion segments (C2-C3, C3-C4, and C4-C5) were similar to those of Panjabi et al. (2001). During axial rotation, the simulation showed nonphysiologic coupling ratios, especially at the $\mathrm{C} 2-\mathrm{C} 3$ segment that tilted in the opposite direction to the direction of axial rotation. 
(A)

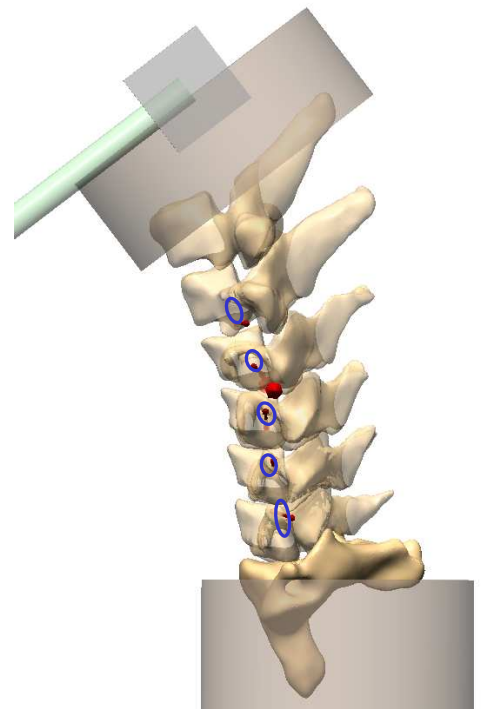

(C)

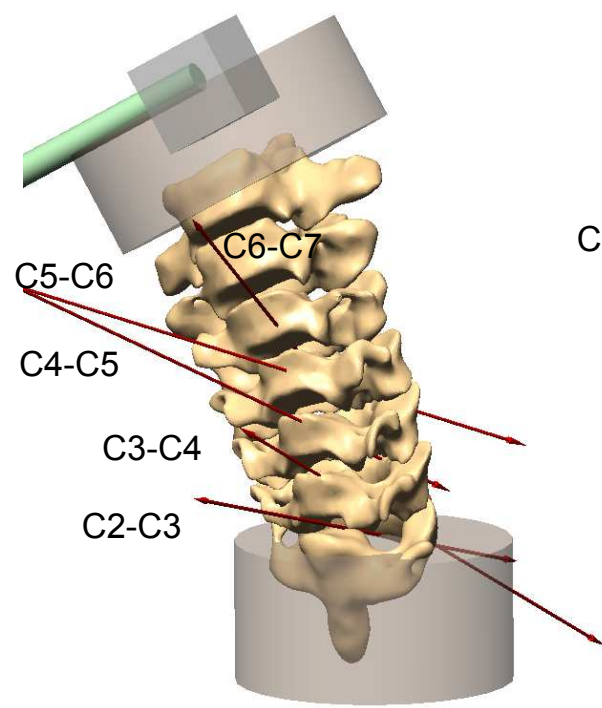

(B)

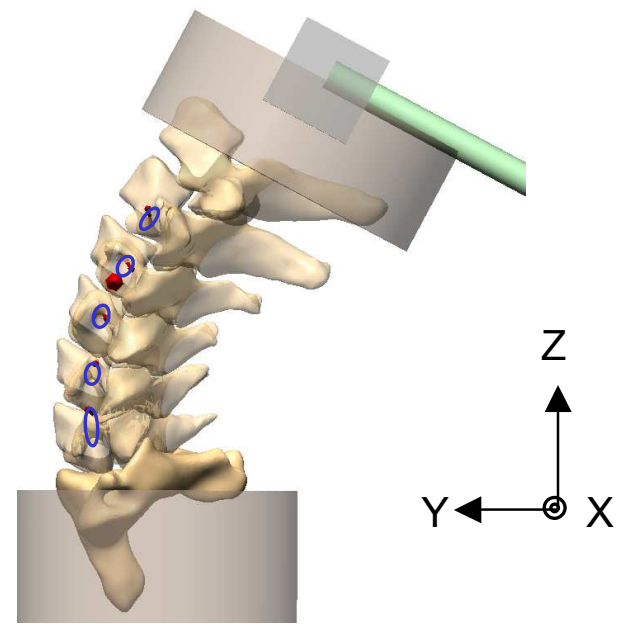

(D)

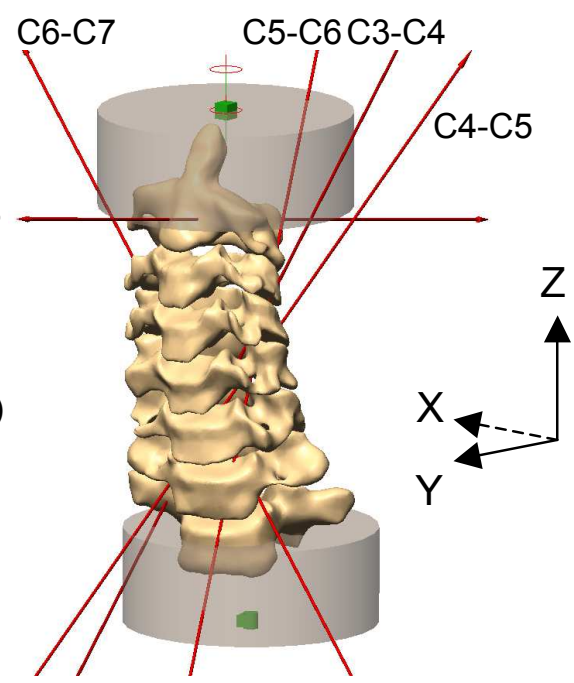

Figure 2.9 Animation frames of HAMs during flexion, extension, lateral bending, and axial rotation loading. (A) Flexion, (B) Extension, (C) Lateral bending, and (D) Axial rotation. During flexion and extension loading, all HAMs lie in the subjacent vertebral bodies. From C6C7 segment to C2-C3 segment, the local HAM vectors migrated caudally. The blue circles represent the distribution of the instantaneous axes of rotation reported by Amevo et al. (1991). During lateral bending loading, the HAMs passed obliquely through the upper cervical vertebrae in upper and posterior direction. The angles with the facet joints planes were less than $90^{\circ}$. During axial rotation loading, the non-physiologic pattern of HAMs were seen. 
C4-C5), the HAMs were shifted from the motion segment unit, indicating large translations between vertebral bodies (Figure 2.9). The axis-disc angles during lateral bending and axial rotation loading were measured and compared to the data reported by Milne (1993) (Figure 2.10). Although the angles of inclination were smaller, the segmental pattern of inclination was similar during lateral bending. The segmental pattern of inclination during axial rotation differed from the data reported by Milne (1993).

\subsubsection{Kinetic evaluation}

\subsubsection{Global rotational stiffness}

Final adjustments of ligament (tensile) and disc (rotational) stiffness properties were performed using an iterative process that sought to match the model global stiffness response to that of our in vitro data set. The same, pooled rotational stiffness curves (Schwab, 2003) used to generate regression equation (1) for each mode of global motion were redefined using a higher order Fourier series best-fit curve. Simulated testing of the virtual model was conducted and the global stiffness response for each mode statistically compared to the in vitro based best-fit response. If the two responses were dissimilar, scaling adjustments were made to the coefficient ' $c$ ' in equation (1), as well as, to the ligament stiffness values. The virtual tests were then repeated with the new set of global stiffness curves generated. After repeated trials, the final 
(A)

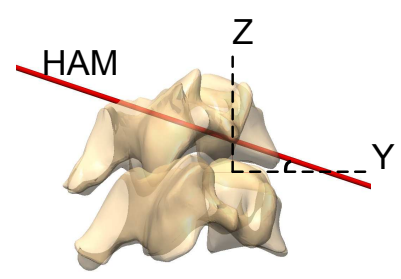

(B)

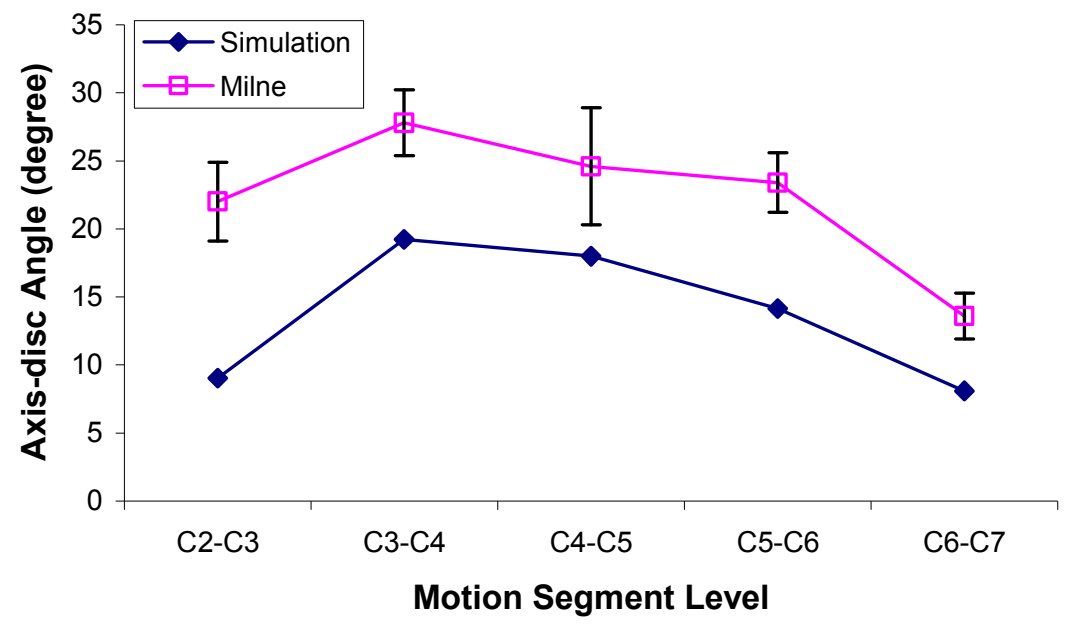

(C)

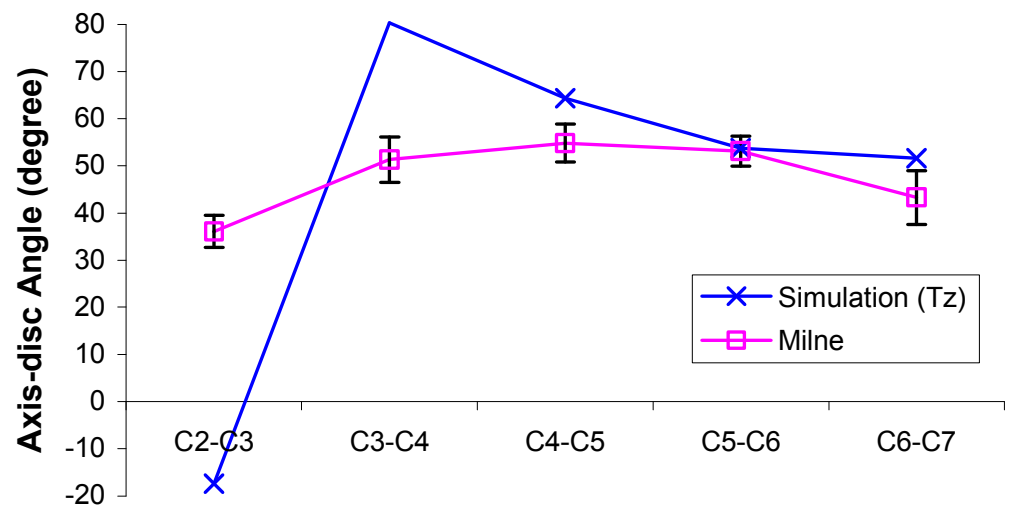

Motion Segment Level

Figure 2.10 Axis-disc angle during lateral bending and axial rotation loading. The data from Milne (1993) were represented with an average and one standard error bar. (A) Definition of axis-disc angle: the angle between a HAM and the axis $\mathrm{Y}$ in sagittal plane, (B) Axis-disc angle during lateral bending loading, and (C) Axis-disc angle during axial rotation loading. 
scale factors applied to the original coefficient ' $c$ ' values were $0.9875,1,0.97$, and 0.995 for

flexion, extension, lateral bending, and axial rotation, respectively. A final uniform scale factor of 0.525 was applied to ligament stiffness values for all modes.

Statistical comparisons between the virtual model and in vitro based best-fit stiffness curves were conducted using CurveAnalyzer 1.0 software (Chalmers University of Technology, Göteborg, Sweden) (Figure 2.11). The statistical tests included comparison of the means, coefficients of correlation (which compared the shape of the curves), and the standard deviation of the residuals (which measured the spread of the data) (Table 2.3). By applying the above scaling values to model parameters, all global stiffness curves generated by the simulation passed all of the statistical comparison tests.

\subsubsection{Local force vector analysis}

The force vectors at the intervertebral disc and facet joints were computed and animated during the virtual simulation. During flexion, the magnitude of the forces at each intervertebral disc joint gradually increased during the first $20^{\circ}$ of global flexion. At the final end limit of flexion $\left(37.315^{\circ}\right)$, the load at the intervertebral disc joints of the $\mathrm{C} 4-\mathrm{C} 5$ and $\mathrm{C} 5-\mathrm{C} 6$ motion segments was greatest (Figure 2.12). During flexion, the facet joints were under compression at upper motion segments (C2-C3, $\mathrm{C} 3-\mathrm{C} 4$, and $\mathrm{C} 4-\mathrm{C} 5)$ and under tension at the lower motion 

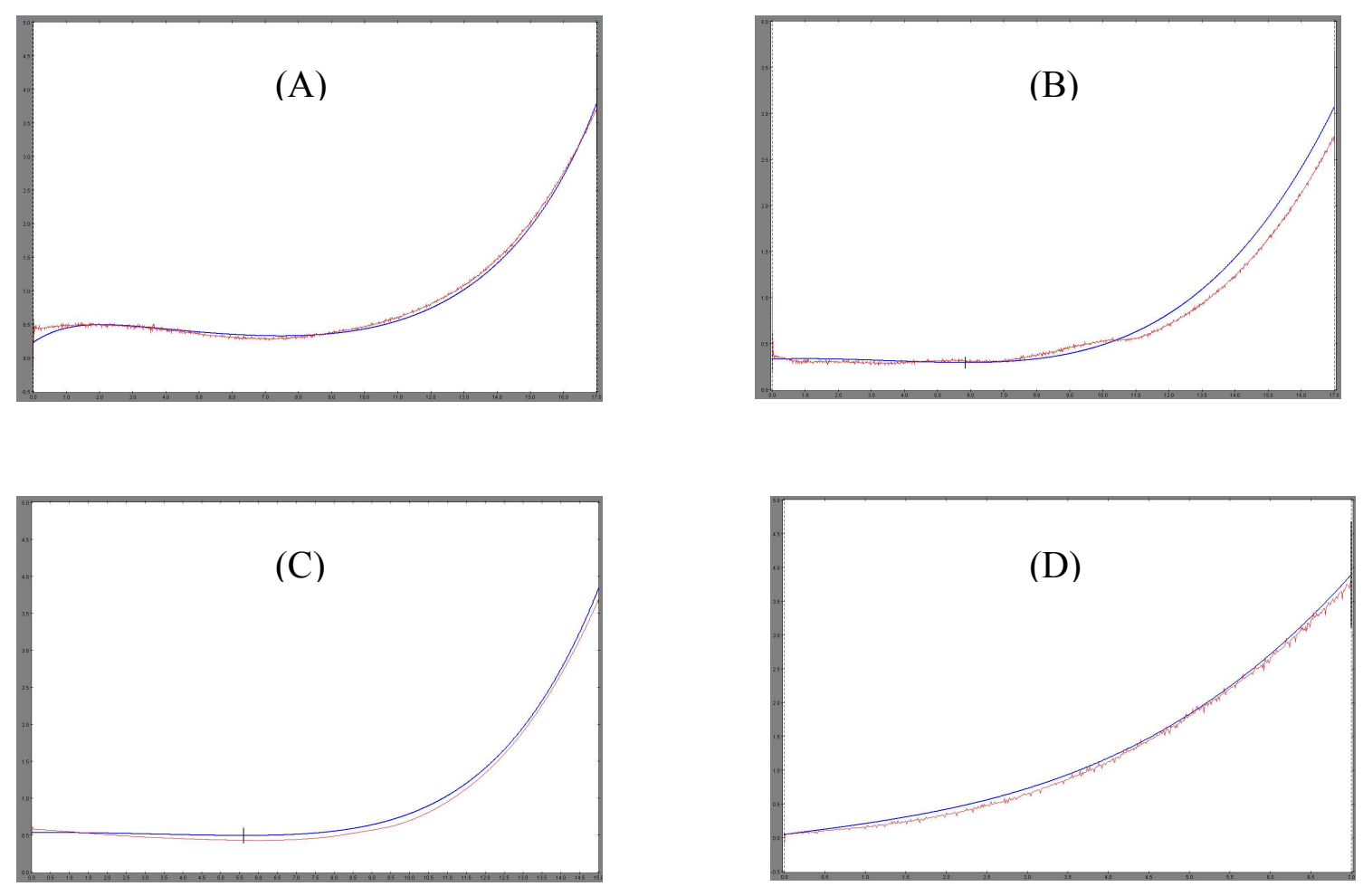

Figure 2.11 Kinetic evaluation of global rotational stiffness properties. (A) Flexion, (B) Extension, (C) Lateral bending, and (D) Axial rotation loading. Blue = representative curve, Red $=$ simulation curve

Table 2.3 Statistical evaluations of global stiffness curves.

\begin{tabular}{ccccccc}
\hline Test & Parameter & Value & Flexion & Extension & $\begin{array}{c}\text { Lateral } \\
\text { bending }\end{array}$ & $\begin{array}{c}\text { Axial } \\
\text { rotation }\end{array}$ \\
\hline Mean & L & 0.2 & $\boldsymbol{V}$ & $\boldsymbol{V}$ & $\boldsymbol{V}$ & $\boldsymbol{V}$ \\
Correlation & $\mathrm{r}$ & 0.8 & $\boldsymbol{V}$ & $\boldsymbol{V}$ & $\boldsymbol{V}$ & $\boldsymbol{V}$ \\
Std. residual & $\mathrm{S}$ & 0.2 & $\boldsymbol{V}$ & $\boldsymbol{V}$ & $\boldsymbol{V}$ & $\boldsymbol{V}$ \\
\hline
\end{tabular}

Note: $\boldsymbol{V}=$ satisfied, $\boldsymbol{X}=$ unsatisfied 

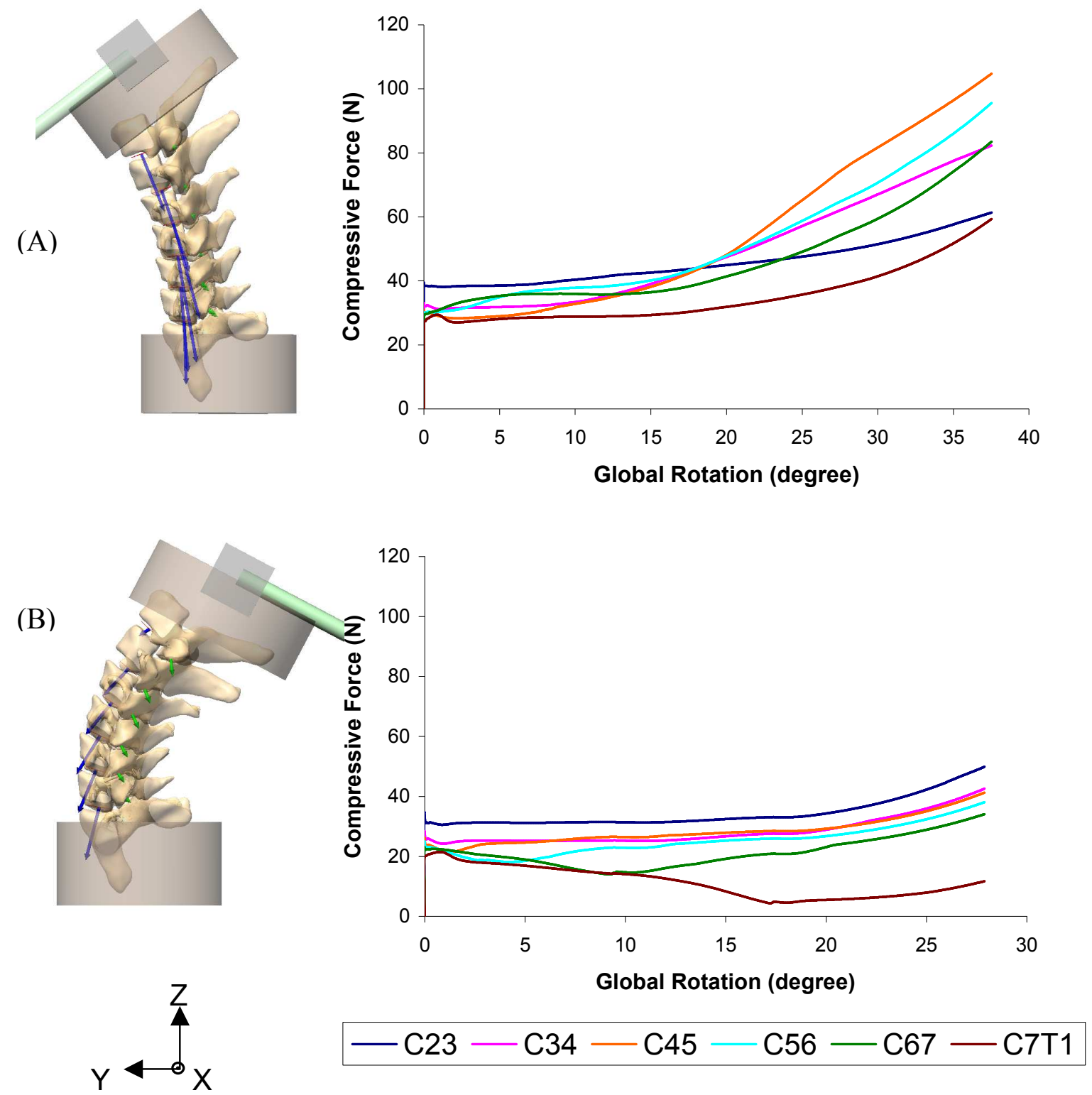

Figure 2.12 Animation frames of local forces and the plots of compressive forces versus global rotation during (A) flexion and (B) extension. Applied moment was $3.65 \mathrm{Nm}$ for flexion, $2.65 \mathrm{Nm}$ for extension. The arrows illustrate the force vectors: Blue arrow $=$ force vector of intervertebral disc joint; Green arrow $=$ force vector of facet joint. 
segments (C5-C6, C6-C7, and C7-T1). However, the magnitudes of the facet forces during flexion were small. The average compressive loads associated the activities of daily living in the cervical spine are estimated to be between 70 and 150N (Anderson and Rouleau, 2004). For the simulation study, the average compressive load (C2 to T1) at the terminal flexion was $81 \mathrm{~N}$. During extension, the magnitude of the forces at each intervertebral disc joint also increased gradually but in a different way. The lower segments, especially C7-T1, were initially under tension. However, by the end of extension $\left(28.037^{\circ}\right)$, the greatest load occurred at $\mathrm{C} 2-\mathrm{C} 3$, while smallest load occurred at C7-T1 (Figure 2.12). The facet joints in all of the motion segment units experienced compressive loading and held more load compared to those in flexion. The compressive force at the facet joints was greatest at $\mathrm{C} 7-\mathrm{T} 1$ and least at the $\mathrm{C} 2-\mathrm{C} 3$ (Figure 2.12). During left lateral bending, the intervertebral disc joint force experienced minimal changes after the initial equilibrium loading was achieved. However, the facet joint loads exhibited large increments during lateral bending motion. At the end limit of left lateral bending $\left(30.063^{\circ}\right)$, the left-side facet joints were under compression, while the right-side facet joints were under tension (Figure 2.13). During axial rotation, minimal change occurred at the intervertebral disc joint forces after the initial equilibrium loading was reached. By the end of axial rotation $\left(15.792^{\circ}\right)$, the force vectors at the upper motion segment levels (C2-C3, C3-C4, and C4-C5) angled 

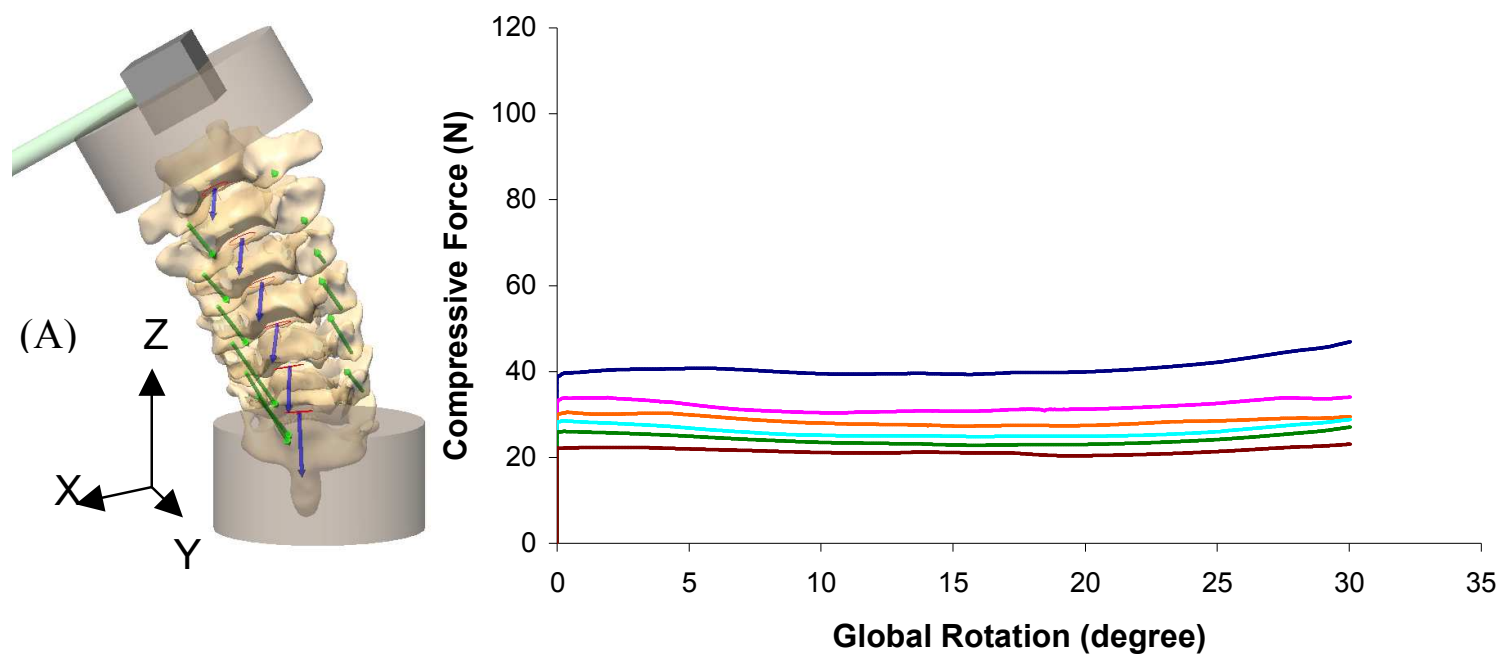

(B)
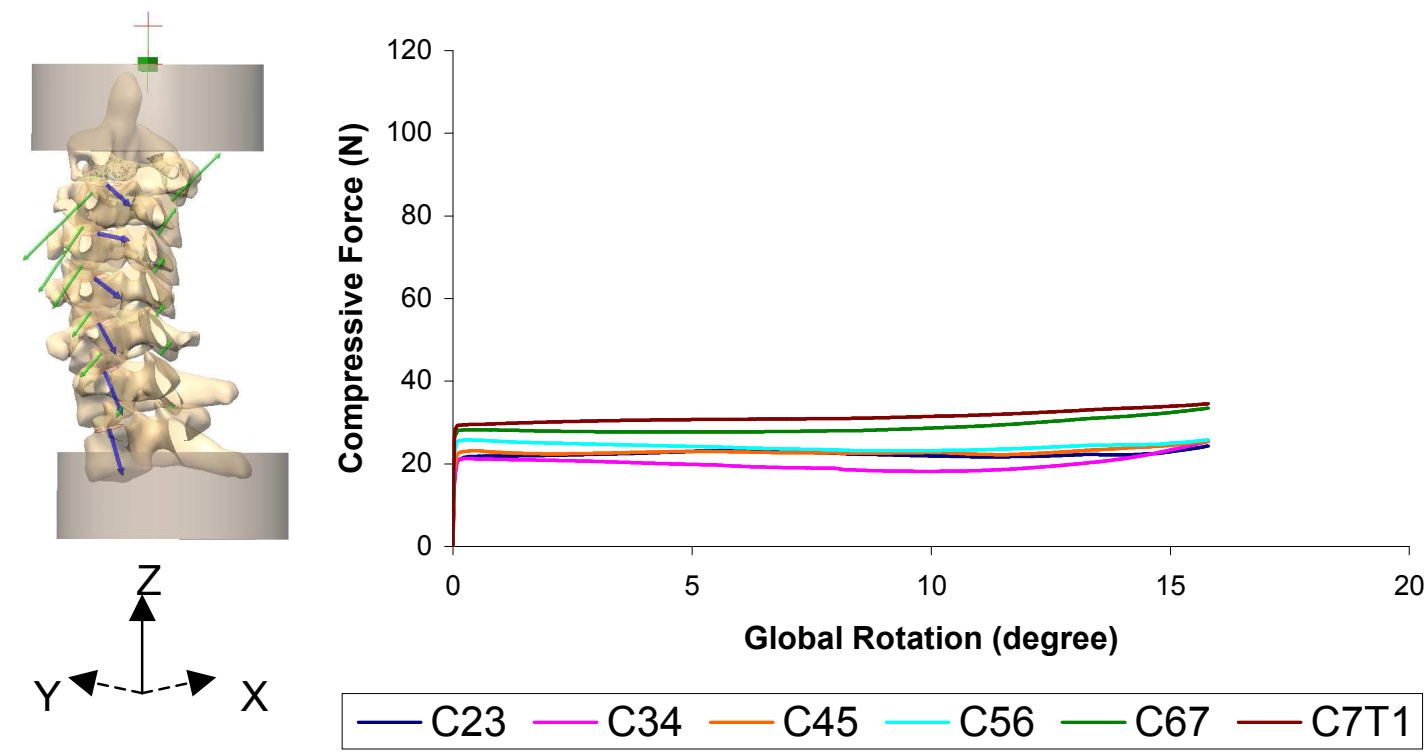

Figure 2.13 Animation frames of local forces and the plots of compressive forces versus global rotation during (A) lateral bending and (B) axial rotation. Applied moment was $3.61 \mathrm{Nm}$ for lateral bending and $3.80 \mathrm{Nm}$ for axial rotation. The arrows illustrate the force vectors: Blue arrow $=$ force vector of intervertebral disc joint; Green arrow $=$ force vector of facet joint. 
backward, indicating primarily shear force components. The facet joint forces also increased towards the end of the rotation, especially at the upper motion segments (Figure 2.13).

\subsection{Discussion}

\subsubsection{Flexion, extension, and lateral bending}

The distribution of the MSU rotations of the virtual model agreed well with the in vivo values from White and Panjabi (1990) and with our in vitro laboratory data. During lateral bending, there was concurrence between the motion coupling behavior of the virtual model with data from Panjabi et al. (2001) In that study, there was minimal amount of coupling detected at segments C5-C6 and C6-C7. However, in the simulation model, large amounts of coupling were detected. Although this ratio was high at segments $\mathrm{C} 5-\mathrm{C} 6$ and $\mathrm{C} 6-\mathrm{C} 7$, the actual amounts of axial rotation and lateral bending were small. In the computer simulation, these subtle changes could be detected, however, it is most likely impossible to detect this small change during in vitro testing.

HAM analysis provides a good method of model evaluation since it is highly unlikely that a combination of incorrect material properties for each structural element would accidentally produce realistic HAMs at each motion segment unit. The HAM vectors are also closely related to the applied loading condition. HAM vectors obtained during flexion and extension were 
comparable to the two-dimensional sagittal plane in vivo IAR data from Amevo et al. (1991).

The HAMs at each segment fell within two standard deviation ranges of interobserver

differences. Consequently, the virtual model properties and the applied flexion/extension

loading conditions in the experimental protocol reproduced the in vivo kinematic response. For

lateral bending, the HAMs were compared to the in vitro measurement from Milne (1993). The

HAMs were located a little superior in the moving vertebral bodies and the disc-axis angles

followed the same pattern with smaller amounts of inclination. The high incidence of HAMs in

the upper moving vertebral bodies could be explained by rocking movements about this axis

during lateral bending. These rocking movements have been well described in the literature

(Bogduk and Mercer, 2000) for cervical spine kinematics.

The global rotational stiffness during flexion initially increased and then decreased before the parabolic increment of the curve. This phenomenon can be explained by the initial curvature of spine (lordotic curvature) and the experimental set-up. At the start of each simulation, the virtual spine tended to bend in extension due to gravitational loading combined with initial lordosis.

Therefore, a sizable initial actuator load was required to initiate flexion. As the lordotic curvature reduced, the load required to continue flexing the spine decreased. During extension, the initial lordotic orientation of the spine induced a negative moment on the actuator load cell, 
i.e., the actuator was holding up the spine. After some initial movement, the tissue stiffness engaged and the applied moment switched to a positive sense.

Although the virtual model provides an absolute value for the disc and facet forces, the output response may be better used to characterize the relative changes between them. Further, real time animation of the disc and facet joint force vectors provided instantaneous feedback of these changes.

\subsubsection{Axial rotation}

The simulation results during axial rotation did not follow the in vivo behavior. This was attributed from kinematic constraints of the current axial testing protocol (i.e., 1 DOF end constraint allowing only vertical translation). During axial rotation, the orientation of cervical facet joints should induce some lateral tilting. Therefore, the axial testing protocol was modified to allow lateral tilting or lateral tilt with lateral translation. With these modifications the simulation results produced physiologic kinematic and kinetic behavior. Consequently, a new axial testing apparatus was designed that allowed lateral tilt and translation to occur simultaneously with axial rotation. Results of this new in vitro method, along with accompanying virtual model simulation results are being prepared for publication. 


\subsubsection{Limitations}

This simulation model is not without limitations. The vertebral body geometries are only representation of a single human subject and therefore do not represent the anatomy of the general population. Similarly, initial alignment of the cervical column in the model was primarily determined by the original alignment of the vertebral bodies obtained from the CT data, which may not reflect neutral alignment of the general population, or that achieved during in vitro evaluations. Other geometrical limitations included potential errors in the selection of ligament attachment points and in the selection of coordinate frame locations in the disc and facet regions where the mechanical properties of these structures were applied.

Stiffness properties of the intervertebral discs were derived primarily from lumped in vitro stiffness data, which included the contribution of surrounding tissues. Stiffness properties of the discs were also applied to a single connecting location between vertebrae rather than distributed over bony vertebral surfaces and no hysteresis components or failure parameters for the discs were specified.

The facet joints were modeled as mechanical connections at a single defined point in space with specific stiffness properties rather than a contact surface. Tensile and compressive stiffness properties assigned to these joints were derived from similar tissues, since data describing these 
properties for the facet joint were not available. No hysteresis components or failure parameters for the facet joints were specified.

It was assumed that the virtual experimental protocol exactly duplicated the in vitro methods used in our laboratory, however slight differences may have occurred. Since the primary goal of the virtual model was to simulate current in vitro experiments, no active muscle components were included. Lastly, no parametric sensitivity studies of the model parameters were conducted.

\subsubsection{Conclusions}

A virtual model of the sub-axial cervical spine was developed to simulate our experimental laboratory testing method and used a physics-based dynamic simulation engine. The model provided realistic visualization of our in vitro experimental protocol for kinematics and kinetics analysis of cervical spine biomechanics. Other applications of the virtual cervical spine model include refinements to testing protocols, simulation of spinal fusion, and arthroplasty mechanics. Currently the virtual model does not include any active muscle components, as the initial goal was to simulate our existing in vitro testing protocol. However, the model could be developed to include muscle components for in vivo motion simulation. 


\section{Chapter 3}

\section{An Improved Axial Rotational Testing Protocol for the Cervical Spine}

\subsection{Introduction}

The cervical spine exhibits complex, coupled motions and loading behaviors. Biomechanical

testing on human cadaveric tissue should attempt to replicate the complexity encountered in

these physiologic systems. For sagittal plane (flexion/extension) motion, we have developed an experimental protocol that induces a bending moment distribution across the cervical spine. This results in a motion response that more closely matches the in vivo case (DiAngelo and Foley, 2003; 2004; Foley et al., 1999). A non-physiologic motion response was observed with our existing axial rotational testing protocol that permitted only vertical translation.

The objective of this study was to analyze the effect of kinematic and kinetic response for three different end mounting configurations for axial rotational testing on the sub-axial cervical spine. To accomplish this objective, a two part investigation was undertaken. The first part consisted of a computer simulation study to gain a more comprehensive understanding and visualization of the complex kinematics and kinetics of the sub-axial cervical spine during axial 
rotation testing. The second part consisted of an in vitro study by designing a new testing system for axial rotation with the explicit purpose of evaluating the simulation results.

\subsection{Methods}

\subsubsection{Development of the virtual computer simulation model}

A previously validated graphics-oriented multi-body model of the sub-axial cervical spine (C2-T1) and a virtual laboratory simulator were used to simulate the different end constraints conditions for axial rotational testing. This simulation model was previously described in detail. In the simulation model, the specimen was mounted in an upright neutral position. A revolute motor located at the center of lower mounting pot provided axial rotation. Three end constraint conditions for the upper mounting pot were studied: 1) Type 1: vertical translation (Tz), 2) Type 2: vertical translation $(\mathrm{Tz})$ and lateral tilt (Ry), and 3) Type 3: vertical translation (Tz), lateral tilt (Ry), and lateral translation (Tx) (Figure 3.1).

\subsubsection{Modified in vitro axial testing protocol}

Seven fresh human cadaveric cervical (C2-T1) spines were procured, prepared and tested in axial rotation with three end conditions analogous to the simulated end conditions. There were 


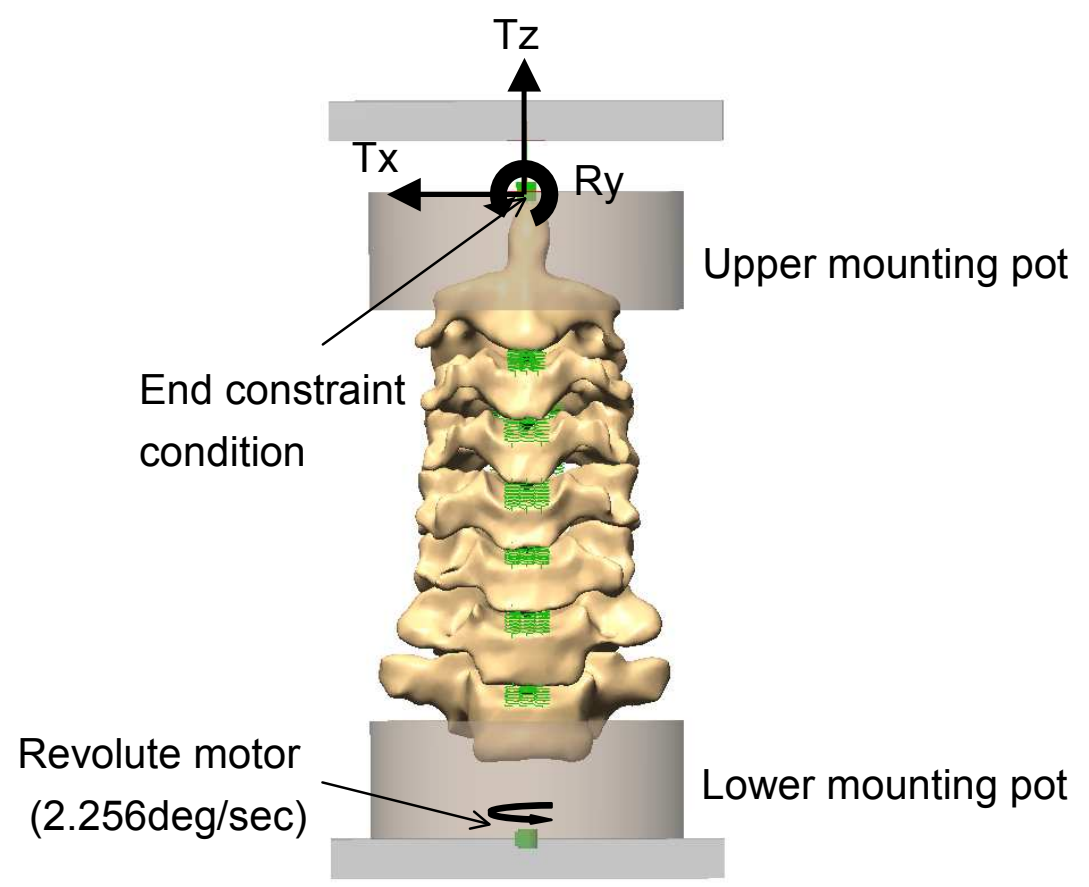

Figure 3.1 Schematic of the virtual experimental set-up for axial rotation tests. A revolute motor located at the center of lower mounting pot provided axial rotation and the upper mounting pot was unconstrained in three types of end constraint conditions. 
four female and three male specimens with an average age of $76 \pm 5$ years. The modified axial testing set-up is shown in Figure 3.2. The specimen was mounted in an inverted position to accommodate design limitations. Inverting the spine did not alter the mechanics or motion response. The upper mounting pot was unconstrained in the vertical direction $(\mathrm{Tz})$. The lower mounting pot was unconstrained in three types accordingly; 1) Type 1: no rotation nor translation was allowed at the lower pot (Tz), 2) Type 2: the lower mounting pot was unconstrained in lateral tilt $(\mathrm{Tz}+\mathrm{Ry})$, and 3) Type 3: the lower mounting pot was unconstrained in lateral tilt and lateral translation $(\mathrm{Tz}+\mathrm{Ry}+\mathrm{Tx})$ (Figure 3.2). Having the axial rotational input in the in vitro set-up applied to the $\mathrm{C} 2$ vertebra, as opposed to the $\mathrm{T} 1$ body in the simulation model, did not alter the loading or kinematic responses.

\subsection{Results}

The behavior of the virtual model was evaluated both kinematically and kinetically. In the kinematic evaluation, coupling behaviors, and three-dimensional helical axes of motion (HAM) were evaluated. For the kinetic evaluation, the global rotational stiffness curves (C2-T1) for different end constraints conditions were evaluated. In addition, the local intervertebral disc and facet joint load vectors of each motion segment unit (MSU) were visually animated in the 


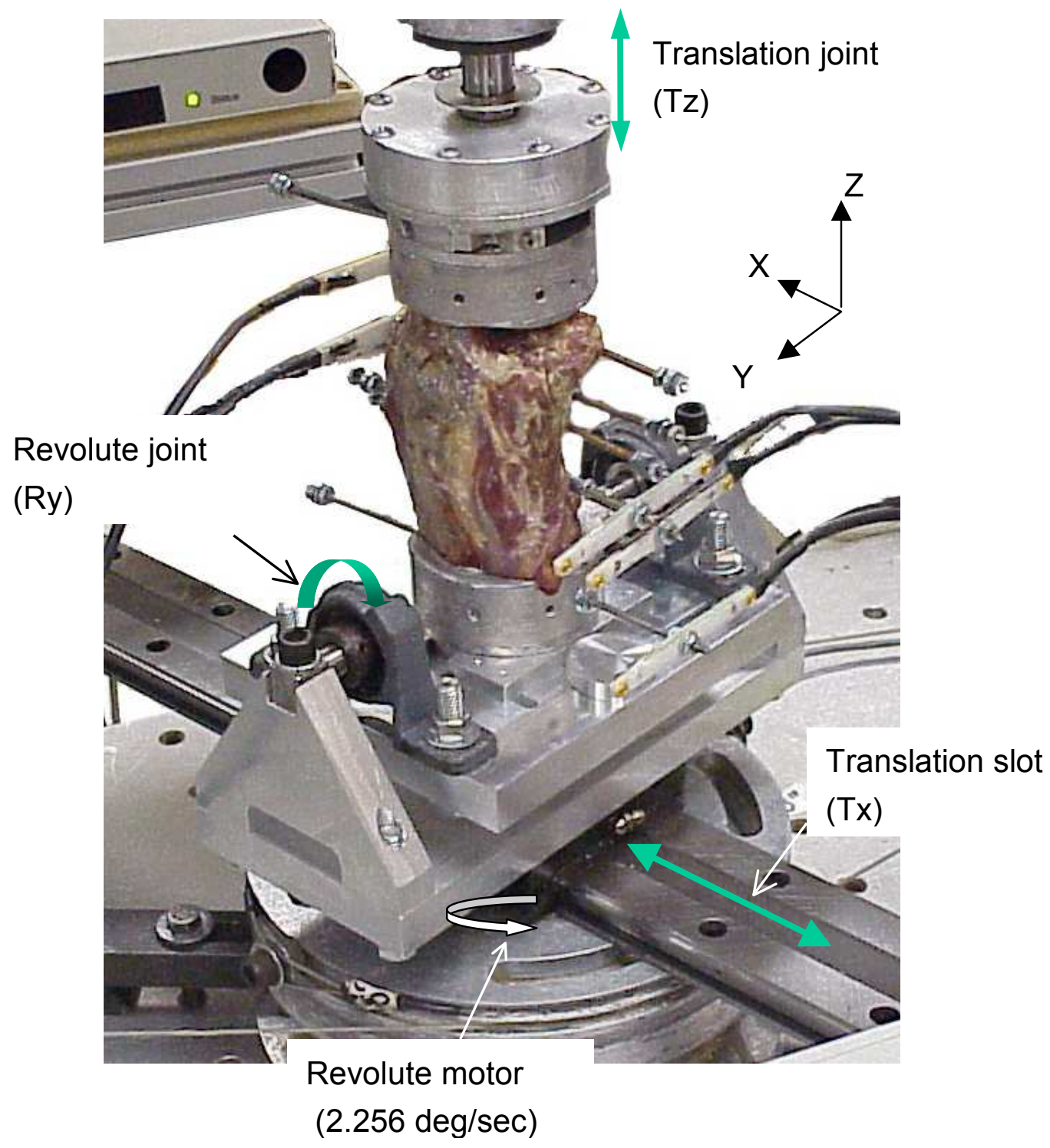

Figure 3.2 Modified testing set-up for in vitro axial rotation tests. The specimen was mounted in a neutral inverted upright position. Vertical translation (Tz) was allowed at the upper mount pot. Lateral tilt (Ry) and lateral translation (Tx) were allowed at the lower apparatus. 
simulation study to observe each MSU response to a globally applied external load. For the evaluation of the modified in vitro testing protocol, coupling behaviors and the global rotational stiffness curves were analyzed.

\subsubsection{Coupling behaviors}

The axial coupling ratio is defined as the ratio of lateral tilt to axial rotation. For the simulation model, the coupled motion responses of the different end mounting configurations are shown in Figure 3.3. Included in the comparison is the coupling ratio data from Panjabi et al. (2001) for full ranges of global axial movement (Figure 3.4). Rotationally constraining the spine (Type 1) resulted in significant lateral tilting of the C2-C3 MSU in a direction opposite to the axial rotation (Figure 3.3). Allowing rotation at the end mounts (Type 2 and Type 3), produced a coupling pattern that was in close concurrence with the physiologic response (Figure 3.3). The coupling ratio for Type 2 condition was closer to the coupling pattern from Panjabi et al. (2001) than the Type 3 condition (Figure 3.4).

A similar non-physiologic coupling response at the C2-C3 MSU occurred during the in vitro testing of the Type 1 condition, i.e., increased tilting of the C2-C3 MSU in the opposite direction to the axial rotation (Figure 3.4). Allowing some lateral rotation at the end mount (Type 2) resulted in a more physiologic response (Figure 3.4). Un-constraining the end mounts to 


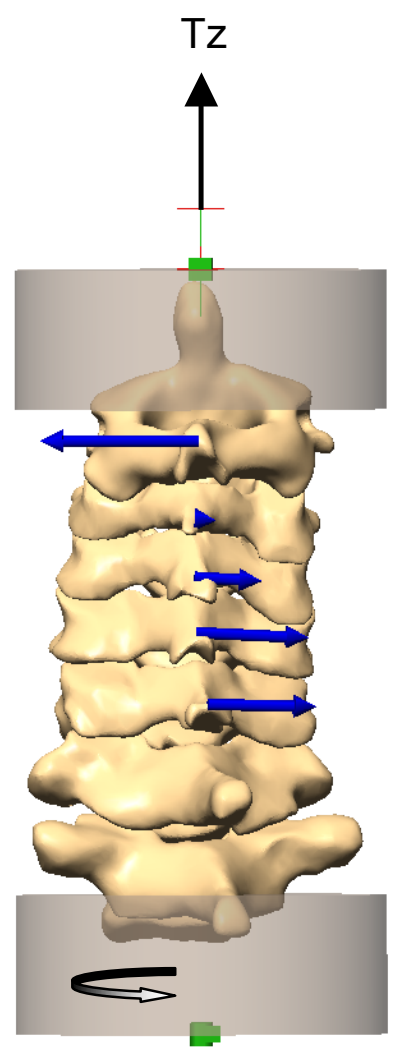

(A)

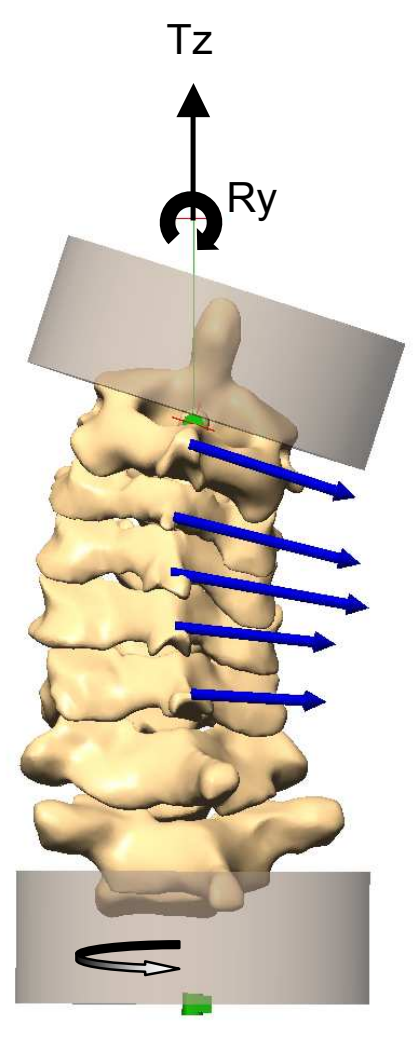

(B)

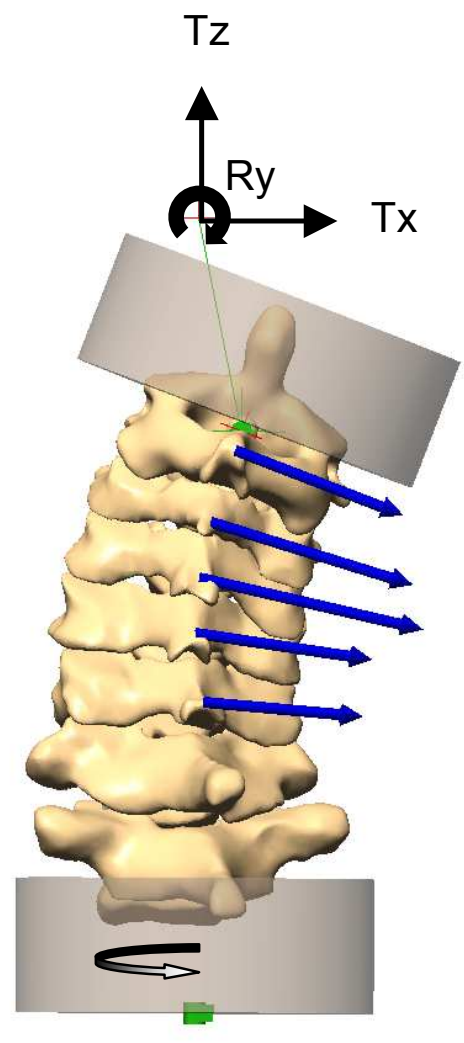

(C)

Figure 3.3 The coupling pattern between different end constraint conditions during axial rotation loading. (A) Type 1, (B) Type 2, and (C) Type 3 end constraint conditions. Thick arrows illustrate direction of coupled lateral bending. The length of the arrows represents the magnitude of coupling ratio. 


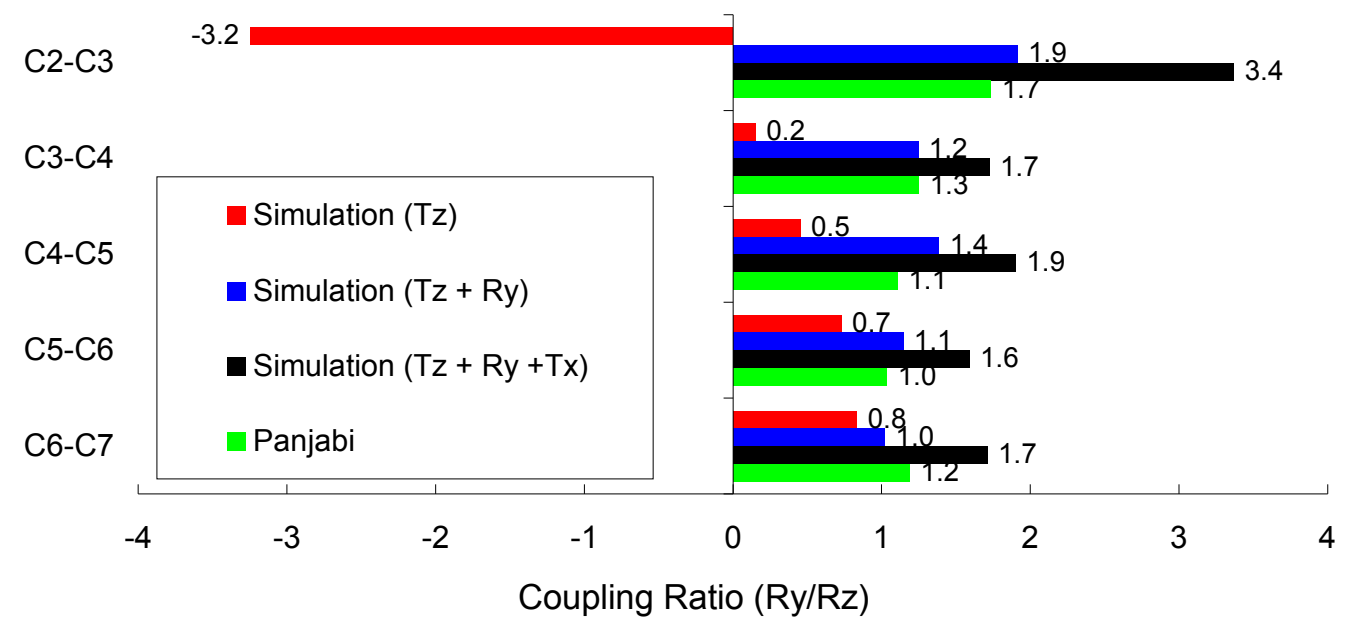

(A)

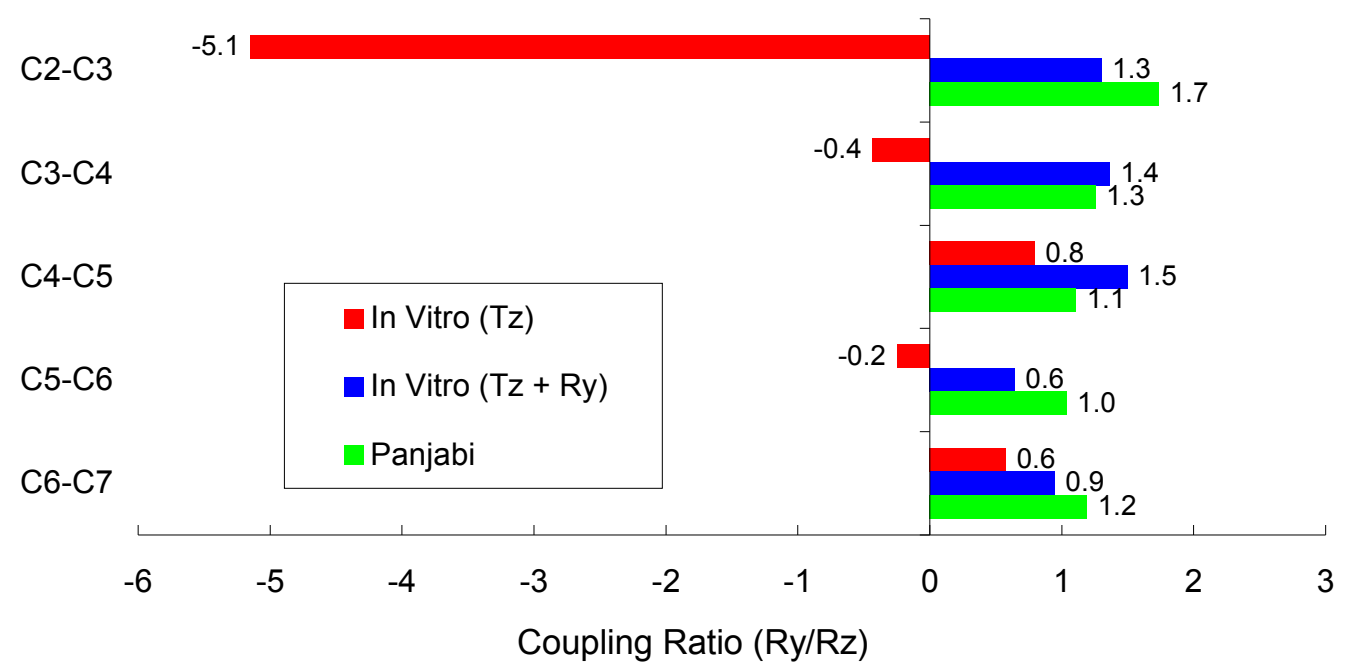

(B)

Figure 3.4 The coupling ratio plot. (A) Coupling ratio values from simulation study plotted against coupling ratio data from Panjabi et al. (2001). (B) Coupling ratio from in vitro tests plotted against coupling ratio data from Panjabi et al. (2001). 
translate freely in the lateral direction (Type 3) did not vary the motion response over the Type 2 condition.

\subsubsection{Helical axes of motion}

The simulation results of the HAM patterns of each MSU are shown in Figure 3.5 for the different end mount conditions. A non-physiologic response occurred at $\mathrm{C} 2-\mathrm{C} 3$ for the Type 1 end mount condition. The C2-C3 HAM vector laid in the horizontal plane directed in an anterior to posterior direction largely representative of a lateral tilting action. The HAMs of C3-C4 and C4-C5 were more vertically oriented (Figure 3.5). Un-constraining the upper pot to enable lateral rotation (Type 2) and translation (Type 3) produced a more physiological pattern of the HAM vectors, passing obliquely upward and backward (Figure 3.5). With the Type 2 condition, the HAM was located higher in the moving vertebral bodies compared to the location of HAM with Type 3 condition.

\subsubsection{Global rotational stiffness curves}

The global rotational stiffness curves from simulation and in vitro tests are shown in Figure

3.6. Allowing more degrees of freedom at the end mounts resulted in a notable decrease in rotational stiffness. In the simulation model, the global rotational stiffness was greatest with the 

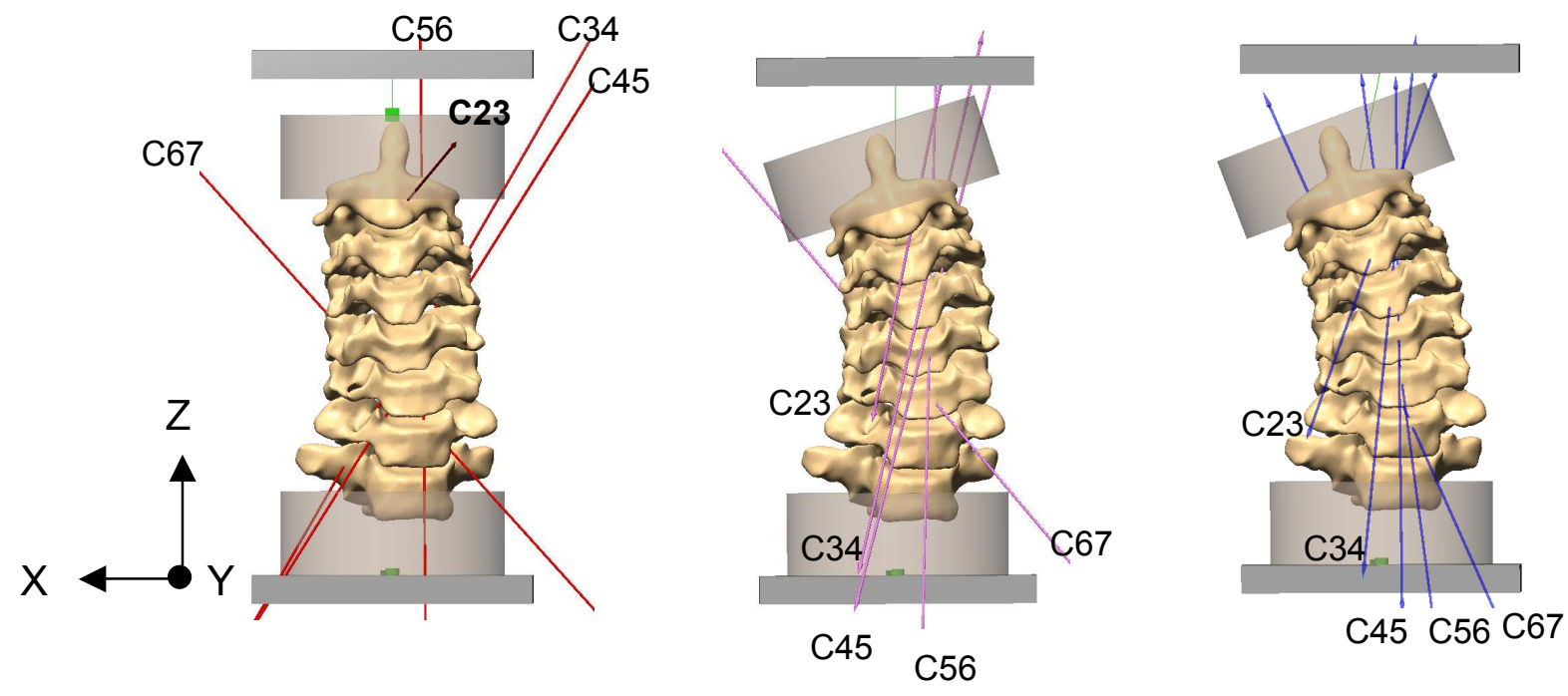

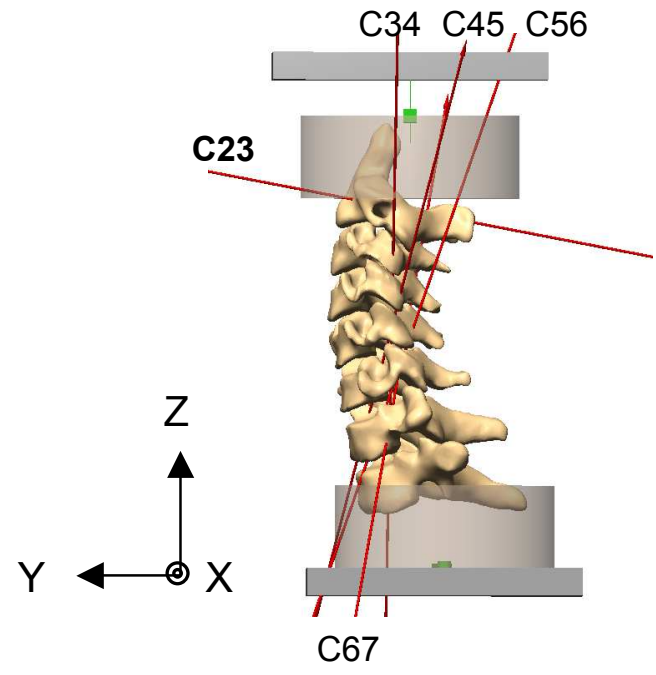

(A)

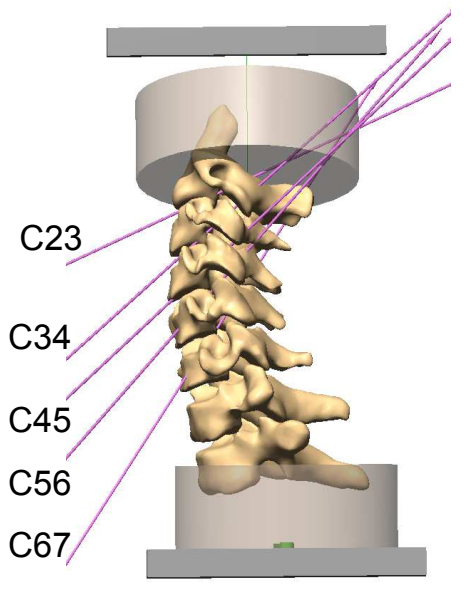

(B)

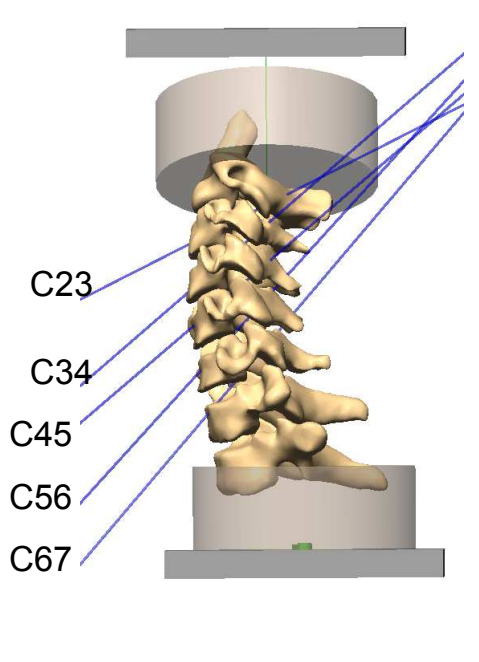

(C)

Figure 3.5 Animation frames of HAM vector analysis. (A) Type 1, (B) Type 2, and (C) Type 3 end constraint conditions. 


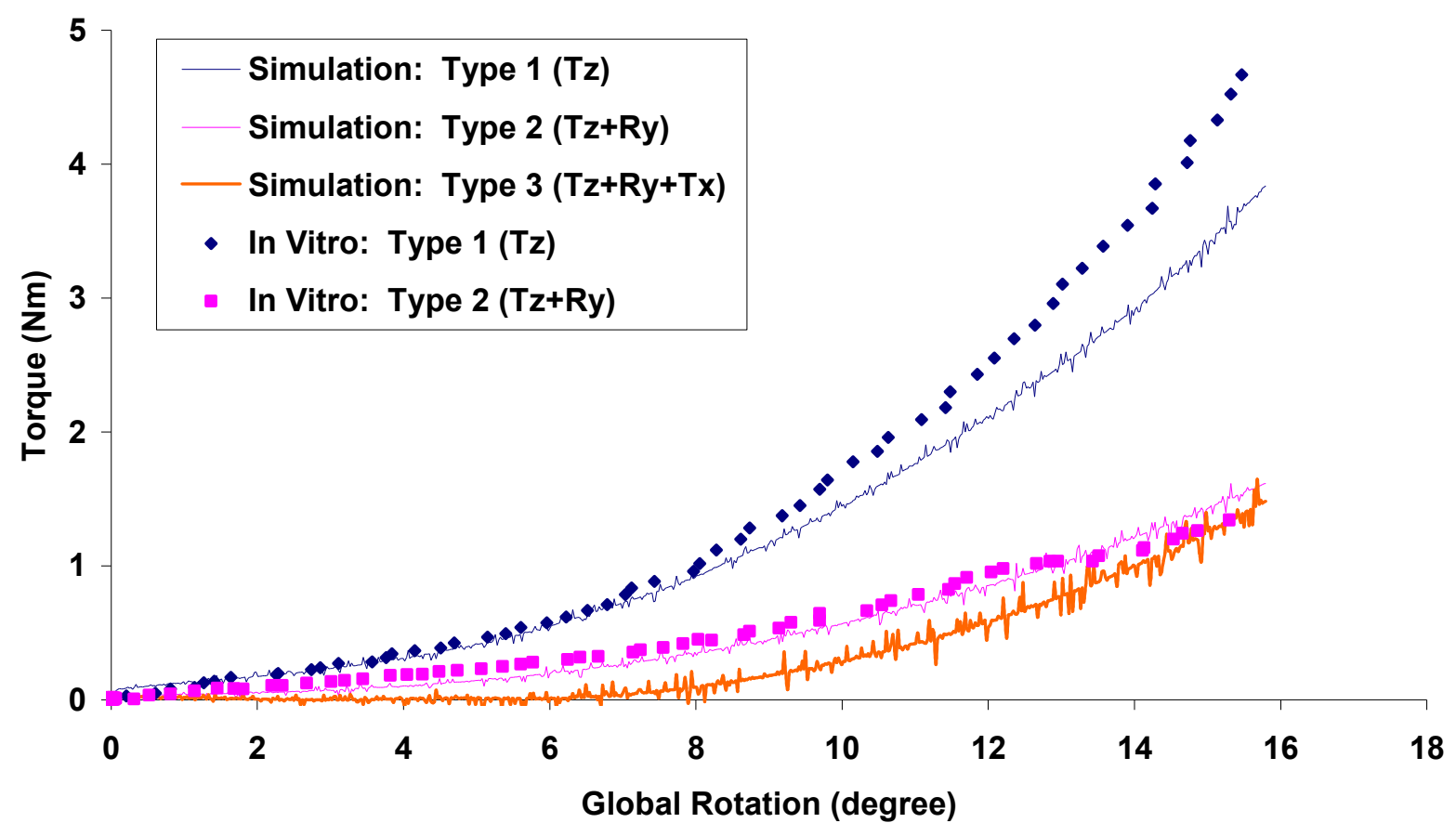

Figure 3.6 Global rotational stiffness curves for the simulation and in vitro tests. 
Type 1 condition and least with the Type 3 . Similar results occurred with the in vitro tests. The reductions of the axial torque between Type 1 and Type 2 conditions at $15^{\circ}$ of global rotation were $4.5 \mathrm{Nm}$ (Type 1) to $1.3 \mathrm{Nm}$ (Type 2) for the in vitro tests and $3.5 \mathrm{Nm}$ to $1.5 \mathrm{Nm}$ for the simulation study.

\subsubsection{Disc and facet forces}

Animations of the force vectors of the discs and facet joints for the three types of end constraint conditions are shown in Figure 3.7 and identified by color-coded force vectors. At the end limit of axial rotation with the Type 1 condition the disc force vectors at the upper motion segment levels (C2-C3, C3-C4, and C4-C5) were angled backward indicative of a larger shear force relative to the compressive force (Figure 3.7). With Type 2 and Type 3 conditions, all the disc force vectors were oriented in a vertical compression direction (Figure 3.7). The facet joint forces were also significantly increased by the end limit of rotation, especially at the upper spinal segments (C2-C3, C3-C4, and C4-C5). The changes in disc forces of Type 2 and Type 3 relative to Type 1 was minimal (Table 3.1). However, the changes in facet forces of Type 2 and Type 3 relative to Type 1 were significant (Table 3.2). 


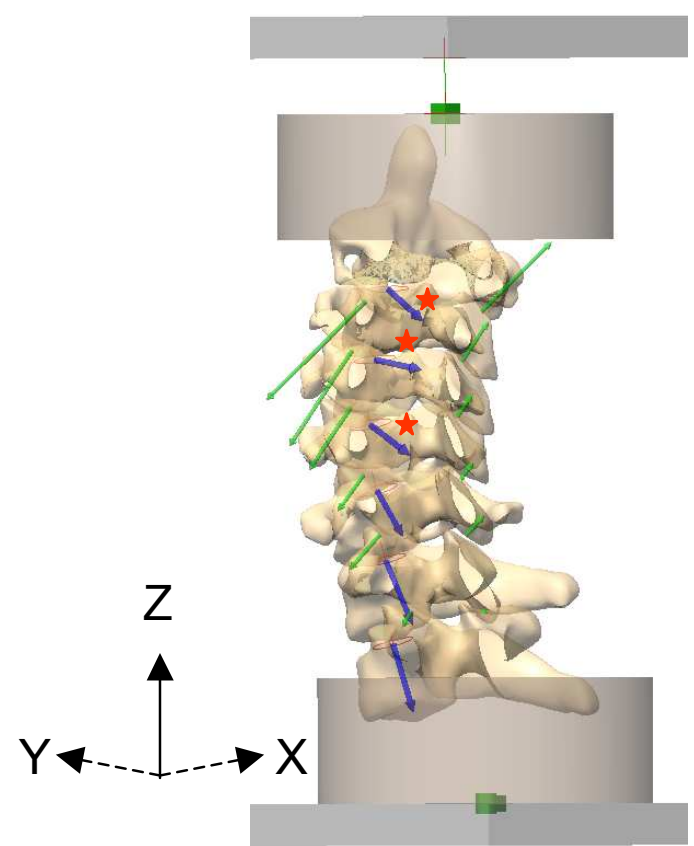

(A)

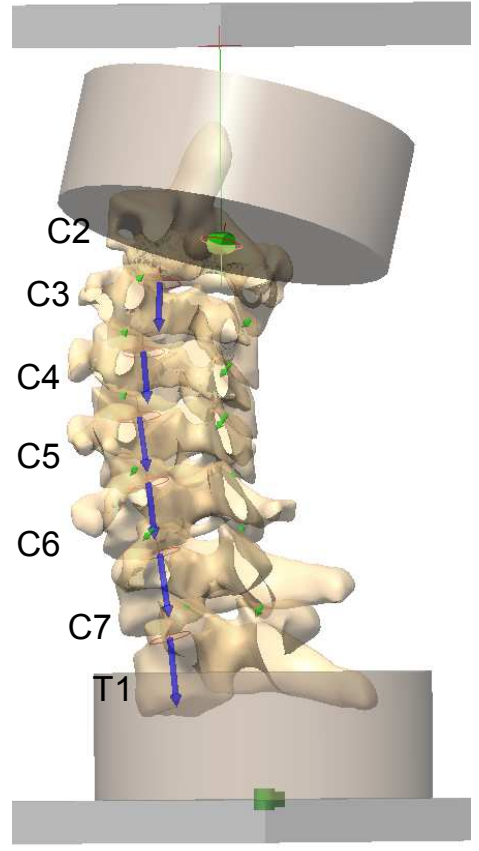

(B)

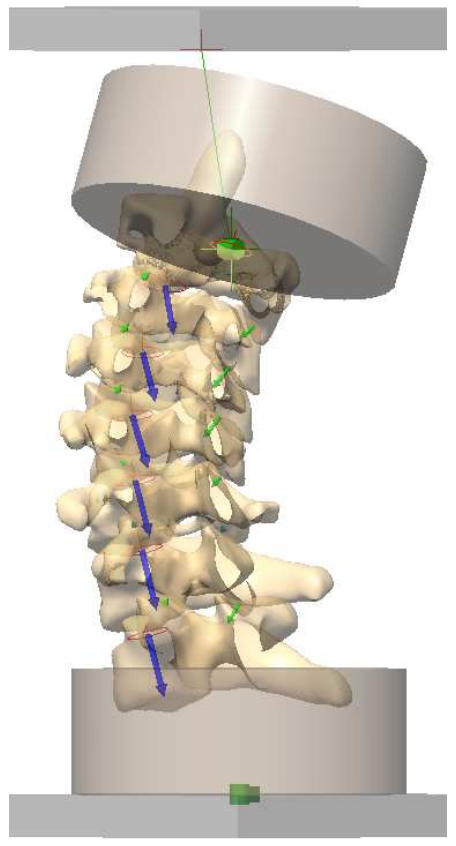

(C)

Figure 3.7 Animation frames of the disc and facet joints force vectors. (A) Type 1, (B) Type 2, and (C) Type 3 end constraint conditions. The arrows illustrate the force vectors: Blue arrow $=$ disc force; Green arrow $=$ facet force of intact spine.

Table 3.1 Disc forces (N).

\begin{tabular}{lccc}
\hline & Type 1 & Type 2 & Type 3 \\
\hline C2-C3 & 24.3 & 24.9 & 24.2 \\
C3-C4 & 25.5 & 26.7 & 25.0 \\
C4-C5 & 25.5 & 28.7 & 26.9 \\
C5-C6 & 25.8 & 29.2 & 28.1 \\
C6-C7 & 33.5 & 32.9 & 29.7 \\
C7-T1 & 34.6 & 34.7 & 31.5 \\
\hline
\end{tabular}


Table 3.2 Facet joint forces $(\mathrm{N})$.

\begin{tabular}{cccc}
\hline & Type 1 & Type 2 & Type 3 \\
\hline C2-C3 (left) & 78.4 & 5.0 & 5.3 \\
C2-C3 (right) & 55.5 & 6.3 & 9.7 \\
C3-C4 (left) & 59.2 & 4.4 & 5.8 \\
C3-C4 (right) & 24.5 & 9.1 & 13.3 \\
C4-C5 (left) & 38.8 & 3.6 & 5.7 \\
C4-C5 (right) & 13.8 & 9.7 & 13.5 \\
C5-C6 (left) & 23.1 & 5.7 & 1.3 \\
C5-C6 (right) & 11.1 & 0.0 & 7.4 \\
C6-C7 (left) & 23.9 & 8.7 & 3.0 \\
C6-C7 (right) & 13.9 & 2.3 & 3.3 \\
C7-T1 (left) & 9.3 & 0.1 & 3.3 \\
C7-T1 (right) & 1.6 & 7.0 & 12.7 \\
\hline
\end{tabular}

\subsection{Discussion}

Traditionally, human cervical spine motion has been described according to the three

anatomical planes: flexion/extension in the sagittal plane, lateral bending in the frontal plane, and axial rotation in the transverse plane (White and Panjabi, 1990). Because of the spatial orientation of the cervical facet joints, unique coupling relationships occur during cervical spine movement (Lysell E, 1969; Milne, 1991; Moroney et al., 1988; Panjabi et al., 1986).

Flexion/extension motion is coupled with anterior/posterior translation and lateral bending is coupled with axial rotation. During flexion and extension, the primary movement is in the 
sagittal plane and the analysis can be reduced to two dimensions. However, lateral bending and axial rotation movements have to be analyzed in three dimensions due to their coupling behavior. The geometry of facet joints is oriented at approximately $45^{\circ}$ to the endplates of the vertebral bodies (Nowitzke et al., 1994; Panjabi et al., 1993) and thus stipulates that the inferior facet must ride up this slope when axial rotation is attempted. As a result, the vertebrae must tilt to the side of the rotation (Bogduk and Mercer, 2000). This knowledge and understanding of cervical kinematics, however, has not been applied well to the in vitro testing protocols that attempt to reproduce physiologic movements.

The Type 1 condition produced non-physiologic kinematic and kinetic responses; opposite directions of coupling, anterior-posteriorly directed HAMs at the C2-C3 segment, and mainly shear forces at the disc joints of upper segments (C2-C3, C3-C4, and C4-C5). By allowing more degrees of freedom at the end mounts, the simulation model response more closely followed the physiologic pattern. The coupling ratios of the Type 2 condition were closer to those of Panjabi et al. (2001) than the Type 3 condition. This finding is a result of the Type 2 condition being similar to the testing method used by Panjabi et al. (2001). The HAMs vectors of the Type 2 mounting configuration were located in the upper moving vertebral bodies, indicating that there was rocking motion in the concavity of superior surface of vertebral bodies. The HAMs vectors of the Type 3 mounting configuration were located in the lower vertebral bodies, indicating that 
there was rotational and translational movement. Both Type 2 and Type 3 conditions simulate some part of physiologic axial rotation motion, but they do so in different ways.

Although the mounting arrangements and end conditions between the simulation and the in vitro experimental set-up are different (i.e., upright spine in the simulation model versus an inverted alignment in the in vitro set-up), the axis of rotation through $\mathrm{T} 1$, the vertical translation of $\mathrm{C} 2$ relative to $\mathrm{T} 1$, and the lateral rotation of $\mathrm{C} 2$ are fundamentally identical.

Analysis of the in vitro data from the Type 1 condition revealed un-physiologic coupling patterns as observed in the simulation. The $\mathrm{C} 2$ vertebral body rotated laterally to the opposite side of axial rotation. With the addition of lateral rotation at C2 (Type 2), all vertebral bodies of the in vitro specimen rotated in a direction consistent to the direction of axial rotation. The addition of a linear slider to provide the Type 3 condition produced results identical to that of the Type 2 condition. The amount of lateral force produced from the coupling effect was not large enough to overcome the frictional forces in the linear bearing assembly. Based on the coupling patterns and physiologic motion responses with the Type 2 condition compared to the Type 3 arrangement, the translational degree-of-freedom (Type 3) was not necessary.

In summary, use of dynamic computer simulation techniques effectively demonstrated the current limitations with existing in vitro axial rotation testing protocols. The virtual model can now be readily used to explore and modify different in vitro testing methods. Animation of disc 
and facet joint forces also provided further insight into the kinetic effects of the end constraint conditions during axial rotation testing. 


\section{Chapter 4}

\section{Biomechanics of Single-level Cervical Fusion Using a Virtual Dynamic Spine Model}

\subsection{Introduction}

Cervical spine fusion is an established surgical procedure to eliminate neck pain and to provide stability to patients suffering degenerative disease and traumatic injury (Connolly et al., 1965; Gore and Sepic, 1984; Green, 1977; Herkowitz et al., 1990; Lindberg, 1970). In spite of the high fusion rate and good to excellent clinical outcome of cervical spine fusion (Brodke and Zdeblick, 1992; Emery et al., 1994; Gore and Sepic, 1984), it has been shown that the adjacent level to the fusion has an increased incidence of symptoms (Hilibrand et al., 1999; Hilibrand et al., 1997) and degeneration (Baba et al., 1993; Braunstein et al., 1980; Hunter et al., 1980). Although the complete mechanisms behind the adjacent segment degeneration have not been revealed yet, several biomechanical studies have reported increased movement (Baba et al., 1993; DiAngelo et al., 2000), intradiscal pressure (Eck et al., 2002; Pospiech et al., 1999), and strain (Matsunaga et al., 1999) at segments adjacent to the fusion level. Although these 
mechanical changes may not be the entire reason for the adjacent segment problems after cervical spine fusion, the influence of these mechanical changes cannot be ignored.

The cervical spine can be modeled as a mechanical structure and analyzed to give a more theoretical explanation of the mechanics of the cervical fusion. While there are many computer models developed to study biomechanics of cervical spine, there are virtually no papers using computer models for investigating the controversial mechanism of degeneration of adjacent segments to the fusion level. Currently the only available study using a computer model to study biomechanical effects of fusion is a two motion segment (C4-C5-C6) finite element (FE) model (Maiman et al., 1999). Although the FE analysis demonstrated the possibility of a computer model to delineate the internal responses of the adjacent segment to a fusion level, the FE model study was limited to only two motion segments and the loading condition was not physiologic. Furthermore, the FE method is suited for performing a static structural analysis, but is not ideal for investigating large-scale dynamic movements of multi-body structures. Forward dynamic multi-body simulations have been successful at examining joint loads during large movements; applications include knee and hip joints (Gerritsen et al., 1996; Piazza and Delp, 2001; Arnold et al., 2000; Neptune and Kautz, 2000; Neptune et al., 2000). To date, however, no forward dynamic multi-body simulation study has been performed that studies the kinetics and kinematics of the cervical spine following single-level cervical fusion. This study included two 
parts: an in vitro study (Schwab, 2003) to measure the relative changes in the contribution of each motion segment unit and the global moment-rotation behavior after single-level a C5-C6

fusion. A forward dynamic simulation model of the same in vitro testing protocol was developed to study the biomechanical changes brought on by single-level fusion at $\mathrm{C} 5-\mathrm{C} 6$. The simulation results were compared with those from in vitro testing for model evaluation. Subsequently, the vector animation and analysis of the disc and facet forces were undertaken.

This study should provide the clinician and spine researcher with a theoretical paradigm for understanding cervical fusion mechanics in a more intuitive and visual manner.

\subsection{Methods}

\subsubsection{In vitro testing methods}

Data were collected for flexion, extension, lateral bending, and axial rotation testing of the sub-axial human cervical spine in the intact and fused conditions. Fusion was simulated at the C5-C6 level. The in vitro testing protocol applies a moment distribution across the cervical spine that induces a more physiologic motion response across the spine (DiAngelo and Foley, 2003). Six fresh human cadaveric cervical (C2-T1) spines were procured, prepared and screened for abnormalities. The specimens were mounted in a programmable testing apparatus and tested in 
flexion, extension, lateral bending and axial rotation. The testing system had an upper fixture, consisting of a linear bearing and splined shaft assembly, that mounted in a rotational joint attached to a vertical actuator, as shown in Figure 4.1. The specimen was placed in an inverted neutral orientation eccentric to the load axis of the actuator. The actuator applied a compressive load and bending moment to the upper mounting pot. For axial rotation, the lower mounting pot was free to rotate axially and unconstrained in lateral tilt (Ry) and lateral translation (Tx), while the upper mounting pot was unconstrained in the vertical direction $(\mathrm{Tz})$. The spines were loaded under displacement control.

\subsubsection{Construction of the model and simulation task}

An existing three-dimensional computer graphics-based model of sub-axial cervical spine was used. The simulation model was previously described in detail.

The computer model simulated flexion, extension, lateral bending, and axial rotation motion of sub-axial human cervical spine of the intact and fused spine conditions. The boundary conditions were modeled from an in vitro testing protocol (DiAngelo and Foley, 2003; Foley et al., 1999). The virtual experimental set-ups are illustrated in Figure 4.1. For flexion, extension, and lateral bending, the simulated fixtures were created to mimic the in vitro fixtures as described above. This induced a compressive load and bending moment to the upper mounting 
(A)
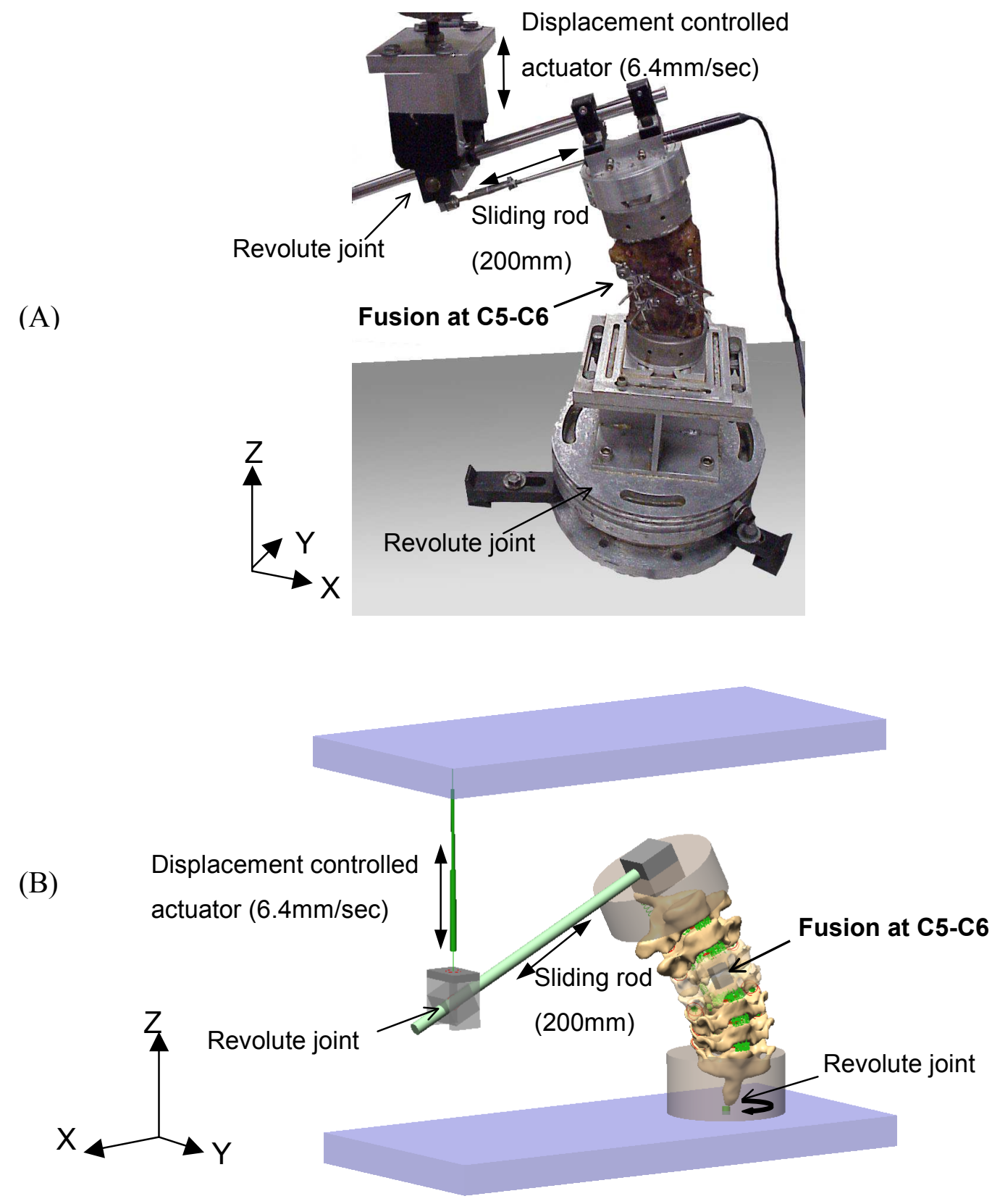

Figure 4.1 The in vitro experimental testing apparatus and animation frames of the virtual experimentation of a single-level fusion at the C5-C6 level. (A) In vitro testing set-up and (B) Virtual testing set-up. 
pot. For axial rotation, the cervical spine was placed in an upright position. A revolute motor located at the center of the lower mounting pot provided axial rotation while the upper mounting pot was unconstrained in the vertical direction (Tz), lateral tilt (Ry), and lateral translation (Tx). This produced an analogous end conditions as found in the in vitro testing protocol.

Displacement control methods were used to apply a moment distribution across the cervical spine model. The end limits of rotation for flexion, extension, lateral bending, and axial rotation were $30^{\circ}, 24^{\circ}, 26^{\circ}$, and $15^{\circ}$, respectively. Fusion at the $\mathrm{C} 5$-C 6 level was modeled as a rigid joint, with no motion allowed between C5 and C6.

\subsection{Results}

\subsubsection{Changes in motion segment unit rotations}

The percent change in the contribution of each motion segment unit before and after the C5C6 fusion for the in vitro tests and simulation study are shown in Figure 4.2. The simulation results were similar to results from the in vitro testing and showed that the virtual model responded similarly to the in vitro testing. Following fusion, all segments provided some level of compensation. During simulated flexion, more compensation occurred in the upper adjacent segment (C4-C5) and during extension more compensation occurred at the lower adjacent 
(A)

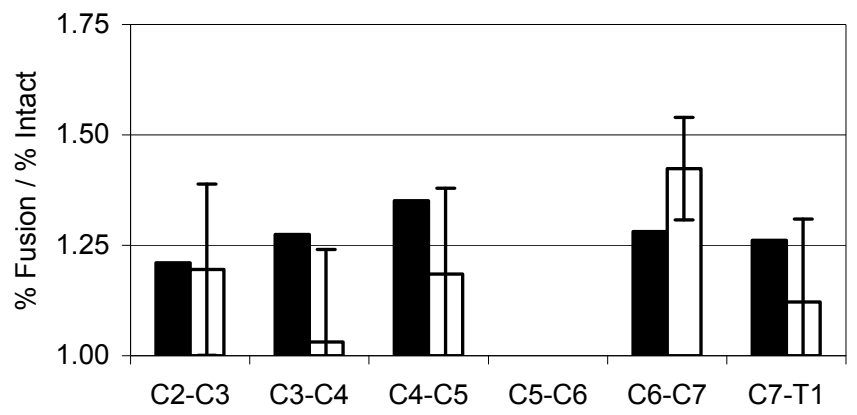

(B)
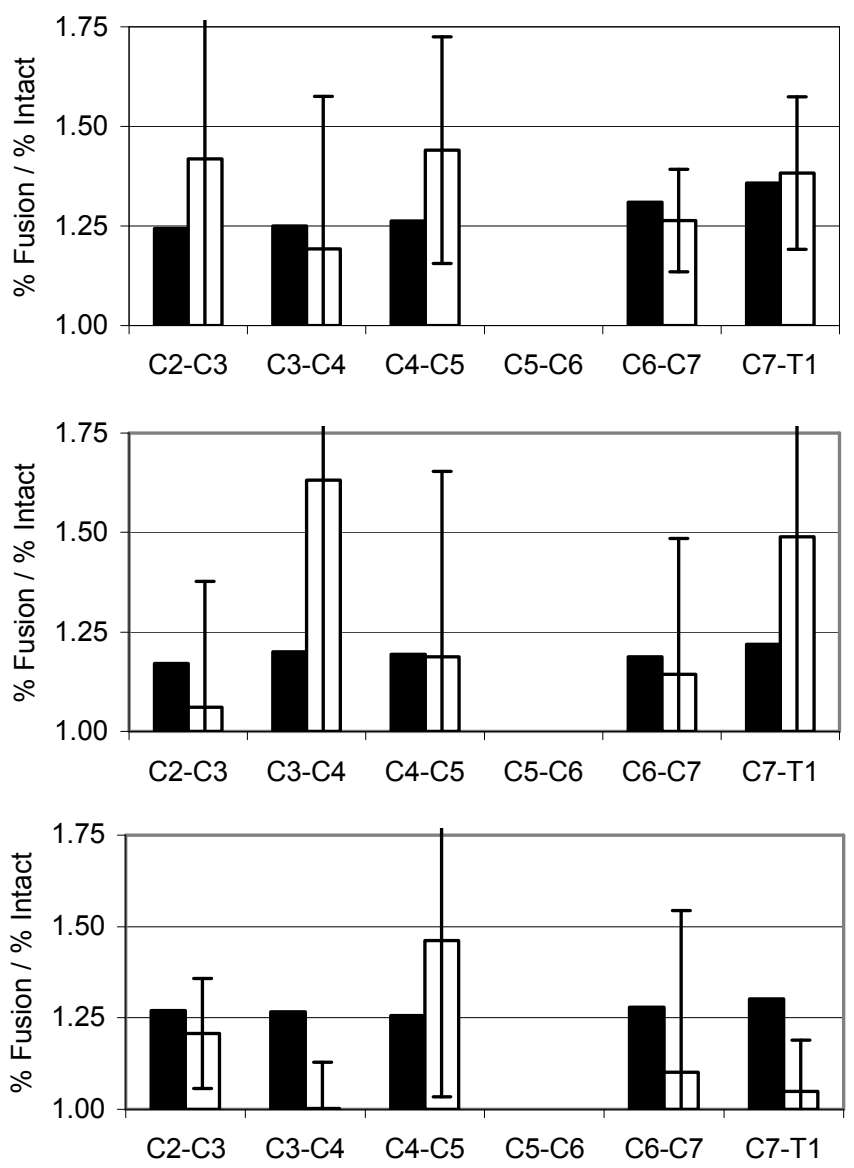

Simulation

$\square$ In Vitro

Figure 4.2 The change in percent contribution of rotation before and after $\mathrm{C} 5-\mathrm{C} 6$ fusion between the in vitro testing and simulation studies. (A) Flexion, (B) Extension, (C) Lateral bending, and (D) Axial rotation. All intact segments shared in the motion compensation in different amounts. During flexion, more compensation occurred in the upper adjacent segment (C4-C5) and more compensation occurred in the lower segments (C6-C7 and C7-T1) during extension. During lateral bending and axial rotation, a more uniform response occurred with no specific motion compensation pattern. 
segments (C6-C7 and C7-T1). During simulated lateral bending and axial rotation, however, there was no specific pattern for the motion compensation.

\subsubsection{Global stiffness patterns}

Typical in vitro global rotational stiffness curves before and after C5-C6 fusion are shown in

Figure 4.3. Fusion caused a notable increase in rotational stiffness. The stiffness response increased in a non-linear hyperbolic manner and the length of the low stiffness regions was shortened.

In the simulation study, the end moment limit increased 2 to 4 times that of the intact condition (3.98 times for flexion, 3.85 times for extension, 2.32 times for lateral bending, and 2.10 times for axial rotation) for all loading modes. In the in vitro experimental data, the global stiffness values also increased in a non-linear hyperbolic manner and the regions of low stiffness were decreased (Figure 4.4). The percent reduction of the low stiffness regions were $30 \%, 30 \%$, $20 \%$, and $40 \%$, for flexion, extension, lateral bending, and axial rotation loading respectively.

\subsubsection{Segmental force vector analysis}

The differences in the magnitude of the disc forces at each vertebral level before and after fusion are shown in Figure 4.5. Notable increases in the disc forces were observed during 

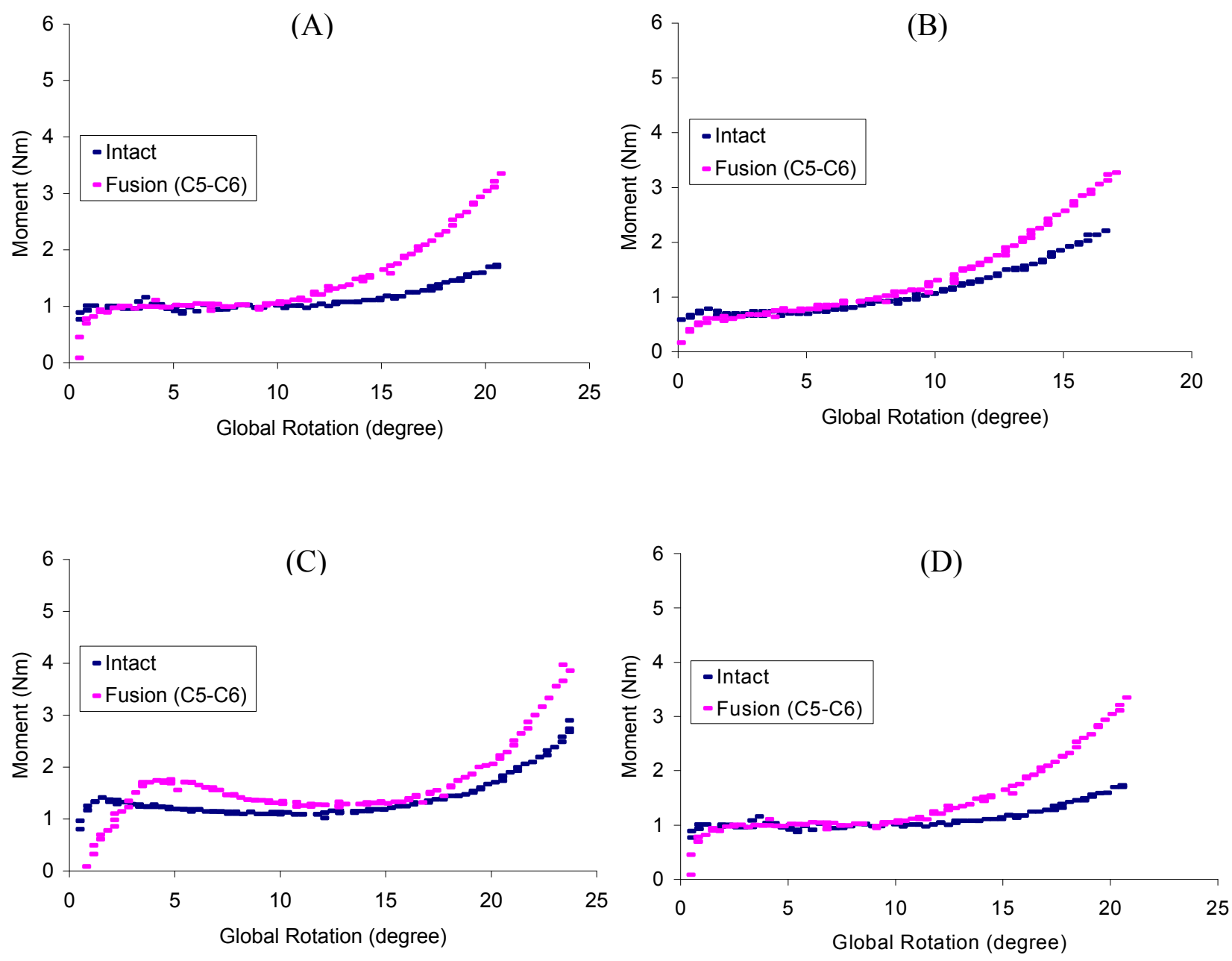

Figure 4.3 Typical in vitro global rotational stiffness curves before and after fusion (C5C6). (A) Flexion, (B) Extension, (C) Lateral bending, and (D) Axial rotation. 

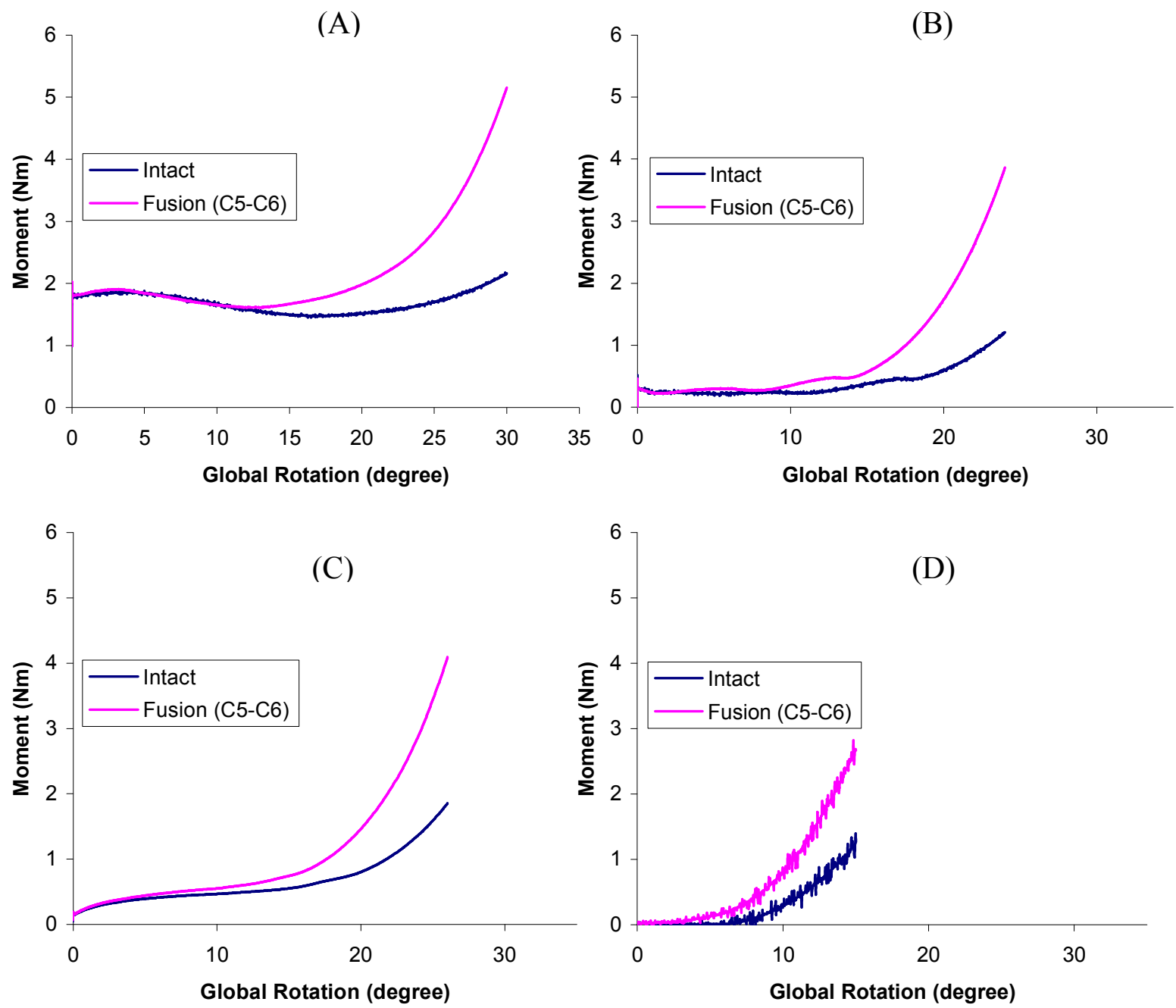

Figure 4.4 The simulation results of global rotational stiffness curves before and after fusion (C5-C6). (A) Flexion, (B) Extension, (C) Lateral bending, and (D) Axial rotation. The global stiffness of the fused spine increased parabolically once a certain amount of rotation was reached. As a result, use of global stiffness data may not show differences between pre- and post-surgical conditions if the applied loads are not sufficient. 
(A)

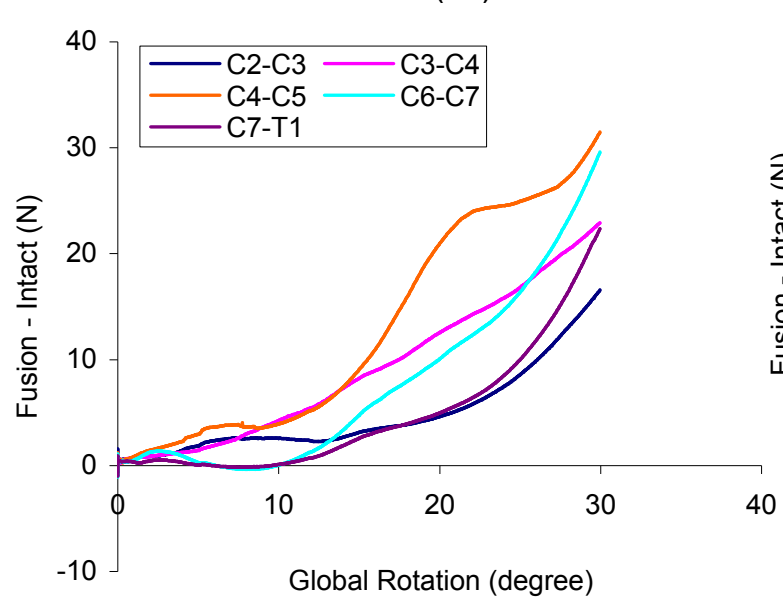

(C)

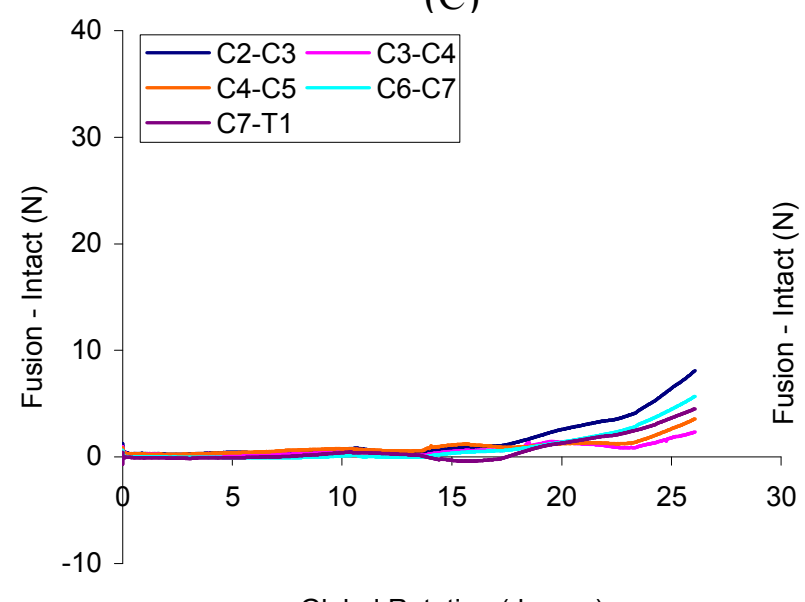

Global Rotation (degree)

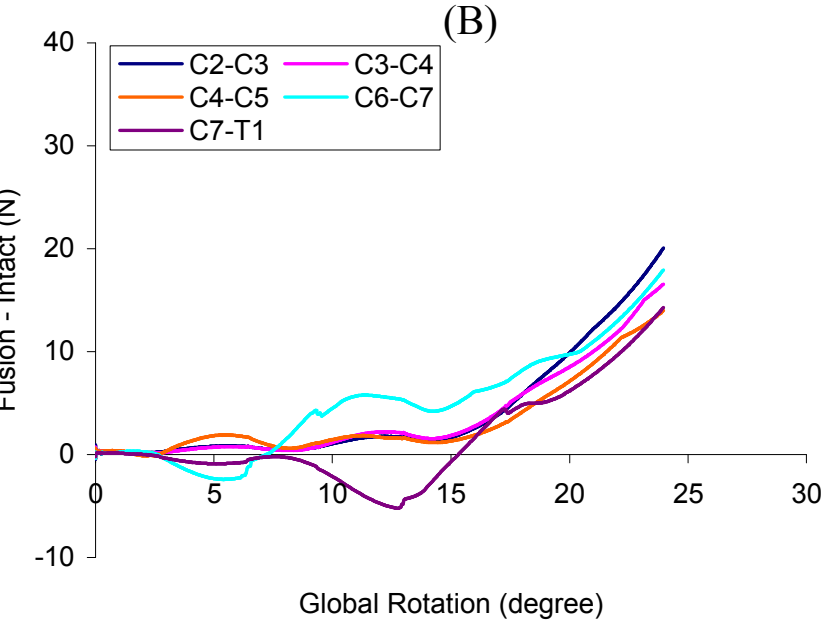

(D)

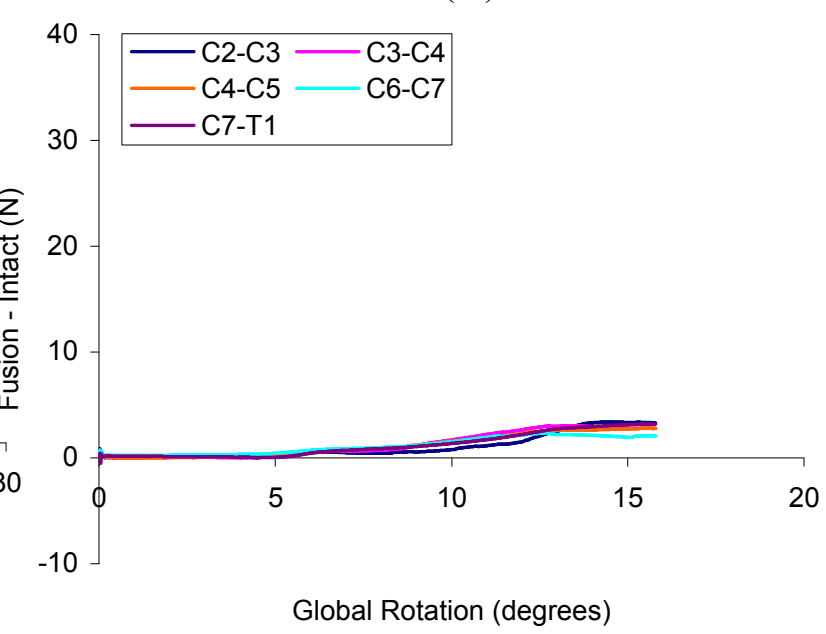

Figure 4.5 The differences in magnitude of the disc translational force before and after fusion (C5-C6). (A) Flexion, (B) Extension, (C) Lateral bending, and (D) Axial rotation. 
flexion and extension. Further, during flexion loading, fusion increased the disc force the most at $\mathrm{C} 4-\mathrm{C} 5$ and $\mathrm{C} 6-\mathrm{C} 7$ and least at the $\mathrm{C} 2-\mathrm{C} 3$ level. During extension loading, the fused condition caused the greatest increase in disc force at $\mathrm{C} 2-\mathrm{C} 3$ level, which gradually decreased across the remaining levels. However, the differences between segments were not significant. During lateral bending, minimal changes occurred in the disc forces following fusion until some amount of rotation occurred (i.e., $20^{\circ}-23^{\circ}$ ). During axial rotation loading, fusion had no effect on the disc force.

The segmental disc and facet joint force vectors for the animation frames at the end limits of flexion, extension, lateral bending, and axial rotation are shown in Figure 4.6. Color-coding was applied to each of the vectors for comparison of the forces before and after fusion. Blue, red, green, and pink arrows represent the disc force vectors before fusion, disc force vectors after fusion, facet force vectors before fusion, and facet force vectors after fusion, respectively. During flexion loading, the disc force vectors after fusion were collinear with disc force vectors before fusion, with the disc force vector having greater length. Facet force vectors were small in length, which meant there were negligible forces across the facet joints even after fusion. During extension loading, the disc forces after fusion were also collinear with disc forces before fusion, but with greater magnitudes (i.e., longer vectors). Facet force vectors also increased in length, but the changes were not significant. During lateral bending loading, the shear component of the 


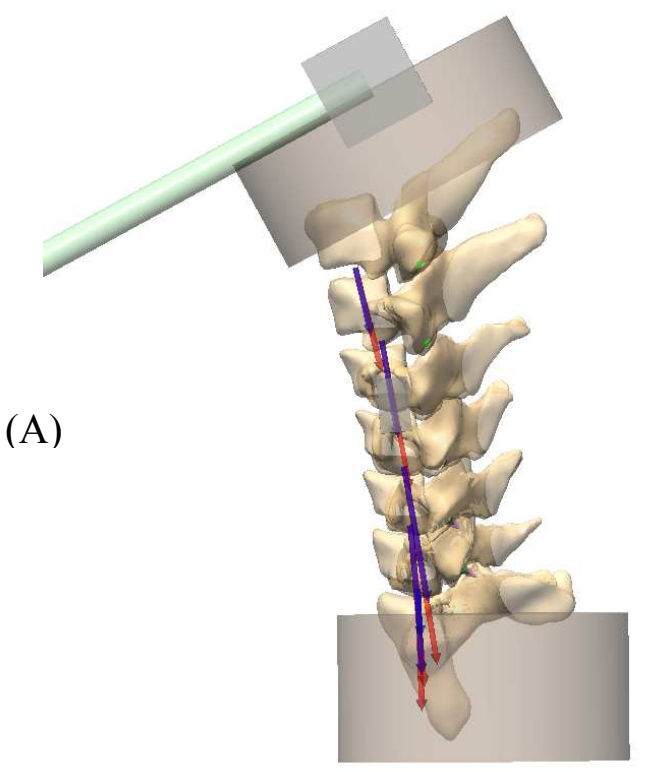

(C)

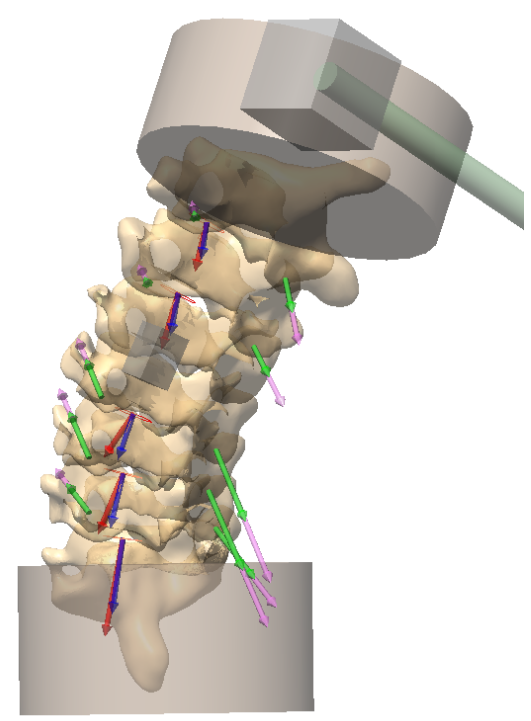

(B)

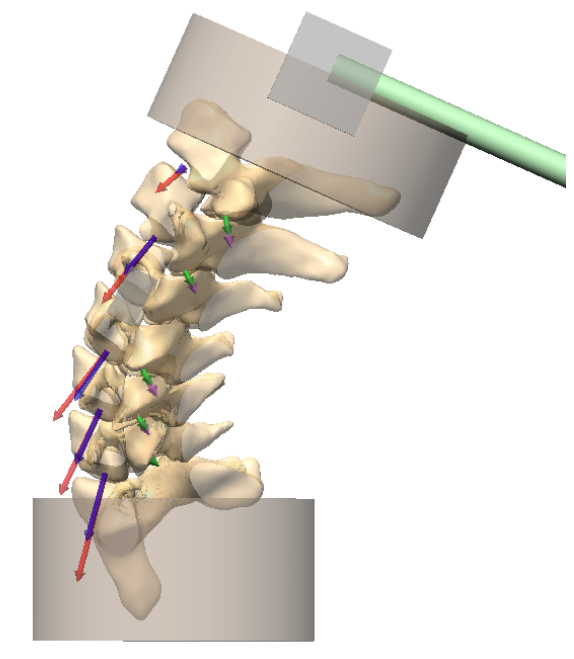

(D)

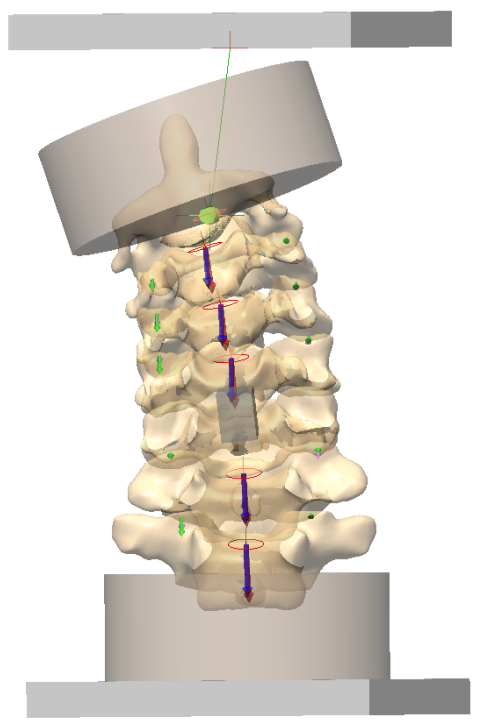

Figure 4.6 Animation frames of segmental disc and facet translational forces. (A) Flexion, (B) Extension, (C) Lateral bending, and (D) Axial rotation. The arrows illustrate the force vectors: Blue arrow $=$ disc force before fusion; Red arrow $=$ disc force after fusion; Green arrow = facet force before fusion; Pink arrow $=$ facet force after fusion. 
disc forces increased, especially at the $\mathrm{C} 3-\mathrm{C} 4$ and $\mathrm{C} 4-\mathrm{C} 5$ levels, representative of an anteriorly tilted force vector. Facet forces during lateral bending loading were greatest among all loading conditions. After fusion the facet joint forces increased by $50 \%$ to $90 \%$ during lateral bending. The direction of facet force vectors indicated that the left-sided facets were under compression and right-sided facets were under tension. During axial rotation there was no significant change in the force vectors of the disc and facet joints before and after fusion.

\subsection{Discussion}

A computer simulation study was presented that analyzed the kinematic and kinetic response following single-level cervical fusion. To the authors' knowledge, the only other computer model of cervical spine fusion was a two motion segment unit FE model developed by Maiman et al. (1999). Unfortunately, with only two MSUs in the model, it was not possible to determine the level of compensation between the upper and lower adjacent segments. In addition, simulation of physiologic loads was not possible. A multi-segment sub-axial cervical spine model was developed in a graphics-based modeling platform that included the remaining upper (C2-C3, C3-C4, and C4-C5) and lower cervical segments (C6-C7, C7-T1). As such, any compensation or involvement of those segments to a single-level fusion could be included. 
Furthermore, by simulating an experimental testing protocol, which has been shown to induce a more physiological motion response across the cervical spine during in vitro testing (DiAngelo and Foley, 2003), a better understanding of the motion compensation resulting from fusion occurred. This should also provide a more accurate approach to modeling the connective tissue structures of the cervical spine, as reflected in the global stiffness curves and the percent contributions of rotation.

\subsubsection{Motion compensation}

In a study examining C5-C6 fusion, Eck et al. (2002) reported that the adjacent segment motion increased at both ends during flexion; C4-C5 motion increased more (by 32.5\%) than C6C7 (by 22.3\%), but did not reach a statistical significance. They also reported that during extension, the change was significantly different at C6-C7 (19\% increase). This pattern of motion compensation was consistent with our simulation results (Figure 4). During lateral and axial rotation testing, all remaining motion segments provided some compensation for the decreased motion at the fusion site. 


\subsubsection{Global stiffness response}

Fuller et al. (1998) reported a non-linear increase in the bending stiffness properties during sagittal plane rotation for different levels of fusion configurations (i.e., zero to three motion segment units). In our simulation study, the moment required to achieve similar endpoint rotations also increased in a non-linear fashion, characteristic of the biological tissue. Also, the global neutral zone of the sub-axial cervical spine markedly decreased following a single-level fusion.

\subsubsection{Segmental disc and facet joint force vectors}

The increase in adjacent segment disc forces after C5-C6 fusion coincided with the intradiscal pressure measurements reported by Eck et al. (2002) during flexion/extension. The same end limits of rotation used by Eck et al. (2002) ( $20^{\circ}$ flexion and $15^{\circ}$ extension) were used in the simulation study and resulted in greater disc forces at the level superior to the fusion (C4-C5) during flexion loading and inferior to the fusion (C6-C7) during extension. Single-level fusion did not affect the disc forces during lateral bending and axial rotation motion.

The novelty of our model came from the animation of force vectors at the disc and facet joints during the motion simulation. Matsunaga et al. (1999) reported that no statistical increase of shear strain in intervertebral discs was observed during flexion and extension motion after 
surgery in cases of single-level fusion. This simulation study also showed similar findings

during flexion and extension motion. The disc force vectors after the C5-C6 fusion were well

aligned with the force vectors before fusion. However, during lateral bending motion, which

was not studied by Matsunaga et al. (1999), increases in the anterior shear components of disc

forces occurred at the superior levels (C4-C5 and C3-C4) only. These shear forces may enhance

the degenerative process at the adjacent segments.

\subsubsection{Limitations}

One limitation of this study, inherent in all computer modeling of biologic systems, is the difficulty to accurately model the non-linear properties of the tissue and surrounding ligamentous structure.

A rigid, multi-body, large displacement theory dynamical approach was used to investigate cervical fusion mechanics. Although this approach has definite advantages over FE model, the simulation model cannot predict the changes of internal disc and vertebral stress distributions after fusion. Changes in the forces were assumed to correlate with the stresses in the tissue and correspondingly effect tissue degeneration after fusion. Further, during the process of simulating fusion, the motion segment unit was idealistically modeled as an immovable rigid joint, representative of a fully ankylosed joint. However, in the biomechanical testing realm, 
instrumentation is often used to simulate fusion and does not completely immobilize the fused region. Small amounts of motion may occur affecting the global stiffness and kinematic responses of the fused spine in vitro. However, in the simulation model, fusion was more representative of the in vivo situation. Physiologically, ligaments of the spine span multiple segments, and when fusion occurs and motion ceases across a motion segment unit, the ligaments attached to the other mobile bodies are affected by the fusion. In the simulation model, the ligaments originated at one vertebral body and terminated at the adjacent vertebral body, spanning a single motion segment unit. Simulation of ligaments spanning multiple segments was not possible.

\subsubsection{Conclusions}

A multi-body dynamic simulation model of the sub-axial cervical spine was used to study the biomechanical effects of single-level cervical spine fusion. Fusion increased the motion compensation at the adjacent segments during flexion and extension motion and increased the global rotational stiffness for all four modes of loading. The disc forces increased at the level superior to the fusion (C4-C5) during flexion and inferior to the fusion (C6-C7) during extension. Lastly, animation of the force vectors provided an alternative method for demonstrating the biomechanical response to single-level fusion. 


\section{Chapter 5}

\section{Dynamic Simulation of the Effect of Different Disc Arthroplasty Designs on Cervical Spine Biomechanics}

\subsection{Introduction}

Cervical disc arthroplasty has recently gained acceptance as an alternative to fusion surgery

for preserving and/or restoring motion at the treated level. Although initial clinic experiences have reported some degree of motion restoration (Cummins et al., 1998; Goffin et al., 2002;

2003; Sekhon, 2003; Wigfield et al., 2002a; 2002b), the overall success rates have not been as favorable as hip or knee arthroplasty (Sengupta, 2004). The limited success of spine arthroplasty relative to hip/knee arthroplasty may, in part, be due to the added complexity of the multi-body structure of the spine. Each spinal motion segment unit (MSU) consists of three main joints: an intervertebral disc and two facet joints, and center of rotation of each MSU constantly moves along the three axes (Gunzburg et al., 2002). Thus, designing an artificial disc implant that reproduces this complex structure and restores natural motion without causing joint impingement or dislocation becomes a formidable design challenge. 
Many different cervical disc designs have been proposed and can be classified as those that reproduce the viscoelastic properties of the disc or the motion characteristics of the disc (Szpalski et al., 2002). A common disc arthroplasty design for restoring motion is a constrained or semi-constrained "ball-and-socket" type device (Lee and Goel, 2004; Niosi and Oxland, 2004). Some examples of this type of cervical disc design (Le et al., 2004) include the Prestige cervical disc (Medtronic Sofamor Danek, Inc., Memphis, TN), the CerviCore (SpineCore, Summit, NJ), the Porous Coated Motion (PCM) (CerviTech, Roundhill, NJ), and the ProDisc-C (Synthes Spine Solution, Paoli, PA). The Prestige disc has an ellipsoid saucer on the lower component that articulates with a domed superior component (Singh et al., 2004; Smith et al., 2004; Traynelis, 2004). The CerviCore disc has opposing bearing surfaces that have fixed centers of rotation below the bottom base plate during flexion/extension and above the top base plate during lateral bending (Smith et al., 2004). The PCM disc consists of two endplates with a bearing surface attached to a caudal endplate that has a larger radius of articulation and permits translation through a rotational arc (Smith et al., 2004). The ProDisc-C is similar to a constrained ball-and-socket joint and provides unconstrained rotation but no translation (DiAngelo et al., 2004; Link et al., 2004; Singh et al., 2004).

A limited number of in vitro studies of spinal disc arthroplasty devices for the lumbar spine (Buttner-Janz et al., 1989; Cunningham et al., 2002; Lee and Goel, 2004) and the cervical spine 
(DiAngelo et al., 2003; 2004) have been reported to date. However, no theoretical models exist that evaluate the design paradigms for disc prostheses (Anderson and Rouleau, 2004). Clinical experiences of large joint arthroplasty have demonstrated that proper placement of a device with fewer motion constraints provides for better restoration of natural kinematics and kinetics (Anderson and Rouleau, 2004). To date, computational models of the cervical spine have traditionally employed finite element methods to study the static structural behavior of the intact spine before and after application of fusion instrumentation or to characterize a particular spinal disease or instability. Few computational studies have investigated the mechanics of cervical disc arthroplasty. Dooris et al. (2001) performed a finite element analysis (FEA) of the osteoligamentous L3-L4 motion segment and found that changes in facet loads occurred by altering placement of the artificial disc in the anteroposterior direction. To computationally study the kinematics and kinetics of disc arthroplasty devices, a multi-body dynamic simulation model should be employed, similar to that performed in knee and hip joint studies (Arnold et al., 2000; Gerritsen et al., 1996; Neptune and Kautz, 2000; Neptune et al., 2000; Piazza and Delp, 2001). No computational models exist that study the kinematics and kinetics of cervical disc devices. 
The purpose of this study was to determine the biomechanical effects of different design features for cervical disc arthroplasty using a physics-based dynamic simulation model of the sub-axial cervical spine.

\subsection{Methods}

\subsubsection{Simulation of in vitro testing protocol}

A previously developed three-dimensional graphics-based computer model of the sub-axial cervical spine was used to study the mechanics of a C5-C6 disc implant.

An in vitro testing protocol was developed that replicated the in vivo motion response of the sub-axial cervical spine under flexion, extension, lateral bending, and axial rotational loading (DiAngelo and Foley, 2003; 2004; Foley et al., 1999). The computational model was designed to first simulate our in vitro testing protocol. The upper fixture consisted of a linear bearing and splined shaft assembly mounted in a rotating joint attached to a vertical actuator. For flexion, extension, and lateral bending, the cervical spine was placed in an inverted neutral orientation eccentric to the load axis of the actuator. This induced a compressive load and bending moment to the upper mounting pot. For axial rotation, the cervical spine was placed in an upright position. A revolute motor located at the center of the lower mounting pot provided axial 
rotation while the upper mounting pot was unconstrained in the vertical direction (Tz), lateral tilt (Ry), and lateral translation (Tx). Displacement control methods were used to apply a moment distribution across the cervical spine model. The end limits of rotation for flexion, extension, lateral bending, and axial rotation were $37^{\circ}, 28^{\circ}, 30^{\circ}$, and $16^{\circ}$, respectively.

Three different prosthetic disc design (PDD) variations were studied with the virtual model and compared to the intact spine. They were 1) PDD-I: a 3 degree-of-freedom (DOF) spherical joint located at the mid C5-C6 disc, 2) PDD-II: a 3-DOF spherical joint located $6.5 \mathrm{~mm}$ below the mid C5-C6 disc, and 3) PDD-III: a 5-DOF spherical joint in plane located at C5-C6 disc level (Figure 5.1).

The surgical procedure for implantation of the disc prosthesis consisted of resecting the anterior longitudinal ligament (ALL), the anterior part of the annulus and the nucleus pulposus. This was simulated in the computer model by removing the three spring-damper element of the anterior longitudinal ligament and tissue structure at the C5-C6 MSU. To accommodate the changes in the rotational stiffness properties that result from the implant surgery, stiffness values (or inverse of flexibility) from our in vitro studies of the ProDisc-C implant were used. The stiffness values of the implanted condition relative to the harvested condition were $90 \%$ in flexion, $50 \%$ in extension, $90 \%$ in lateral bending, and $50 \%$ in axial rotation. 
(A)

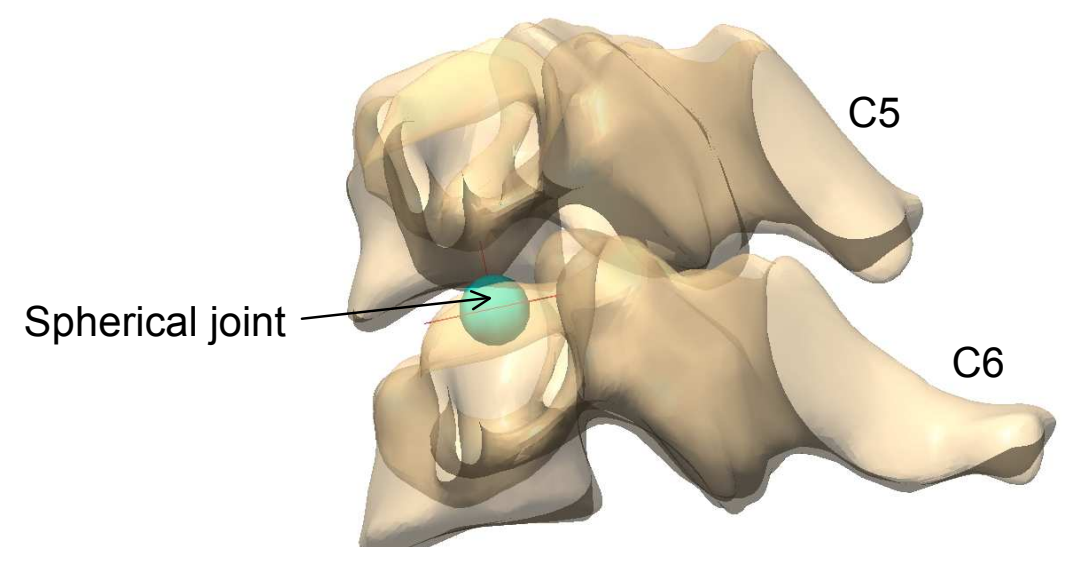

(B)

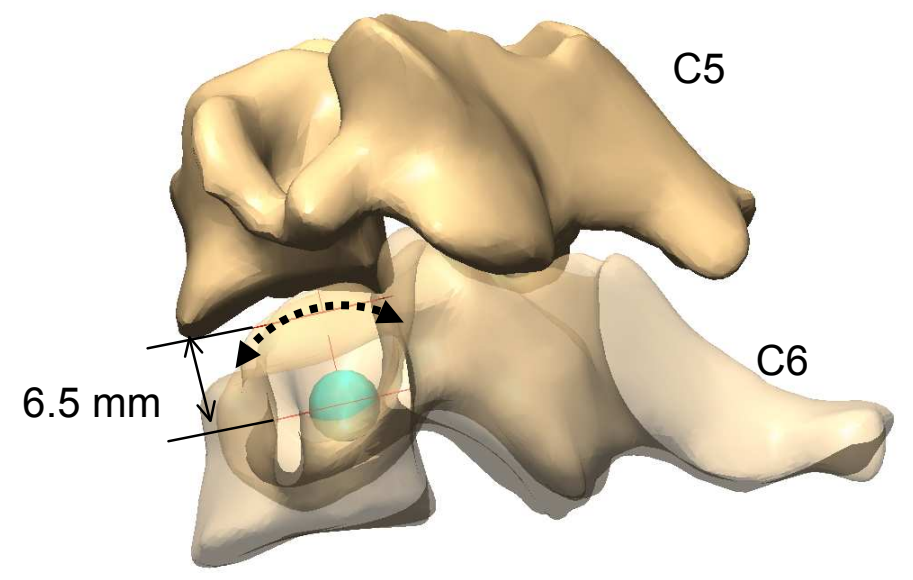

(C)

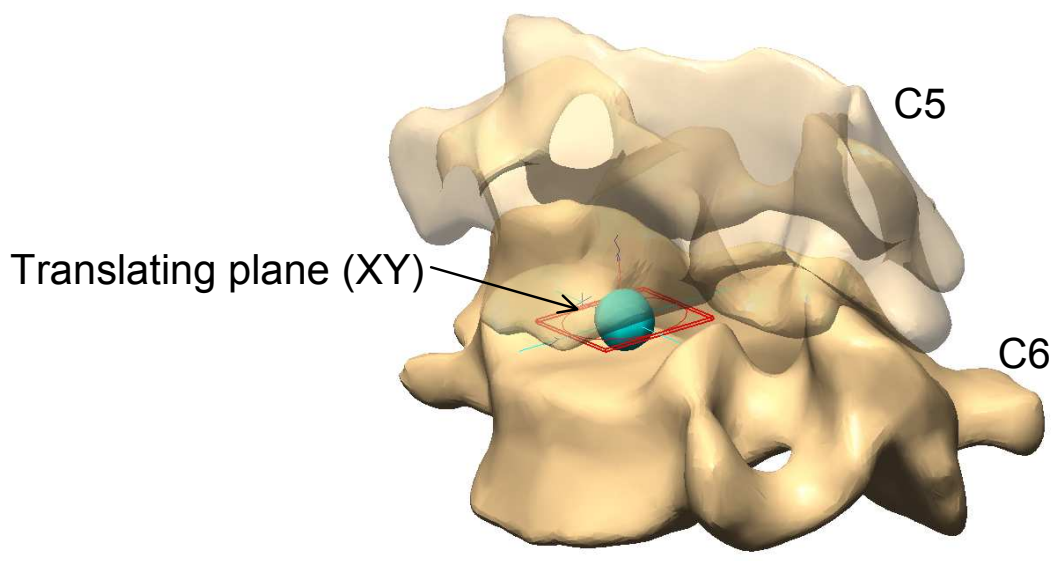

Figure 5.1 Three different design variations of the prosthetic disc. (A) PPD-I (Prosthetic Disc Design-I), (B) PDD-II, and (C) PDD-III. 


\subsubsection{Evaluation of the virtual spine model}

The kinematic analysis included a comparison of the percent contribution of rotation of one MSU relative to the total (C2-T1) rotation (\%TR) and three-dimensional helical axes of motion (HAM) patterns of the C5-C6 MSU.

The kinetic analysis involved a comparison of the average global rotational stiffness (aGRS), the compressive disc forces at C5-C6 (CDF), and the compressive left or right facet forces at C5C6 (CLFF or CRFF). The aGRS was calculated at the point of maximum applied load. The disc and facet joint forces were animated using color-coded arrows representing vectors.

\subsection{Results}

\subsubsection{Distribution of relative MSU rotations}

The distributions of the percent contribution of rotations relative to the total rotation (\%TR) of the intact and implanted spine conditions during flexion, extension, left lateral bending, and right axial rotation are shown in Figure 5.2. The \%TR at the C5-C6 MSU of the intact spine was $22 \%$ in flexion, $21.9 \%$ in extension, $15.9 \%$ in lateral bending, and $21.3 \%$ in axial rotation.

Minimal changes occurred during flexion and lateral bending for all three disc designs. During extension, the \%TR of the C5-C6 MSU was 15.1\% for PDD-I, 24.3\% for PDD-II, and 24.7\% for 
(A)

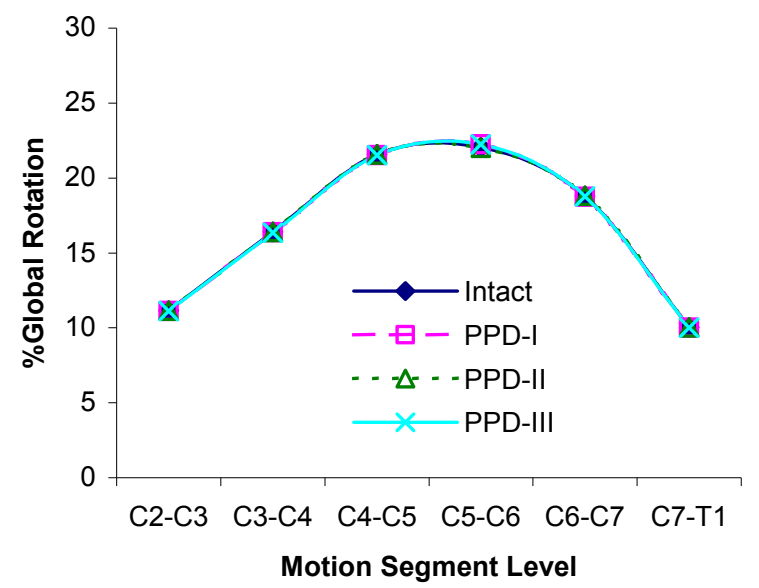

(C)

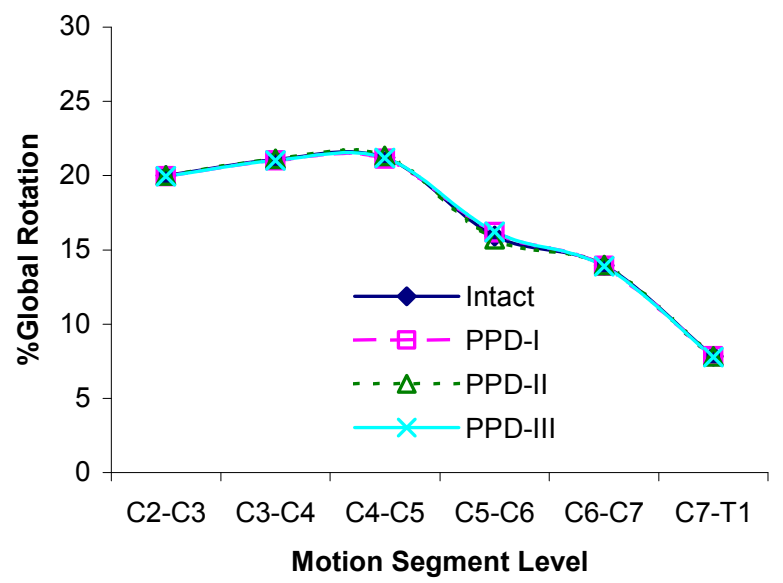

(B)

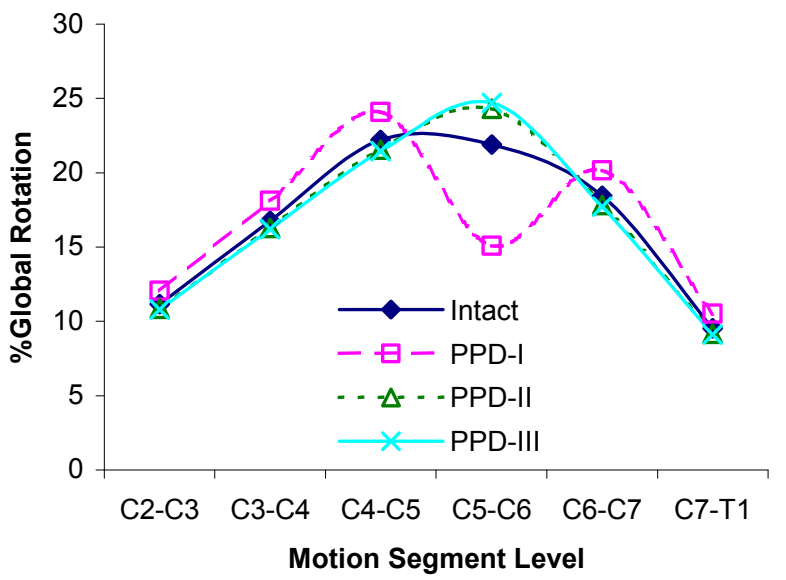

(D)

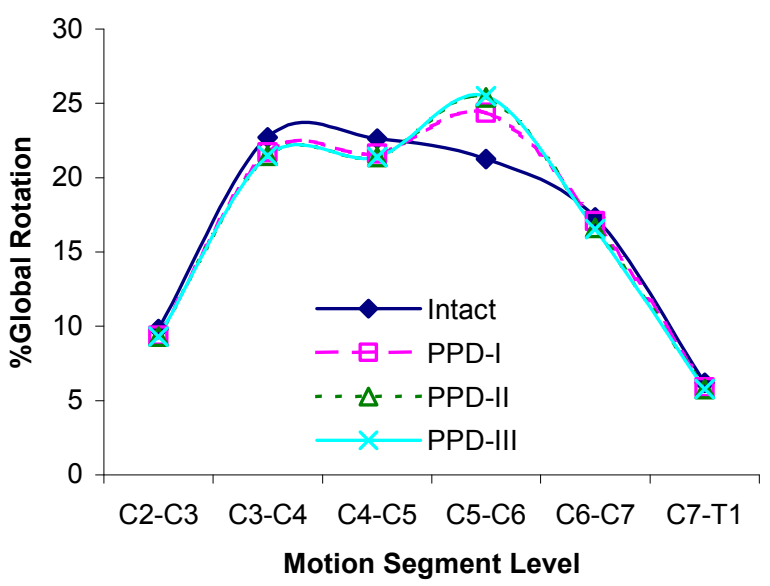

Figure 5.2 Relative MSU contributions for the intact spine and prosthetic disc designs

(PDD-I, II, and III). (A) Flexion, (B) Extension, (C) Left lateral bending, and (D) Right axial rotation. The individual motion segment unit rotations were expressed as percentages of overall global rotation. 
PDD-III. During axial rotation, the \%TR of the C5-C6 MSU was 24.3\% for PDD-I, 25.4\% for

PDD-II, and $25.5 \%$ for PDD-III.

\subsubsection{Helical axis of motion patterns}

Virtual animations of the computed HAM at C5-C6 for flexion, extension, lateral bending, and axial rotation of the intact spine, and three disc designs (PDD-I, PDD-II, and PDD-III) are shown in Figure 5.3. Black, red, blue, and cyan arrows illustrated the HAM between C5-C6 for the intact spine, PDD-I, PDD-II, and PDD-III, respectively. During flexion, the average position of the HAM for the intact spine passed near the upper endplate of C6. The HAM of PDD-III also passed near the endplate of C6. The HAM of PDD-I and PDD-II passed through the center of the spherical joint at the mid-region $\mathrm{C} 5-\mathrm{C} 6$ disc and $6.5 \mathrm{~mm}$ below the mid-region of the $\mathrm{C} 5$ C6 disc, respectively. During extension, the average position of the HAM for the intact spine passed through the mid-region of the C6 vertebral body. The HAM of PDD-II and PDD-III passed through similar locations of the $\mathrm{C} 6$ vertebral body. The HAM of PDD-I passed through the center of the spherical joint at the mid-height of the C5-C6 disc.

During lateral bending, the HAM for the intact spine passed obliquely through the upper cervical vertebrae (C5) in superior and posterior direction. This agreed with the prediction of Penning and Wilmink (1987). The HAM of PDD-III passed through a similar location of the 

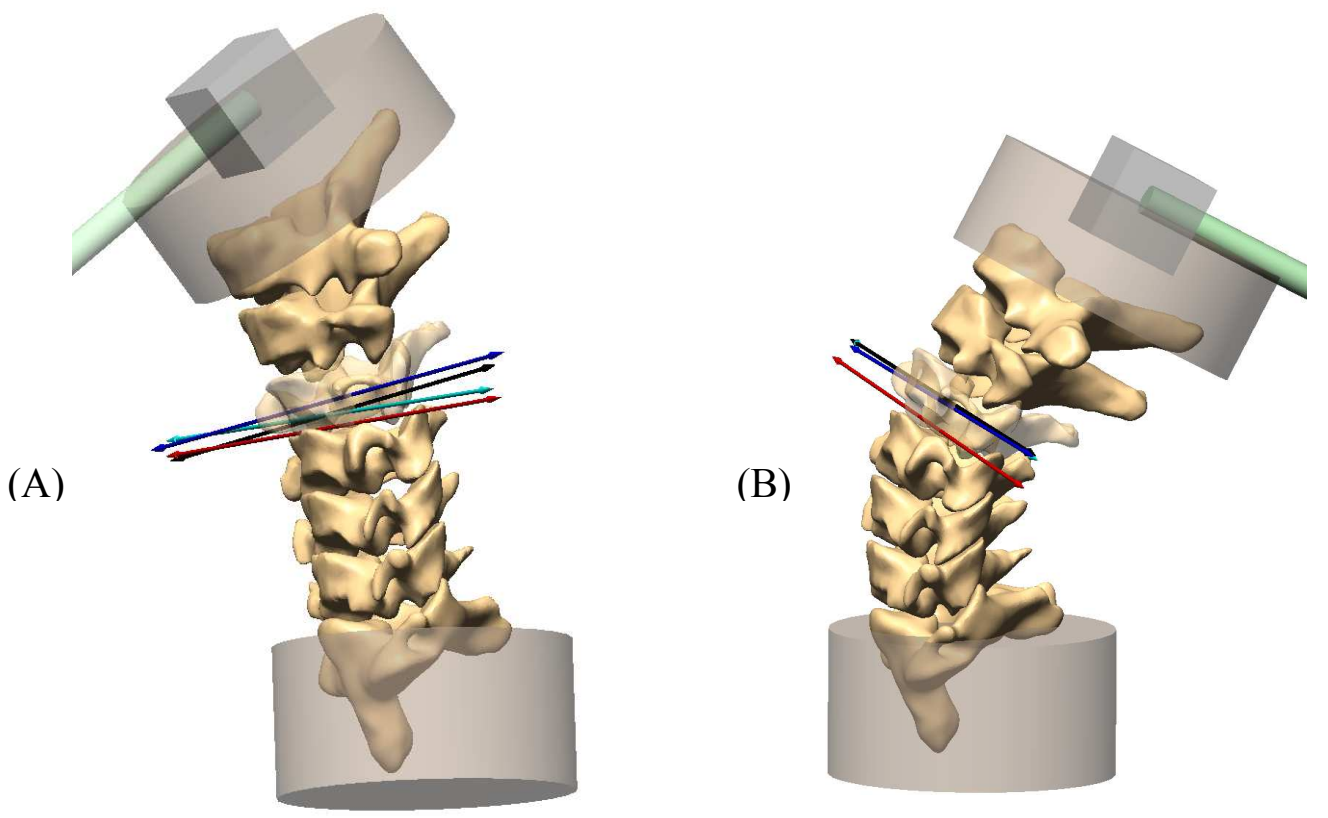

(C)
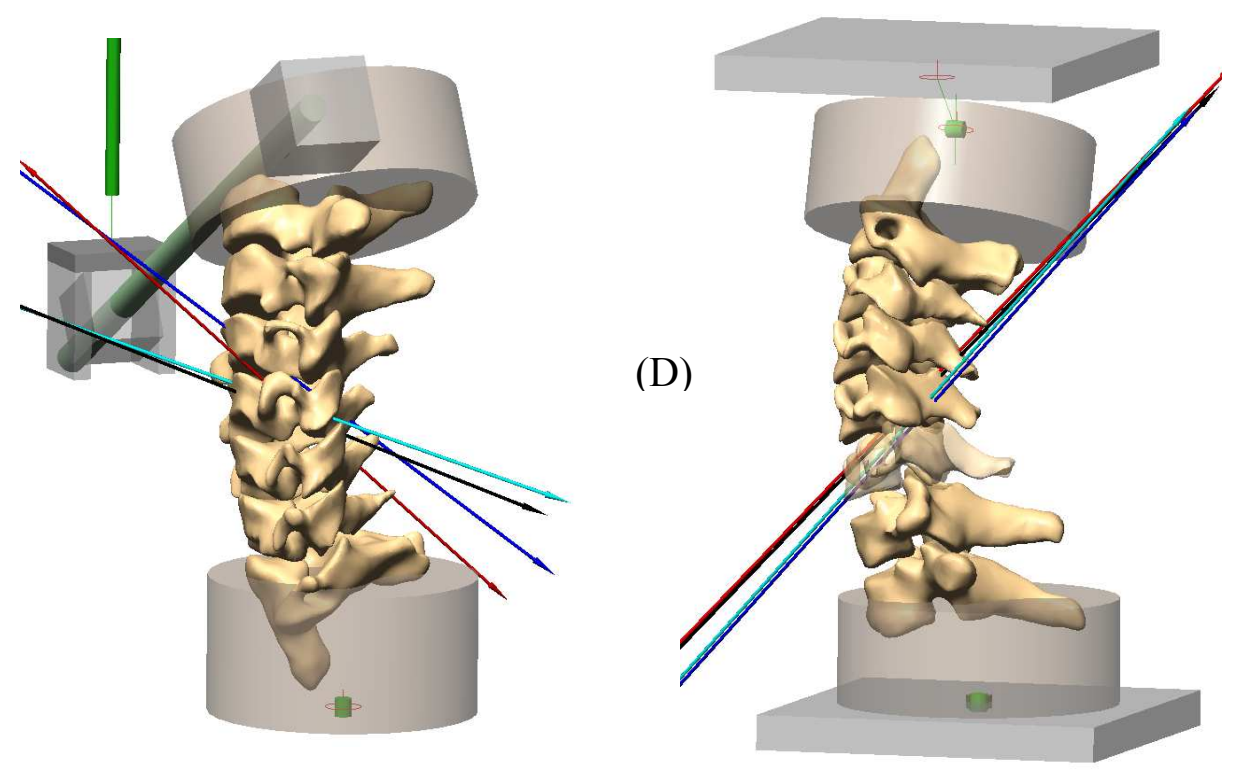

Figure 5.3 Animation frames of the HAM. (A) Flexion, (B) Extension, (C) Left lateral bending, and (D) Right axial rotation. The HAM rendered for each frame was the averaged HAM of the entire history of C5-C6 motion. The arrows illustrate HAM between C5-C6: Black arrow $=$ HAM of intact spine; Red arrow $=$ HAM of PDD-I; Blue arrow $=$ HAM of PDD-II; Cyan arrow $=$ HAM of PDD-III. 
C5 vertebral body as the HAM of the intact spine. The HAM of PDD-I and PDD-II passed through the center of the spherical joint at the mid-height of the C5-C6 disc and $6.5 \mathrm{~mm}$ below the mid-level of the C5-C6 disc, respectively.

During axial rotation, the HAM for the intact spine passed through the mid-region of the C5C6 disc. The HAM of PDD-I passed through the center of the spherical joint, which is also the mid disc. The HAM of PDD-II and PDD-III passed through the mid-region of the vertebral body of C6.

\subsubsection{Global rotational stiffness}

The global stiffness (applied moment versus global rotation) curves of the four spine conditions for flexion, extension, left lateral bending, and right axial rotation loading are shown in Figure 5.4. The average global rotational stiffness (aGRS) for the intact spine in flexion, extension, lateral bending, and axial rotation were $0.11,0.09,0.11$, and $0.09 \mathrm{Nm} / \mathrm{deg}$, respectively. During flexion, lateral bending, and axial rotation, negligible changes occurred for all prosthetic disc designs. During extension, the average global rotational stiffness of PDD-I, PDD-II, and PDD-III was $0.13,0.07$, and $0.07 \mathrm{Nm} / \mathrm{deg}$, respectively. 
(A)

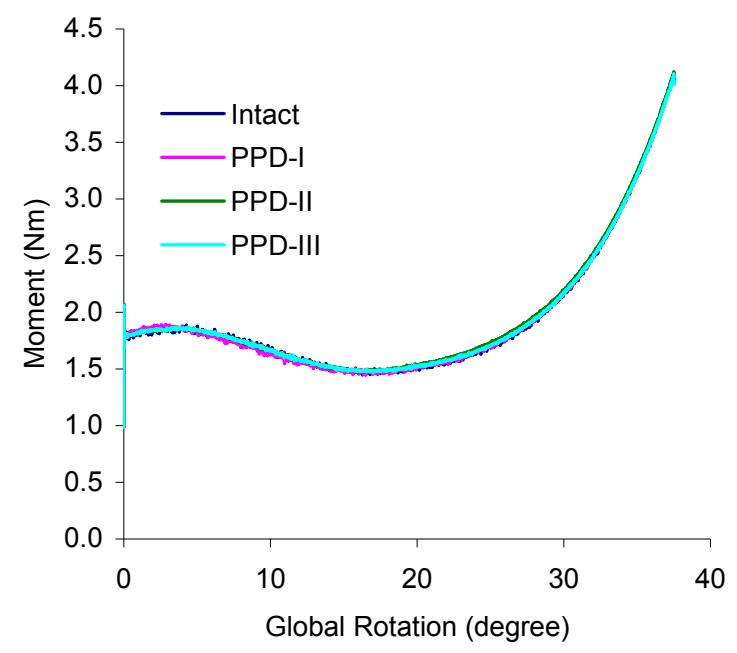

(C)

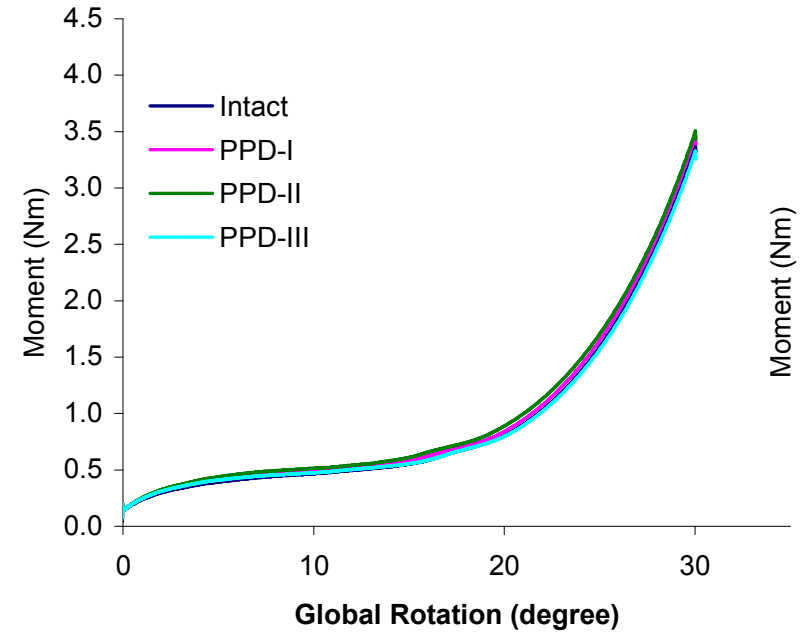

(B)

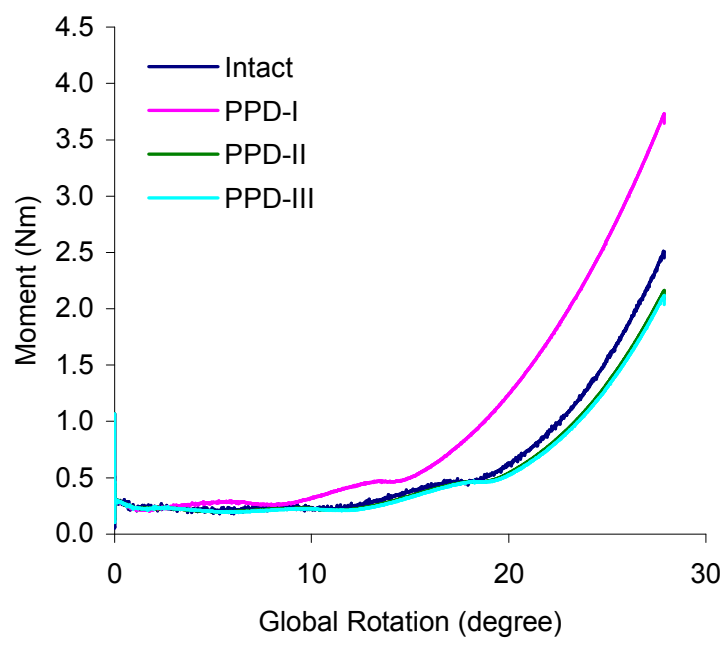

(D)

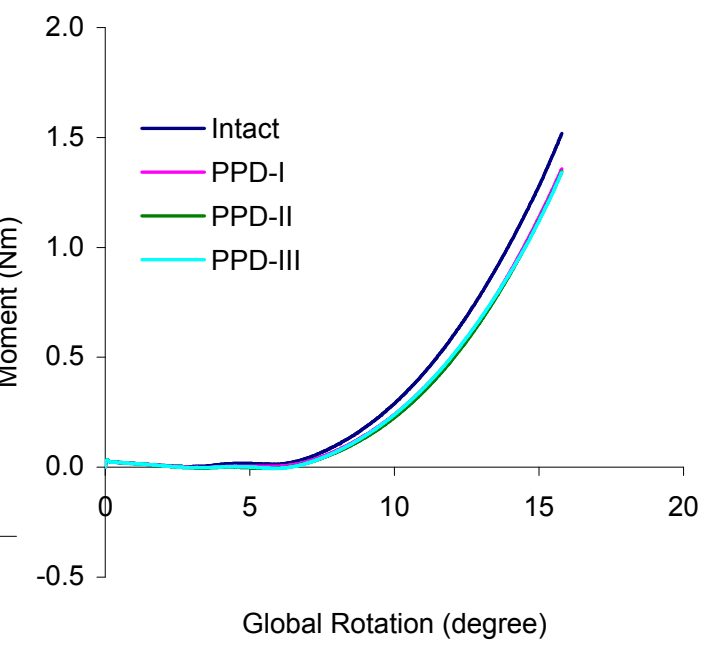

Figure 5.4 The applied moment versus global rotation curves. (A) Flexion, (B) Extension, (C) Left lateral bending, and (D) Right axial rotation. 


\subsubsection{Disc and facet joint forces}

The magnitude of disc and facet forces at the C5-C6 disc joint are provided in Table 5.1.

During flexion, the C5-C6 MSU disc force ranged between 96 and 146N amongst the three

prosthetic disc designs. During extension, the disc forces increased 18 times that of the intact

spine for PDD-I and 4 times for PDD-II. During lateral bending and axial rotation, minimal

changes occurred in the disc and facet forces. The compressive left and right facet forces (CLFF

and CRFF) at the C5-C6 MSU relative to the overall global rotation are shown in Figure 5.5 for

flexion, extension, left lateral bending, and right axial rotation. The facet forces of the intact

spine at the end limit of rotation were $0.9 \mathrm{~N}-\mathrm{CLFF}$ (tension) during flexion, $14.3 \mathrm{~N}-\mathrm{CLFF}$ during

extension 51.5N-CLFF and 17.1N-CRFF during lateral bending, and 1.3N-CLFF and 7.4N-

CRFF during axial rotation.

Table 5.1 Disc forces at C5-C6 (N).

\begin{tabular}{ccccc}
\hline & Intact & PDD-I & PDD-II & PDD-III \\
\hline Flexion & 95.6 & 124.5 & 145.9 & 103.1 \\
Extension & 38.1 & 691.4 & 160.0 & 18.0 \\
Lateral Bending & 28.8 & 37.0 & 62.1 & 1.8 \\
Axial Rotation & 28.1 & 23.1 & 14.2 & 14.7 \\
\hline
\end{tabular}



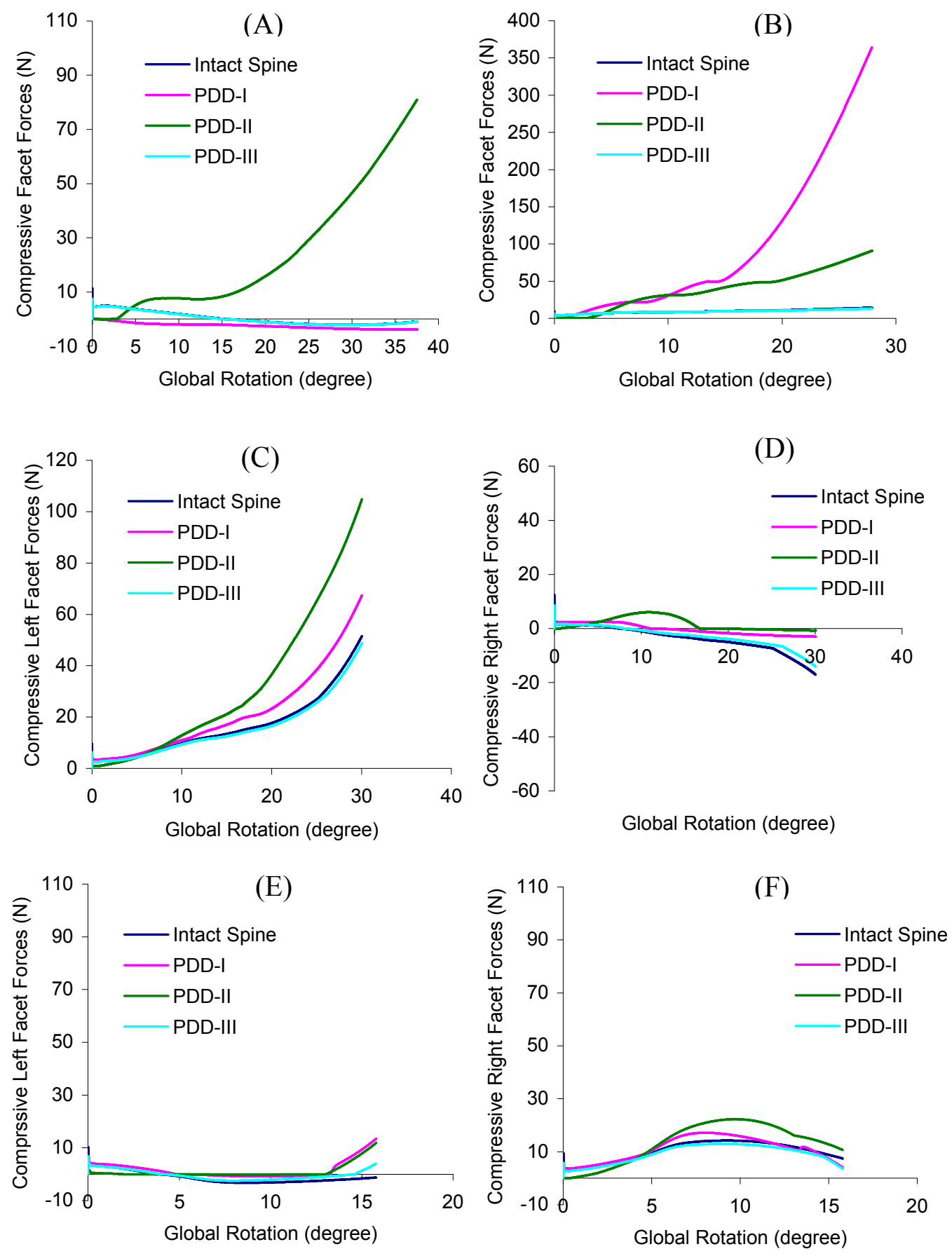

Figure 5.5 The facet forces (FF) at the C5-C6 MSU. (A) Left FF during flexion, (B) Left FF during extension, (C) Left FF during left lateral bending, (D) Right FF during left lateral bending. (E) Left FF during right axial rotation, and (F) Right FF during right axial rotation. 
At the end limit of flexion, PDD-I and PDD-III resulted in negligible changes, but the facet loads for PDD-II increased to $81 \mathrm{~N}-\mathrm{CLFF}$. At the end limit of extension, the facet loads increased to 364.4N-CLFF for PDD-I and 91N-CLFF for PDD-II. At the end limit of lateral bending the only significant facet forces occurred with PDD-I, 104.9N-CLFF. Minimal changes occurred in the facet forces during axial rotation.

\subsubsection{Animation of force vectors}

Animations of the force vectors (disc and facet joints) were performed for the intact spine and all three prosthetic disc designs. During flexion, the disc force was directed anteriorly for PDDII (Figure 5.6). During extension, the direction of disc joint force vectors changed upwardly and anteriorly for PDD-I and PDD-II (Figure 5.7). During lateral bending, the directions of force vectors of the C5-C6 disc joint changed to the anterior direction in PDD-I and PDD-II (Figure 5.8). During axial rotation, the directions of force vectors of the $\mathrm{C} 5-\mathrm{C} 6$ disc joint changed to the anterior direction in PDD-I (Figure 5.9). 


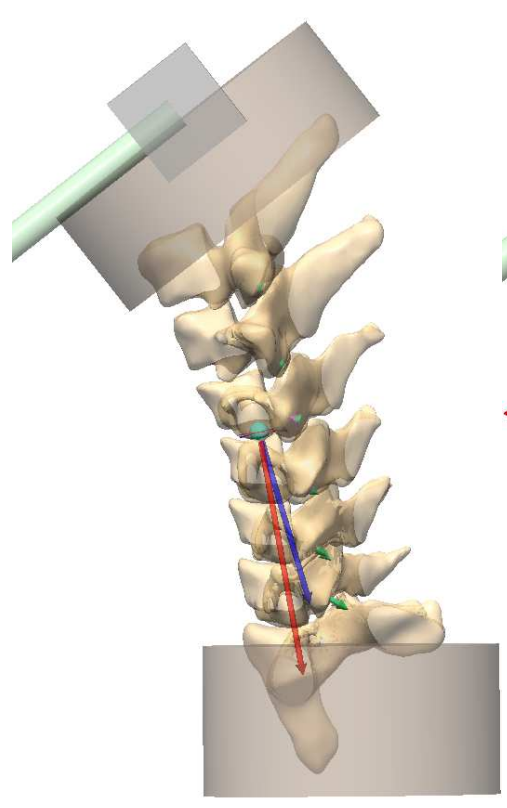

(A)

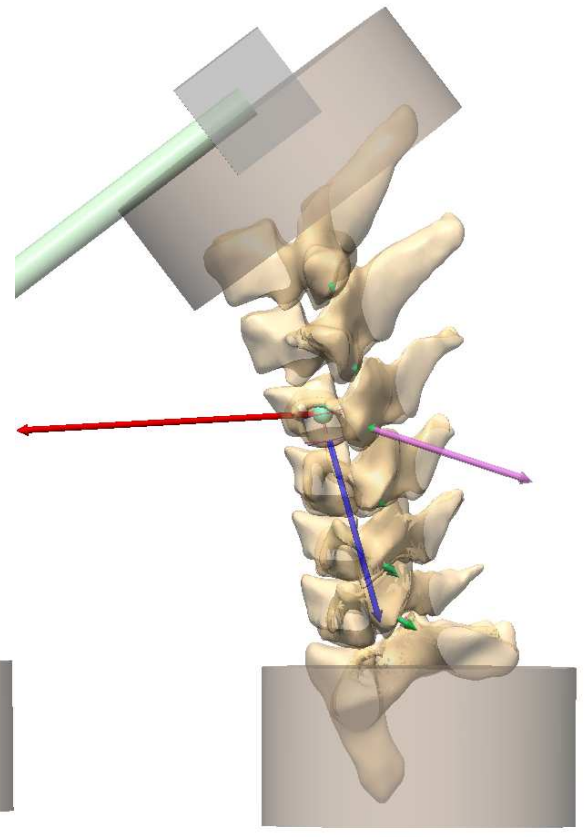

(B)

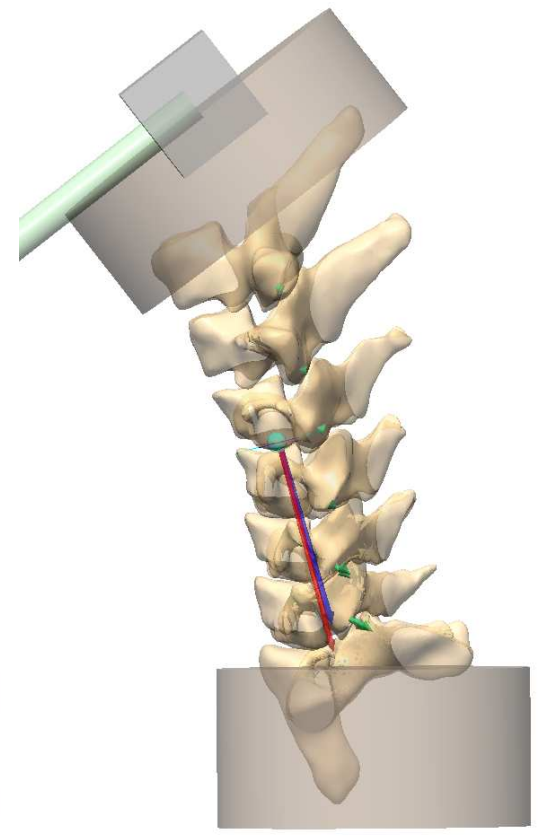

(C)

Figure 5.6 Animation frames of the disc and facet joint force vectors at C5-C6 MSU for intact and implanted spines during flexion. (A) PDD-I, (B) PPD-II, and (C) PPD-III. The arrows illustrate the force vectors: Blue arrow $=$ disc force of intact spine; Red arrow $=$ prosthetic disc force; Green arrow $=$ facet force of intact spine; Pink arrow $=$ facet force of prosthetic spine. 


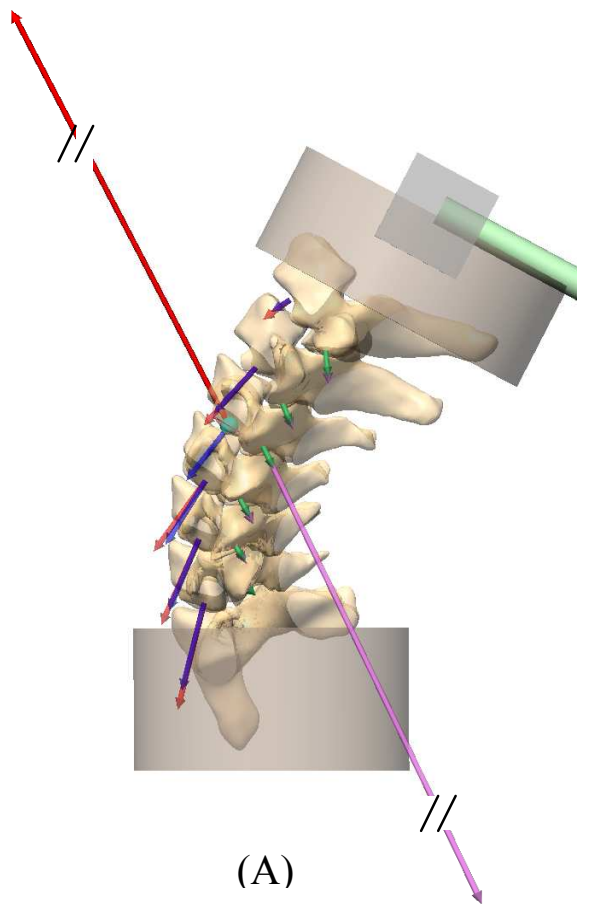

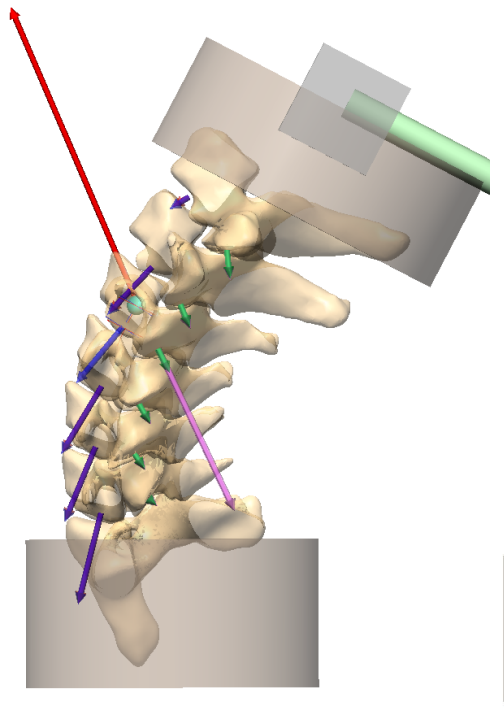

(B)

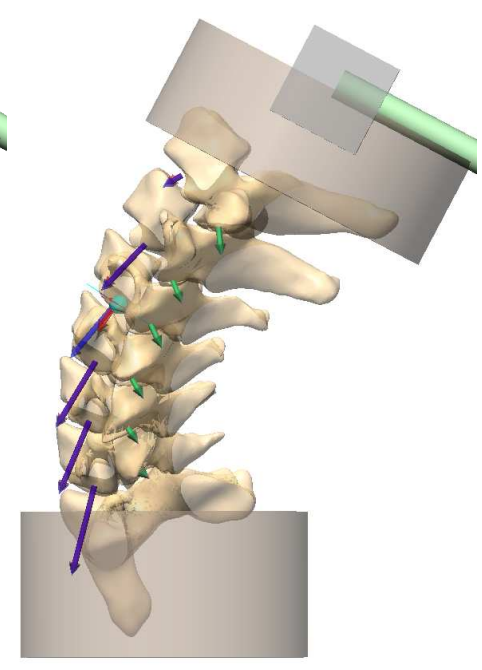

(C)

Figure 5.7 Animation frames of the disc and facet joint force vectors at C5-C6 MSU for intact and implanted spines during extension. (A) PDD-I, (B) PPD-II, and (C) PPD-III. The arrows illustrate the force vectors: Blue arrow $=$ disc force of intact spine; Red arrow $=$ prosthetic disc force; Green arrow $=$ facet force of intact spine; Pink arrow $=$ facet force of prosthetic spine. 


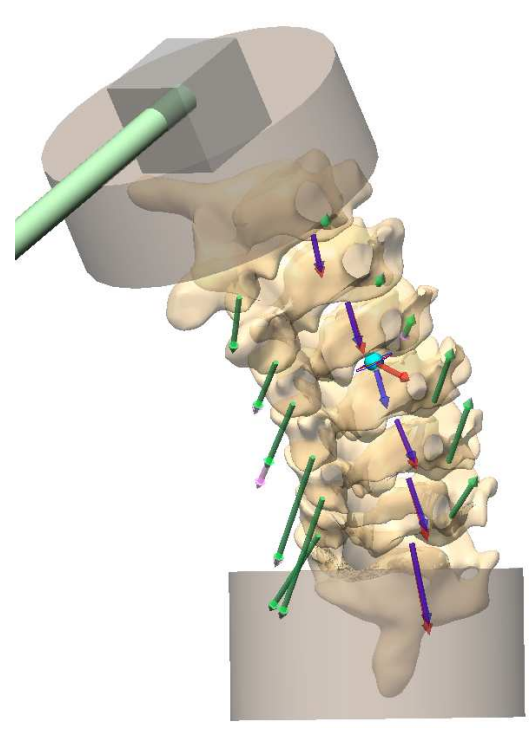

(A)

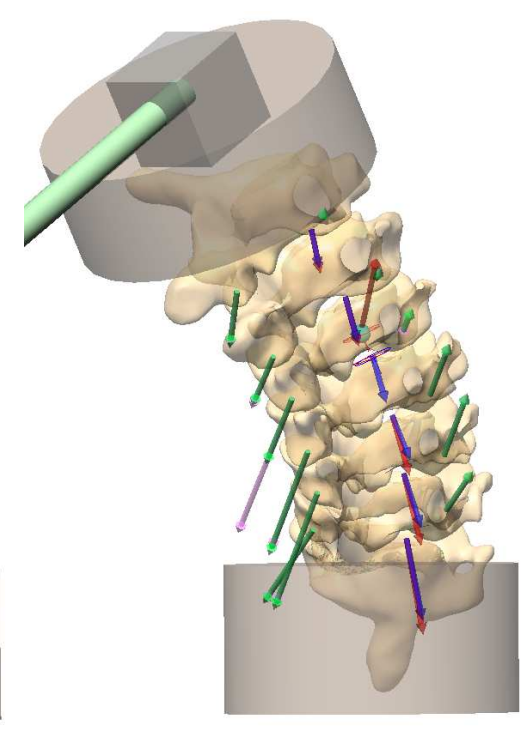

(B)

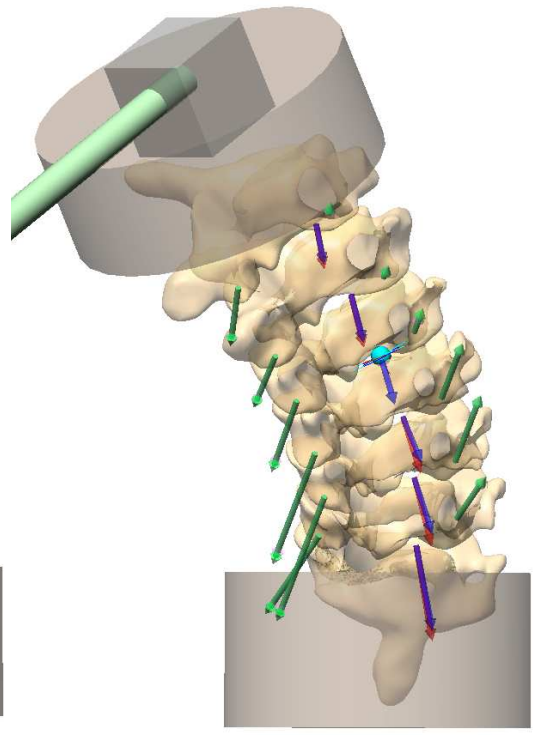

(C)

Figure 5.8 Animation frames of the disc and facet joint force vectors at C5-C6 MSU for intact and implanted spines during lateral bending. (A) PDD-I, (B) PPD-II, and (C) PPD-III. The arrows illustrate the force vectors: Blue arrow $=$ disc force of intact spine; Red arrow $=$ prosthetic disc force; Green arrow $=$ facet force of intact spine; Pink arrow $=$ facet force of prosthetic spine. 


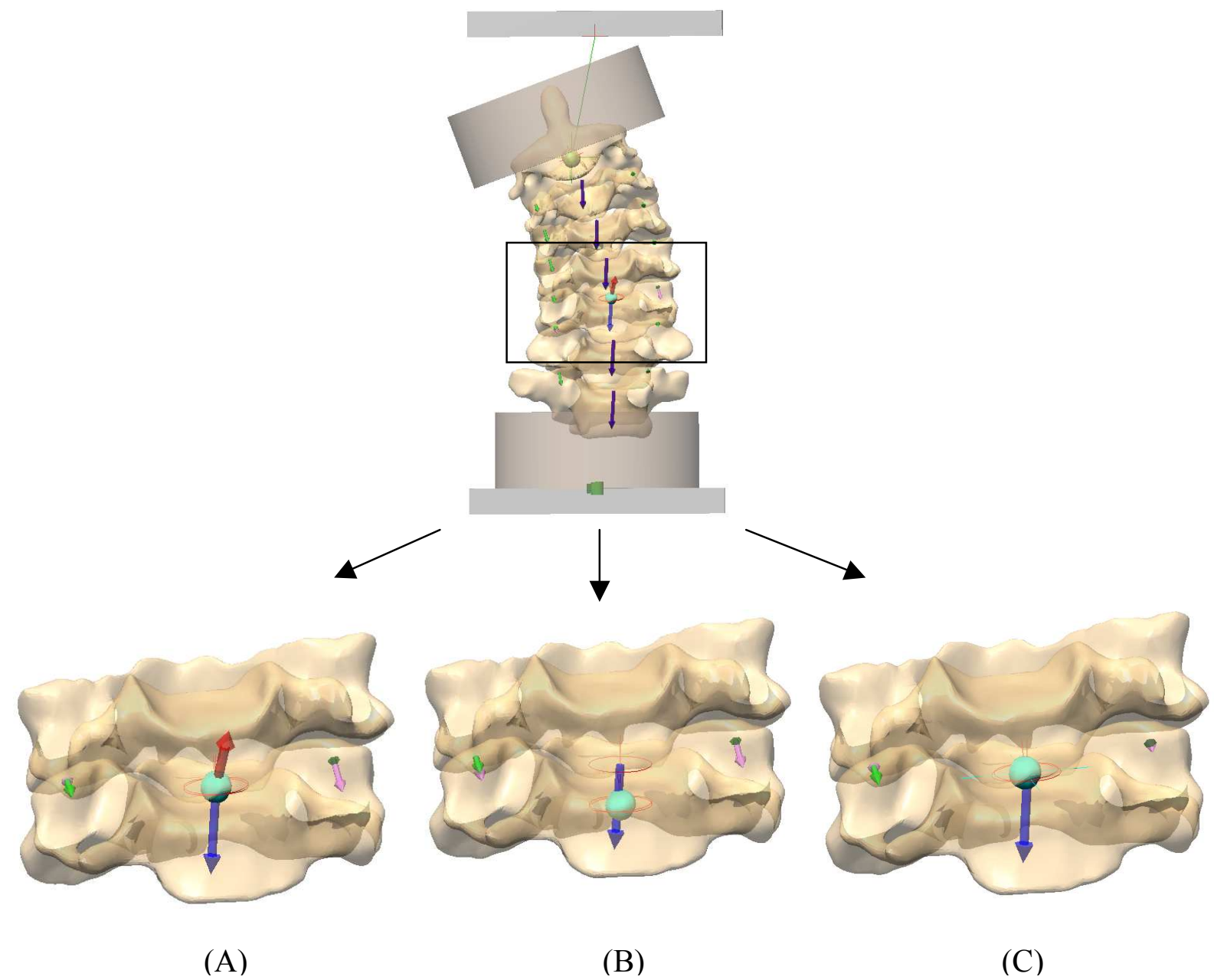

Figure 5.9 Animation frames of the disc and facet joint force vectors at C5-C6 MSU for intact and implanted spines during axial rotation. (A) PDD-I, (B) PPD-II, and (C) PPD-III. The arrows illustrate the force vectors: Blue arrow $=$ disc force of intact spine; Red arrow $=$ prosthetic disc force; Green arrow $=$ facet force of intact spine; Pink arrow $=$ facet force of prosthetic spine. 


\subsection{Discussion}

Disc arthroplasty provides an alternative approach to cervical fusion surgery for restoring and maintaining motion at a diseased spinal segment. When treating a diseased segment with a disc replacement, the normal kinematics and kinetics are restored by controlling the placement of the device and motion permitted (i.e., degrees of freedom). Currently, different paradigms exist for designing a cervical disc arthroplasty. In the present study, a virtual simulator of a cervical spine in vitro testing protocol was used to study the mechanics of various prosthetic disc design features. The simulation model served as a powerful design tool for better understanding the impact each simple design change may have on the overall function and dynamics of the disc.

\subsubsection{Flexion}

The measured parameters of the intact spine during flexion testing were aGRS: $0.11 \mathrm{Nm} / \mathrm{deg}$, \%TR: 22\%, CDF: $95.6 \mathrm{~N}$, and CLFF: $1 \mathrm{~N}$. The only notable changes amongst the different disc designs during flexion were the increased disc (145.9N) and facet (81N) forces for PDD-II. The HAM of the intact C5-C6 MSU was located near the upper endplate of the C6 vertebra. For PDD-II, the HAM passed through the subjacent body, $6.5 \mathrm{~mm}$ below the disc level. This difference might affect the kinematics of the spine and provoke facet impingement. During 
flexion of the intact spine, the facet joints of C5-C6 were under tension load, and a smaller arc of rotation was more beneficial than a larger arc of rotation. Thus, during flexion, a constrained joint such as PDD-I, located at the mid C5-C6 disc, works as well as a semi-constrained joint such as PDD-III.

\subsubsection{Extension}

During extension, a ball-and-socket device (PDD-I) constrained to the mid-disc region caused a significant increase in the disc and facet forces: $691.4 \mathrm{~N}-\mathrm{CDF}$ and 364.4N-CLFF compared to 38.1N-CDF and 14.3N-CLFF for the intact spine. Lowering the rotational axis into the subjacent body (PDD-II) caused a marginal increase in the disc and facet forces (160N-CDF and 91NCLFF). Further, un-constraining a ball-and-socket joint to a plane (PDD-III), resulted in the least deviations from the intact spine response. In terms of replicating the extensional HAM behavior, a constrained rotational device located in the subjacent body (PDD-II) was preferable over a rotational device placed at the mid-disc height (PDD-I). This was corroborated by lower facet force build-up for PDD-II compared to PDD-I. 


\subsubsection{Lateral bending}

In left lateral bending, the measured parameters for the intact spine were aGRS: $0.11 \mathrm{Nm} / \mathrm{deg}$, \%TR: 15.9, CDF: $28.8 \mathrm{~N}$, and CLFF: $51.5 \mathrm{~N}$ compression and CRFF: $17.1 \mathrm{~N}$ tension. A constrained ball-and-socket type joint introduced either a shearing component to the compressive disc force (anterior force vector for PDD-I) or increased the facet force (104.9N-CLFF and 0.8NCRFF-tension for PDD-II). During left lateral rotation, the left-sided facets were always in compression and the right-sided facets in tension. This compression and tension pattern was also present for all three disc designs. The HAM for PDD-III was located and oriented similar to that of the intact spine: in the upper part of the C5 vertebral body. However, the HAMs of PDD-I and PDD-II were located at or below the disc level, which resulted in increased facet loads. Never-the-less, the relative increase in the disc and facet forces during lateral bending was less than that which occurred during extension.

\subsubsection{Axial rotation}

During right axial rotation, the measured parameters of the intact spine were aGRS:

0.09Nm/deg, \%TR: $21.3 \%$, CDF: $28.1 \mathrm{~N}$, and CRFF: $1.3 \mathrm{~N}$ compression and CRFF: $7.4 \mathrm{~N}$ tension. For all disc scenarios, the \%TR increased to $25 \%$; negligible changes occurred in aGRS, CDF, and CLFF/CRFF except for an anterior-upward direction of CDF with PDD-I. During right axial 
rotation, the right-sided facets were always in compression and the left-sided facets in tension.

The level of loading was significantly less than that present during lateral bending. Although

the facet and disc loads did not change during axial rotation, the compression and tension pattern

was not maintained after the implantation of all three types of discs. Both facet joints were under

compression. This change of facet load pattern implies that some unconstrained vertical

translation of the spherical joint may be required to provide physiologic movements during axial rotation.

\subsubsection{Limitations}

This simulation model is not without limitations. A multi-body dynamic approach was used to study disc arthroplasty mechanics. Although this technique has advantages over FEM for dynamic analyses, this study does not have the ability to predict the changes of internal stress distributions. The changes in the forces were assumed to correlate with the changes in stresses in the tissue. The disc prosthesis was kinematically modeled as a spherical joint. In some situations, the prosthetic joint may have contacting surfaces that separate; however this type of movement was not included in this simulation. To accommodate for the changes in the tissue properties due to the surgical procedure itself, the stiffness properties were modified using experimental data from studies of the ProDisc-C prosthesis (DiAngelo et al., 2004). The surgical 
step of cutting through the ALL was simulated by appropriately reducing the material properties

of the intervertebral disc. Further, the ALL spans multiple vertebral bodies, but in the simulation model, the ALL was only connected to two adjacent vertebrae. The effects of such ligaments that span multiple bodies could not be included.

\subsubsection{Conclusions}

A constrained spherical joint placed at the disc level (PDD-I) significantly increased facet loads during extension. Lowering the rotational axis of a spherical joint towards the subjacent body (PDD-II) also caused a marginal increase in facet loading during flexion, extension, and lateral bending. However, un-constraining the spherical joint to a movement in a plane (PDDIII) minimized build up of facet and disc loads for all loading modes. Hopefully this study will provide clinicians and researchers with a theoretical idea to help understand artificial disc mechanics in a more intuitive and visual way. 


\section{Chapter 6}

\section{Discussion}

This chapter summarizes the major findings of this study. Section 6.1 discusses the technique for the development of a virtual model of the sub-axial cervical spine and evaluation processes of the model. Section 6.2 discusses the use of the model in the improvement of experimental protocols. Section 6.3 and Section 6.4 discuss the use of the model in the simulation study of single-level fusion and cervical disc arthroplasty, respectively. To improve the current model, limitations and recommendations are given in Section 6.5. Lastly, Section 6.6 gives general conclusions of this dissertation.

\subsection{Development and evaluation processes of the virtual model}

An accurate virtual model of the human sub-axial cervical spine was developed and used to simulate the in vitro testing protocol of our Biomechanics Laboratory. The bone geometry of the model was created from axial computerized tomography images. The joints linking adjacent vertebrae were modeled as a 'triple joint complex' comprised of: intervertebral disc, two facet joints, and surrounding ligaments. The intervertebral discs were modeled as non-linear 
viscoelastic elements (6 degree-of-freedom). The behaviors of the facet joints were approximated by a frictionless joint with a non-linear viscoelastic tension-compression component. Ligaments were modeled as non-linear spring-damper elements. A virtual laboratory simulation of our in vitro testing protocol was performed to evaluate the model response during flexion, extension, lateral bending, and axial rotation loading.

For kinematic evaluation, percent contribution of rotation relative to global rotation, coupling behaviors, and three-dimensional helical axes of motion (HAM) were analyzed. The results were consistent with the findings of our in vitro testing (Schwab, 2003) and data from the literature (Amevo et al., 1991; Milne, 1993; Panjabi et al., 2001; White and Panjabi, 1990). For kinetic evaluation, global rotational stiffness curves from the simulation were compared to and tested statistically with the data from in vitro testing (Schwab, 2003). Finally, the load vectors from the intervertebral discs and facet joints in each segment were calculated and visually animated to see how reasonable the individual level response was in reaction to a globally applied external load. This methodology produced realistic visualizations of in vitro experimentation and permitted analysis of the kinematics and kinetics of the cervical spine. With graphical illustration and animation features, this modeling technique provided the experimenter vivid and intuitive information. 


\subsection{A simulation study of axial rotational testing protocol}

Different mounting configurations for axial rotation testing of the cervical spine were studied to identify a set of end constraints that produced a physiologic motion response. A virtual multibody simulation model of the sub-axial cervical spine and an in vitro study utilizing a new testing system were performed to study the effects of three different end mounting constraints. The original Type 1 end mounting configuration that only allowed vertical translation produced non-physiologic kinematic and kinetic responses: opposite directions of coupling, anteriorposteriorly directed helical axes of motion (HAM) at the $\mathrm{C} 2-\mathrm{C} 3$ segment, the greatest global rotational stiffness for the different end constraints studied, and primarily shear forces at the disc joints of upper segments (C2-C3, $\mathrm{C} 3-\mathrm{C} 4$, and $\mathrm{C} 4-\mathrm{C} 5)$. Un-constraining the end mounts resulted in a physiologic motion response. The Type 2 (allowing vertical translation and lateral tilt) and

Type 3 (allowing vertical translation, lateral tilt, and lateral translation) mounting conditions produced a physiologic coupling behavior, physiologic HAMs, and reduced global rotational stiffness. In vitro testing produced a similar pattern of coupling behavior and global rotational stiffness compared to the simulation results. The use of the computer simulation demonstrated the current problems with pure axial rotation in vitro testing protocol through the three- 
dimensional kinematic and kinetic analysis of the virtual model and enabled efficient

modification to the in vitro testing protocol.

\subsection{A simulation study of single-level spine fusion}

The virtual model was used to study the biomechanical effects after a single-level fusion between C5-C6. The virtual dynamic model simulated flexion, extension, lateral bending, and axial rotation motion of sub-axial cervical spine in the intact and fused (at C5-C6) conditions.

The simulation's boundary conditions were modeled from our in vitro testing protocol (Schwab, 2003). Displacement control methods were used to apply a moment distribution across the cervical spine. The fusion at the C5-C6 level was modeled as a rigid joint (no motion) between C5 and C6, which is an idealistic model of fusion. The single-level fusion was found to produce increased motion compensation at the adjacent segments during flexion and extension. The global rotational stiffness was markedly increased after single-level fusion during all four modes

of loading. Greater increases in the disc forces were found in the level superior to the fusion (C4C5) during flexion and inferior to the fusion (C6-C7) during extension. The virtual simulation model suggests that eliminating motion at one segment leads to increased motion as well as to increased global stiffness and disc forces at the unfused segments. 


\subsection{A simulation study of cervical disc arthroplasty}

The virtual model of the sub-axial cervical spine was used to study three different prosthetic disc designs (PDD): 1) PDD-I: a 3 degree-of-freedom (DOF) spherical joint located at the mid C5-C6 disc, 2) PDD-II: a 3-DOF spherical joint located 6.5mm below the mid C5-C6 disc, and 3) PDD-III: a 5-DOF spherical joint in plane parallel with the C5-C6 disc level. A constrained spherical joint placed at the disc level (PDD-I) significantly increased facet loads during extension. Lowering the rotational axis of the spherical joint towards the subjacent body (PDDII) caused a marginal increase in facet loading during flexion, extension, and lateral bending. Lastly, un-constraining the spherical joint to move freely in a plane (PDD-III) minimized facet load build up during all loading modes. The simulation model served as a powerful design tool for better understanding the impact simple design changes may have on cervical disc dynamics.

\subsection{Limitations and recommendations}

To further improve the current model, the limitations and recommendations for possible

enhancements are given. Rotational stiffness properties of the intervertebral discs were derived primarily from lumped in vitro stiffness data, which included the contribution of surrounding tissues. Since ligaments and facet joints were also added to the model, the overall stiffness 
properties were increased. Therefore, final adjustments of ligament (tensile), and disc (rotational) stiffness properties were performed using an iterative process that sought to match the model global stiffness response to that of an in vitro data set. Although this approach produced an accurate model response, the responses of the individual components might not be correct. It is recommended that experiments quantifying the mechanical characteristics of individual components of cervical motion segments be undertaken. These experiments can be performed using a serial cut of individual components using fresh cadaver specimens. With this improvement of the model, the injury mechanism of individual components can be simulated and the force data of the components could be calculated more accurately.

Since the translational stiffness properties for discs were derived from Panjabi et al. (1986) and Shea et al. (1991), the rotational and translational data for the discs were from different sources. To improve the model accuracy, the experiments to generate translational stiffness data should be performed using simulation testing protocols. Also recommended are dynamic experiments quantifying the viscous characteristics of intervertebral discs. Current publications do not give accurate damping coefficient values for the discs of the human cervical spine. The damping coefficient used in this model was not derived from in vitro testing, but from empirical experience producing appropriate model responses. 
This model was primarily developed to simulate loading conditions. To produce realistic simulations of the loading and unloading of cervical specimens, hysteresis properties should be added into the current material properties. One way to incorporate the hysteresis behaviors into the current model is to use separate material properties curves for the unloading condition. These curves can be easily generated using a scaling factor from original stiffness curves. To scale more accurately, the scaling factors might also be calculated from in vitro testing data. This is the concept of pseudo-elasticity, which is a drastic simplication of quasi-linear viscoelasticity (Fung, 1993). Using this method, it may be possible to simulate the biological responses for cyclic loading, creep, and relaxation.

Although ligaments in this model have failure properties, the disc and facet joints do not. For future opportunities of simulating spine injury, the failure properties should be included in the current model.

One limitation with modeling the facet joints was that these joints were not modeled as contacting surfaces, but rather as mechanical connections at a single defined point in space with specific stiffness properties. Tensile and compressive stiffness properties assigned to these joints were derived from the properties of the capsular ligament and intervertebral disc, respectively. The material properties of the capsular ligament could approximate the tensile properties of the facet joint. It was also assumed that the compressive properties of the intervertebral disc were 
the best approximation of the compressive properties of facet joint cartilage during the physiologic ranges of the loading condition. This assumption was a limitation of the model if the loading conditions were beyond the physiologic ranges. To the author's knowledge, there are no prior studies reporting compressive stiffness properties of facet joint cartilage. However the equilibrium modulus and dynamic stiffness data of articular cartilage from knee and ankle joints (Treppo et al., 2000) could have possibly been used for the properties of the facet joints.

Physiologically, ligaments of the spine span multiple segments. In our simulation model, the ligaments originated at one vertebral body and terminated at the adjacent vertebral body, spanning a single motion segment unit. Simulation of multiple segment spanning ligaments and their related effects could not be simulated. The current version of VisualNastran 4D does not provide any direct solution for this matter. A curved slot with multiple insertion points could be one solution for this problem. It would demand a significant amount of computing power, however. The viscoelastic behavior of ligaments was modeled using the Kelvin (standard linear solid) model. To accurately simulate the viscoelastic properties of biologic tissues, the Voight model would have been more appropriate. This Voight model would also increase the demand of computing power significantly. 
Lastly, a parametric study will be needed to determine the critical stiffness parameters and to more accurately identify the mechanical characteristics of the simulation model. The fractional factorial design or Taguchi's robust parameter design are recommended.

\subsection{Conclusions}

In conclusion, a virtual sub-axial cervical spine model and an experimental laboratory simulator were developed. This methodology produced realistic visualizations of in vitro experimentation and permitted analysis of the kinematics and kinetics of the cervical spine. With graphical illustration and animation features, this modeling technique provided the experimenter vivid and intuitive information. For further applications, the virtual cervical spine model was used to modify an in vitro testing protocol, and to study the mechanics of spine fusion and the mechanics of cervical disc arthroplasty. This virtual model will bridge the gap between the cadaveric-based in vitro tests and clinical-based experimental studies to further research efforts and educational knowledge of cervical spine biomechanics. The virtual spine model can be readily adapted to simulate other extrinsic factors, such as instabilities, fractures, trauma, or to study the influence of other spinal instrumentation systems. 


\section{Chapter 7}

\section{Suggested Future Work}

Because the facet joints of the sub-axial cervical spine have almost flat articular surfaces, the facet joint behaviors could be simulated in an efficient way in this model. However, the approach used for facet joints in the sub-axial spine was not sufficient for the occipital-atlantoaxial complex (C0-C1-C2). The lower facets of the atlas (C1) and upper facets of the axis (C2) are convex. In a neutral position, the convex surface of the lower facet cartilage of the atlas $(\mathrm{C} 1)$ rests on the convex surface of the upper facet cartilage of the axis (C2). During axial rotation the atlas descends and ascends as it slips along the slope of the cartilage (Bogduk and Mercer, 2000). Therefore, colliding biconvex surfaces to simulate this movement at C1-C2 should be developed. In order to further refine the accuracy of the model, some amount of overlap between colliding surfaces must be allowed. To further enhance the whole spine model, the convex occipital condyles of the skull, the concave upper facet joints of $\mathrm{C} 1$, and the odontoid process, and reciprocal contact areas in the atlas should be modeled as colliding surfaces. To accomplish accurate kinematic response using these colliding surfaces, balanced capsular ligament structures surrounding the joints should be added as well. 
For further development, muscle components should be included to simulate the in vivo active muscle motion response. Fortunately, the VisualNastran 4D program can be integrated into another control modality such as Simulink (The MathWorks, Natick, MA) that works in conjunction with a muscle simulator program named 'Virtual Muscle' (Cheng et al., 2000). The virtual muscle simulation has the functionality to produce different kinds of fiber types and muscles using muscle morphometry data from the literature and several muscle parameters. The sensory motor control strategy including EMG signals can be used to control the muscle responses. In the case of integration simulation with the Virtual Muscle, only the force-based linear actuator component in VisualNastran 4D is available for muscle modeling. The linear actuator component is a straight component that has no bending properties. Therefore, modeling muscles that follow the curvature of the spine need to be developed. One approach for curving the actuators to simulate the neck muscles is to connect multiple actuators together along the curved path. A problem with this technique is that the curved slot feature needs a great amount of computing time and the multiple actuator components would be shortened in different amounts and rates. A very sophisticated control strategy would also be required to balance all of these components. 


\section{List of References}




\section{List of References}

Ackerman, M. J. and Banvard, R. A. (2000) Imaging outcomes from the National Library of Medicine's Visible Human Project. Computerized Medical Imaging \& Graphics 24, 125-126.

Amevo, B., Worth, D., and Bogduk, N. (1991) Instantaneous axes of rotation of the typical cervicalmotion segments: a study in normal volunteers. Clinical Biomechanics 6, 111-117.

Anderson, P. A. and Rouleau, J. P. (2004) Intervertebral disc arthroplasty. Spine 29, 2779-2786.

Arnold, A. S., Asakawa, D. J., and Delp, S. L. (2000) Do the hamstrings and adductors contribute to excessive internal rotation of the hip in persons with cerebral palsy? Gait and Posture 11, 181-190.

Baba, H., Furusawa, N., Imura, S., Kawahara, N., Tsuchiya, H., and Tomita, K. (1993) Late radiographic findings after anterior cervical fusion for spondylotic myeloradiculopathy. Spine 18, 2167-2173.

Beggs, J. S. (1983) Kinematics. Hemisphere Pub. Corp., Washington.

Beylot, P., Gingins, P., Kalra, P., Thalmann, N., Maurel, W., Thalmann, D., and Fasel, J. (1996) 3D interactive topological modeling using Visible Human dataset. Journal of the European Association for Computer Graphics 15, 33-44.

Bogduk, N. and Mercer, S. (2000) Biomechanics of the cervical spine. I: Normal kinematics. Clinical Biomechanics 15, 633-648.

Bogduk, N. and Yoganandan, N. (2001) Biomechanics of the cervical spine Part 3: minor injuries. Clinical Biomechanics 16, 267-275.

Braunstein, E. M., Hunter, L. Y., and Bailey, R. W. (1980) Long term radiographic changes following anterior cervical fusion. Clinical Radiology 31, 201-203.

Brodke, D. S. and Zdeblick, T. A. (1992) Modified Smith-Robinson procedure for anterior cervical discectomy and fusion. Spine 17, S427-S430.

Buttner-Janz, K., Schellnack, K., and Zippel, H. (1989) Biomechanics of the SB Charite lumbar intervertebral disc endoprosthesis. International Orthopedics 13, 173-176. 
Chao, E. Y. (2003) Graphic-based musculoskeletal model for biomechanical analyses and animation. Medical Engineering and Physics 25, 201-212.

Cheng, E. J., Brown, I. E., and Loeb, G. E. (2000) Virtual muscle: a computational approach to understanding the effects of muscle properties on motor control. Journal of Neuroscience Methods 101, 117-130.

Connolly, E. S., Seymour, R. J., and Adams, J. E. (1965) Clinical evaluation of anterior cervical fusion for degenerative cervical disc disease. Journal of Neurosurgery 23, 431-437.

Coutinho, M. G. (2001) Dynamic Simulations of Multibody Systems. Springer, New York.

Cripton, P. A., Sati, M., Orr, T. E., Bourquin, Y., Dumas, G. A., and Nolte, L. P. (2001)

Animation of in vitro biomechanical tests. Journal of Biomechanics 34, 1091-1096.

Cummins, B. H., Robertson, J. T., and Gill, S. S. (1998) Surgical experience with an implanted artificial cervical joint. Journal of Neurosurgery 88, 943-948.

Cunningham, B. W., Lowery, G. L., Serhan, H. A., Dmitriev, A. E., Orbegoso, C. M., McAfee, P. C., Fraser, R. D., Ross, R. E., and Kulkarni, S. S. (2002) Total disc replacement arthroplasty using the AcroFlex lumbar disc: a non-human primate model. European Spine Journal 11 Suppl 2, S115-S123.

De Jager, M. K. J. (1996) Mathematical head-neck models for acceleration impacts. PhD. Thesis, Technical University of Eindhoven.

Delp, S. L. and Loan, J. P. (1995) A graphics-based software system to develop and analyze models of musculoskeletal structures. Computers in Biology and Medicine 25, 21-34.

Delp, S. L. and Loan, .J. P. (2000) A computational framework for simulation and analysis of human and animal movement. IEEE Computing in Science and Engineering 2, 46-55.

Deng, Y. C. and Goldsmith, W. (1987) Response of a human head/neck/upper-torso replica to dynamic loading--II. Analytical/numerical model. Journal of Biomechanics 20, 487-497.

DiAngelo, D. J. and Foley, K. T. (2003) An improved biomechanical testing protocol for evaluating multilevel cervical instrumentation in a human cadaveric corpectomy model. In Spinal Implants: Are We Evaluating Them Appropriately? ASTM International, West Conshohocken. 
DiAngelo, D. J. and Foley, K. T. (2004) An improved biomechanical testing protocol for evaluating spinal arthroplasty and motion preservation devices in a multilevel human cadaveric cervical model. Neurosurgical Focus 17, 22-29.

DiAngelo, D. J., Foley, K. T., Morrow, B. R., Schwab, J. S., Song, J., German, J. W., and Blair, E. (2004) In vitro biomechanics of cervical disc arthroplasty with the ProDisc-C total disc implant. Neurosurgical Focus 17 (3):E7, 44-54.

DiAngelo, D. J., Foley, K. T., Vossel, K. A., Rampersaud, Y. R., and Jansen, T. H. (2000) Anterior cervical plating reverses load transfer through multilevel strut grafts. Spine 25, 783-795.

DiAngelo, D. J., Roberston, J. T., Metcalf, N. H., McVay, B. J., and Davis, R. C. (2003) Biomechanical testing of an artificial cervical joint and an anterior cervical plate. Journal of Spinal Disorders and Technique 16, 314-323.

Dooris, A. P., Goel, V. K., Grosland, N. M., Gilbertson, L. G., and Wilder, D. G. (2001) Loadsharing between anterior and posterior elements in a lumbar motion segment implanted with an artificial disc. Spine 26, E122-E129.

Eck, J. C., Humphreys, S. C., Lim, T. H., Jeong, S. T., Kim, J. G., Hodges, S. D., and An, H. S. (2002) Biomechanical study on the effect of cervical spine fusion on adjacent-level intradiscal pressure and segmental motion. Spine 27, 2431-2434.

Emery, S. E., Bolesta, M. J., Banks, M. A., and Jones, P. K. (1994) Robinson anterior cervical fusion comparison of the standard and modified techniques. Spine 19, 660-663.

Fagan, M. S., Julian, S. and Mohsen A. M. (2002) Finite element analysis in spine research. Proceedings of the Institution of Mechanical Engineers. Part H, Journal of Engineering in Medicine. 216, 281-298.

Foley, K. T., DiAngelo, D. J., Rampersaud, Y. R., Vossel, K. A., and Jansen, T. H. (1999) The in vitro effects of instrumentation on multilevel cervical strut-graft mechanics. Spine 24, 23662376.

Fuller, D. A., Kirkpatrick, J. S., Emery, S. E., Wilber, R. G., and Davy, D. T. (1998) A kinematic study of the cervical spine before and after segmental arthrodesis. Spine 23, 1649-1656.

Fung, Y. C. (1993) Biomechanics: Mechanical Properties of Living Tissues. Springer-Verlag, New York. 
García de Jalón, J. and Bayo, E. (1994) Kinematic and Dynamic Simulation of Multibody systems: the real-time challenge. Springer-Verlag, New York.

Gerritsen, K. G., Nachbauer, W., and van den Bogert, A. J. (1996) Computer simulation of landing movement in downhill skiing: anterior cruciate ligament injuries. Journal of Biomechanics 29, 845-854.

Goel, V. K. and Clausen, J. D. (1998) Prediction of load sharing among spinal components of a C5-C6 motion segment using the finite element approach. Spine 23, 684-691.

Goffin, J., Casey, A., Kehr, P., Liebig, K., Lind, B., Logroscino, C., Pointillart, V., Van Calenbergh, F., and van Loon, J. (2002) Preliminary clinical experience with the Bryan Cervical Disc Prosthesis. Neurosurgery 51, 840-845.

Goffin, J., Van Calenbergh, F., van Loon, J., Casey, A., Kehr, P., Liebig, K., Lind, B., Logroscino, C., Sgrambiglia, R., and Pointillart, V. (2003) Intermediate follow-up after treatment of degenerative disc disease with the Bryan Cervical Disc Prosthesis: single-level and bi-level. Spine 28, 2673-2678.

Gore, D. R. and Sepic, S. B. (1984) Anterior cervical fusion for degenerated or protruded discs. A review of one hundred forty-six patients. Spine 9, 667-671.

Green, P. W. (1977) Anterior cervical fusion. A review of thirty-three patients with cervical disc degeneration. Journal of Bone and Joint Surgery (British) 59, 236-240.

Gunzburg, R., Mayer, H. M., Szpalski, M., and Aebi, M. (2002) Arthroplasty of the spine: the long quest for mobility. European Spine Journal 11 Suppl 2, S63-S64.

Herkowitz, H. N., Kurz, L. T., and Overholt, D. P. (1990) Surgical management of cervical soft disc herniation. A comparison between the anterior and posterior approach. Spine 15, 1026-1030.

Hilibrand, A. S., Carlson, G. D., Palumbo, M. A., Jones, P. K., and Bohlman, H. H. (1999)

Radiculopathy and myelopathy at segments adjacent to the site of a previous anterior cervical arthrodesis. Journal of Bone and Joint Surgery (American) 81, 519-528.

Hilibrand, A. S., Yoo, J. U., Carlson, G. D., and Bohlman, H. H. (1997) The success of anterior cervical arthrodesis adjacent to a previous fusion. Spine 22, 1574-1579. 
Hunter, L. Y., Braunstein, E. M., and Bailey, R. W. (1980) Radiographic changes following anterior cervical fusion. Spine 5, 399-401.

Kumaresan, S., Yoganandan, N., and Pintar, F. A. (1997a) Finite element analysis of anterior cervical spine interbody fusion. Biomedical Materials and Engineering 7, 221-230.

Kumaresan, S., Yoganandan, N., and Pintar, F. A. (1998) Finite element modeling approaches of human cervical spine facet joint capsule. Journal of Biomechanics 31, 371-376.

Kumaresan, S., Yoganandan, N., and Pintar, F. A. (1999a) Finite element analysis of the cervical spine: a material property sensitivity study. Clinical Biomechanics 14, 41-53.

Kumaresan, S., Yoganandan, N., Pintar, F. A., and Maiman, D. J. (1999b) Finite element modeling of the cervical spine: role of intervertebral disc under axial and eccentric loads. Medical Engineering and Physics 21, 689-700.

Kumaresan, S., Yoganandan, N., Pintar, F. A., Voo, L. M., Cusick, J. F., and Larson, S. J. (1997b) Finite element modeling of cervical laminectomy with graded facetectomy. Journal of Spinal Disorders 10, 40-46.

Le, H., Thongtrangan, I., and Kim, D. H. (2004) Historical review of cervical arthroplasty. Neurosurgical Focus 17(3):E1, 1-9.

Lee, C. K. and Goel, V. K. (2004) Artificial disc prosthesis: design concepts and criteria. Spine Journal 4, 209S-218S.

Lindberg, L. (1970) Anterior cervical fusion for cervical rhizopathies. A follow-up study. Acta Orthopeadic Scandinavica 41, 312-319.

Link, H. D., McAfee, P. C., and Pimenta, L. (2004) Choosing a cervical disc replacement. Spine Journal 4, S294-S302.

Lysell E. (1969) Motion in the cervical spine: an experimental study on autopsy specimens. Acta Orthopaedica Scandinavica 123, 1-61.

Maiman, D. J., Kumaresan, S., Yoganandan, N., and Pintar, F. A. (1999) Biomechanical effect of anterior cervical spine fusion on adjacent segments. Biomedical Materials and Engineering 9, 2738. 
Matsunaga, S., Kabayama, S., Yamamoto, T., Yone, K., Sakou, T., and Nakanishi, K. (1999) Strain on intervertebral discs after anterior cervical decompression and fusion. Spine 24, 670675.

Maurel, N., Lavaste, F., and Skalli, W. (1997) A three-dimensional parameterized finite element model of the lower cervical spine. Study of the influence of the posterior articular facets. Journal of Biomechanics 30, 921-931.

Mercer, S. and Bogduk, N. (1999) The ligaments and annulus fibrosus of human adult cervical intervertebral discs. Spine 24, 619-626.

Merrill, T., Goldsmith, W., and Deng, Y. C. (1984) Three-dimensional response of a lumped parameter head-neck model due to impact and impulsive loading. Journal of Biomechanics 17, 81-95.

Milne, N. (1993) Composite motion in cervical disc segments. Clinical Biomechanics 8, 193202.

Milne, N. (1991) The role of zygapophysial joint orientation and uncinate processes in controlling motion in the cervical spine. Journal of Anatomy 178, 189-201.

Moroney, S. P., Schultz, A. B., Miller, J. A., and Andersson, G. B. (1988) Load-displacement properties of lower cervical spine motion segments. Journal of Biomechanics 21, 769-779.

Neptune, R. R. and Kautz, S. A. (2000) Knee joint loading in forward versus backward pedaling: implications for rehabilitation strategies. Clinical Biomechanics 15, 528-535.

Neptune, R. R., Wright, I. C., and van den Bogert, A. J. (2000) The influence of orthotic devices and vastus medialis strength and timing on patellofemoral loads during running. Clinical Biomechanics 15, 611-618.

$\mathrm{Ng}, \mathrm{H}$. W. and Teo, E. C. (2001) Non-linear finite-element analysis of the lower cervical spine (C4-C6) under axial loading. Journal of Spinal Disorders 14, 201-210.

Ng, H. W., Teo, E. C., Lee, K. K., and Qiu, T. X. (2003) Finite element analysis of cervical spinal instability under physiologic loading. Journal of Spinal Disorders 16, 55-65.

Nigg, B. M., and Herzog, W. (1999) Biomechanics of the Musculo-skeletal System. Wiley, Chichester; New York. 
Niosi, C. A. and Oxland, T. R. (2004) Degenerative mechanics of the lumbar spine. Spine Journal 4, 202S-208S.

Nowitzke, A., Westaway, M., and Bogduk, N. (1994) Cervical zygapophyseal joints: geometrical parameters and relationship to cervical kinematics. Clinical Biomechanics 9, 342-348.

Onan, O. A., Heggeness, M. H., and Hipp, J. A. (1998a) A motion analysis of the cervical facet joint. Spine 23, 430-439.

Onan, O. A., Hipp, J. A., and Heggeness, M. H. (1998b) Use of computed tomography image processing for mapping of human cervical facet surface geometry. Medical Engineering and Physics 20, 77-81.

Panjabi, M. M. (1998) Cervical spine models for biomechanical research. Spine 23, 2684-2700.

Panjabi, M. M., Crisco, J. J., Vasavada, A., Oda, T., Cholewicki, J., Nibu, K., and Shin, E. (2001) Mechanical properties of the human cervical spine as shown by three-dimensional loaddisplacement curves. Spine 26, 2692-2700.

Panjabi, M. M., Oxland, T., Takata, K., Goel, V., Duranceau, J., and Krag, M. (1993) Articular facets of the human spine. Quantitative three-dimensional anatomy. Spine 18, 1298-1310.

Panjabi, M. M., Oxland, T. R., and Parks, E. H. (1991) Quantitative anatomy of cervical spine ligaments. Part II. Middle and lower cervical spine. Journal of Spinal Disorders 4, 277-285.

Panjabi, M. M., Summers, D. J., Pelker, R. R., Videman, T., Friedlaender, G. E., and Southwick, W. O. (1986) Three-dimensional load-displacement curves due to forces on the cervical spine. Journal of Orthopaedic Research 4, 152-161.

Penning, L. and Wilmink, J. T. (1987) Rotation of the cervical spine. A CT study in normal subjects. Spine 12, 732-738.

Piazza, S. J. and Delp, S. L. (2001) Three-dimensional dynamic simulation of total knee replacement motion during a step-up task. Journal of Biomechanical Engineering 123, 599-606.

Porcher Nedel, L. and Thalmann, D. (2000) Anatomic modeling of deformable human bodies. The Visual Computer 16, 306-321.

Pospiech, J., Stolke, D., Wilke, H. J., and Claes, L. E. (1999) Intradiscal pressure recordings in the cervical spine. Neurosurgery 44, 379-384. 
Schwab, J. S. (2003) Motion compensation associated with single-level cervical fusion: a multilevel in-vitro study. Master Thesis, The University of Tennessee Health Science Center.

Sekhon, L. H. (2003) Cervical arthroplasty in the management of spondylotic myelopathy. Journal of Spinal Disorders and Technique 16, 307-313.

Sengupta, D. K. (2004) Dynamic stabilization devices in the treatment of low back pain. Orthopedic Clinics of North America. 35, 43-56.

Shea, M., Edwards, W. T., White, A. A., and Hayes, W. C. (1991) Variations of stiffness and strength along the human cervical spine. Journal of Biomechanics 24, 95-107.

Singh, K., Vaccaro, A. R., and Albert, T. J. (2004) Assessing the potential impact of total disc arthroplasty on surgeon practice patterns in North America. Spine Journal 4, S195-S201.

Smith, H. E., Wimberley, D. W., and Vaccaro, A. R. (2004) Cervical arthroplasty: material properties. Neurosurgical Focus 17(3):E1, 15-21.

Szpalski, M., Gunzburg, R., and Mayer, M. (2002) Spine arthroplasty: a historical review. European Spine Journal 11 Suppl 2, S65-S84.

Teo, E. C. and Ng, H. W. (2001a) Evaluation of the role of ligaments, facets and disc nucleus in lower cervical spine under compression and sagittal moments using finite element method. Medical Engineering and Physics 23, 155-164.

Teo, E. C. and Ng, H. W. (2001b) First cervical vertebra (atlas) fracture mechanism studies using finite element method. Journal of Biomechanics 34, 13-21.

Traynelis, V. C. (2004) The Prestige cervical disc replacement. Spine Journal 4, S310-S314.

Treppo, S., Koepp, H., Quan, E. C., Cole, A. A., Kuettner, K. E., and Grodzinsky, A. J. (2000) Comparison of biomechanical and biochemical properties of cartilage from human knee and ankle pairs. Journal of Orthopaedic Research 18, 739-748.

van der Horst, M. J. (2002) Human head neck response in frontal, lateral and rear end impact loading: modelling and validation. PhD. Thesis, Technical University of Eindhoven.

Vasavada, A. N., Li, S., and Delp, S. L. (1998) Influence of muscle morphometry and moment arms on the moment-generating capacity of human neck muscles. Spine 23, 412-422. 
Voo, L. M., Kumaresan, S., Yoganandan, N., Pintar, F. A., and Cusick, J. F. (1997) Finite element analysis of cervical facetectomy. Spine 22, 964-969.

White, A. A. and Panjabi, M. M. (1990) Clinical Biomechanics of the Spine. J.B. Lippincott Co., Philadelphia.

Wigfield, C., Gill, S., Nelson, R., Langdon, I., Metcalf, N., and Robertson, J. (2002a) Influence of an artificial cervical joint compared with fusion on adjacent-level motion in the treatment of degenerative cervical disc disease. Journal of Neurosurgery 96, 17-21.

Wigfield, C. C., Gill, S. S., Nelson, R. J., Metcalf, N. H., and Robertson, J. T. (2002b) The new Frenchay artificial cervical joint: results from a two-year pilot study. Spine 27, 2446-2452.

Yoganandan, N., Kumaresan, S., and Pintar, F. A. (2000) Geometric and mechanical properties of human cervical spine ligaments. Journal of Biomechanical Engineering. 122, 623-629.

Yoganandan, N., Kumaresan, S., and Pintar, F. A. (2001) Biomechanics of the cervical spine Part 2. Cervical spine soft tissue responses and biomechanical modeling. Clinical Biomechanics $16,1-27$.

Yoganandan, N., Kumaresan, S. C., Voo, L., Pinter, F. A., and Larson, S. J. (1996) Finite element modeling of the C4-C6 cervical spine unit. Medical Engineering and Physics 18, 569574.

Yoganandan, N., Pintar, F., Butler, J., Reinartz, J., Sances, A., and Larson, S. J. (1989) Dynamic response of human cervical spine ligaments. Spine 14, 1102-1110.

Yoganandan, N., Pintar, F. A., Sances, A., and Maiman, D. J. (1991) Strength and motion analysis of the human head-neck complex. Journal of Spinal Disorders 4, 73-85. 


\section{Vita}

Hyung Soo Ahn was born in South Korea in 1966. He attended various schools in South

Korea. While in South Korea, he entered the medical school of the Kyungpook National

University (Daegu, South Korea) in 1985, and earned his doctor's degree of Medicine in

February 1991. The following March he entered the internship and resident training in the

orthopedic department of Kyungpook National University Hospital. In February 1996, he

graduated from the training and became a specialist in orthopedics. As a fulfillment of duty to

South Korea, he serviced in military hospitals (215 MASH and Jin-Hae Military Hospital) as an

army surgeon (captain) for 3 years. In May 1999, he started work in hospitals (Young-Joo St.

Peter's Hospital and Sung-Joo Se-Gang Hospital) as a physician. In May 2000, he enrolled in

the post-graduate fellowship training for hand surgery in the orthopedic department of

Kyungpook National University Hospital. In March 2001, he started work at the Robotics and

Biomechatronics Laboratory in POSTECH in South Korea as a postdoctoral researcher. In

January 2002, he came to the US and enrolled in the Joint Program of Biomedical Engineering at

the University of Tennessee Health Science Center and the University of Memphis. After

graduation, Hyung Soo Ahn plans to continue pursuing his research interests in the computer

modeling of the musculoskeletal system. 